\title{
Genome sequence of the Brown Norway rat yields insights into mammalian evolution
}

\author{
Rat Genome Sequencing Project Consortium* \\ ${ }^{*}$ Lists of participants and affiliations appear at the end of the paper
}

The laboratory rat (Rattus norvegicus) is an indispensable tool in experimental medicine and drug development, having made inestimable contributions to human health. We report here the genome sequence of the Brown Norway (BN) rat strain. The sequence represents a high-quality 'draft' covering over $90 \%$ of the genome. The BN rat sequence is the third complete mammalian genome to be deciphered, and three-way comparisons with the human and mouse genomes resolve details of mammalian evolution. This first comprehensive analysis includes genes and proteins and their relation to human disease, repeated sequences, comparative genome-wide studies of mammalian orthologous chromosomal regions and rearrangement breakpoints, reconstruction of ancestral karyotypes and the events leading to existing species, rates of variation, and lineage-specific and lineageindependent evolutionary events such as expansion of gene families, orthology relations and protein evolution.

Darwin believed that "natural selection will always act very slowly, often only at long intervals of time". The consequences of evolution over timescales of approximately 1,000 millions of years (Myr) and $75 \mathrm{Myr}$ were investigated in publications comparing the human with invertebrate and mouse genomes, respectively ${ }^{2,3}$. Here we describe changes in mammalian genomes that occurred in a shorter time interval, approximately $12-24 \mathrm{Myr}$ (refs 4, 5) since the common ancestor of rat and mouse.

The comparison of these genomes has produced a number of insights:

- The rat genome (2.75 gigabases, Gb) is smaller than the human (2.9 Gb) but appears larger than the mouse (initially $2.5 \mathrm{~Gb}$ (ref. 3 ) but given as $2.6 \mathrm{~Gb}$ in NCBI build 32, see http://www.ncbi.nlm. nih.gov/genome/seq/NCBIContigInfo.html).

- The rat, mouse and human genomes encode similar numbers of genes. The majority have persisted without deletion or duplication since the last common ancestor. Intronic structures are well conserved.

- Some genes found in rat, but not mouse, arose through expansion of gene families. These include genes producing pheromones, or involved in immunity, chemosensation, detoxification or proteolysis.

- Almost all human genes known to be associated with disease have orthologues in the rat genome but their rates of synonymous substitution are significantly different from the remaining genes.

- About $3 \%$ of the rat genome is in large segmental duplications, a fraction intermediate between mouse (1-2\%) and human (5-6\%). These occur predominantly in pericentromeric regions. Recent expansions of major gene families are due to these genomic duplications.

- The eutherian core of the rat genome-that is, bases that align orthologously to mouse and human-comprises a billion nucleotides ( $\sim 40 \%$ of the euchromatic rat genome) and contains the vast majority of exons and known regulatory elements $(1-2 \%$ of the genome). A portion of this core constituting $5-6 \%$ of the genome appears to be under selective constraint in rodents and primates, while the remainder appears to be evolving neutrally.

- Approximately $30 \%$ of the rat genome aligns only with mouse, a considerable portion of which is rodent-specific repeats. Of the non-aligning portion, at least half is rat-specific repeats.

- More genomic changes occurred in the rodent lineages than the primate: (1) These rodent genomic changes include approximately 250 large rearrangements between a hypothetical murid ancestor and human, approximately 50 from the murid ancestor to rat, and about the same from the murid ancestor to mouse. (2) A threefoldhigher rate of base substitution in neutral DNA is found along the rodent lineage when compared with the human lineage, with the rate on the rat branch $5-10 \%$ higher than along the mouse branch. (3) Microdeletions occur at an approximately twofold-higher rate than microinsertions in both rat and mouse branches.

- A strong correlation exists between local rates of microinsertions and microdeletions, transposable element insertion, and nucleotide substitutions since divergence of rat and mouse, even though these events occurred independently in the two lineages.

\section{Background}

\section{History of the rat}

The rat, hated and loved at once, is both scourge and servant to mankind. The "Devil's Lapdog" is the first sign in the Chinese zodiac and traditionally carries the Hindu god Ganesh ${ }^{6}$. Rats are a reservoir of pathogens, known to carry over 70 diseases. They are involved in the transmission of infectious diseases to man, including cholera, bubonic plague, typhus, leptospirosis, cowpox and hantavirus infections. The rat remains a major pest, contributing to famine with other rodents by eating around one-fifth of the world's food harvest.

Paradoxically, the rat's contribution to human health cannot be overestimated, from testing new drugs, to understanding essential nutrients, to increasing knowledge of the pathobiology of human disease. In many parts of the world the rat remains a source of meat.

The laboratory rat ( $R$. norvegicus) originated in central Asia and its success at spreading throughout the world can be directly attributed to its relationship with humans ${ }^{7}$. J. Berkenhout, in his 1769 treatise Outline of the Natural History of Great Britain, mistakenly took it to be from Norway and used $R$. norvegicus Berkenhout in the first formal Linnaean description of the species. Whereas the black rat (Rattus rattus) was part of the European landscape from at least the third century $A D$ and is the species associated with the spread of bubonic plague, $R$. norvegicus probably originated in northern China and migrated to Europe somewhere 
around the eighteenth century ${ }^{8}$. They may have entered Europe after an earthquake in 1727 by swimming the Volga river.

\section{The rat in research}

$R$. norvegicus was the first mammalian species to be domesticated for scientific research, with work dating to before 1828 (ref. 9). The first recorded breeding colony for rats was established in 1856 (ref. 9). Rat genetics had a surprisingly early start. The first studies by Crampe from 1877 to 1885 focused on the inheritance of coat colour $^{10}$. Following the rediscovery of Mendel's laws at the turn of the century, Bateson used these concepts in 1903 to demonstrate that rat coat colour is a mendelian trait ${ }^{10}$. The first inbred rat strain, PA, was established by King in 1909, the same year that systematic inbreeding began for the mouse ${ }^{10}$. Despite this, the mouse became the dominant model for mammalian geneticists, while the rat became the model of choice for physiologists, nutritionists and other biomedical researchers. Nevertheless, there are over 234 inbred strains of $R$. norvegicus developed by selective breeding, which 'fixes' natural disease alleles in particular strains or colonies ${ }^{11}$.

Over the past century, the role of the rat in medicine has transformed from carrier of contagious diseases to indispensable tool in experimental medicine and drug development. Current examples of use of the rat in human medical research include surgery $^{12}$, transplantation ${ }^{13-15}$, cancer $^{16,17}$, diabetes ${ }^{18,19}$, psychiatric disorders $^{20}$ including behavioural intervention ${ }^{21}$ and addiction ${ }^{22}$, neural regeneration ${ }^{23,24}$, wound ${ }^{25,26}$ and bone healing ${ }^{27}$, space motion sickness ${ }^{28}$, and cardiovascular disease ${ }^{29-31}$. In drug development, the rat is routinely employed both to demonstrate therapeutic efficacy ${ }^{15,32,33}$ and to assess toxicity of novel therapeutic compounds before human clinical trials ${ }^{34-37}$.

\section{The Rat Genome Project}

Over the past decade, investigators and funding agencies have participated in rat genomics to develop valuable resources. Before the launch of the Rat Genome Sequencing Project (RGSP), there was much debate about the overall value of the rat genome sequence and its contribution to the utility of the rat as a model organism. The debate was fuelled by the naive belief that the rat and mouse were so similar morphologically and evolutionarily that the rat sequence would be redundant. Nevertheless, an effort spearheaded by two NIH agencies (NHGRI and NHLBI) culminated in the formation of the RGSP Consortium (RGSPC)

The RGSP was to generate a draft sequence of the rat genome, and, unlike the comparable human and mouse projects, errors would not ultimately be corrected in a finished sequence ${ }^{38}$. Consequently, the draft quality was critical. Although it was expected to have gaps and areas of inaccuracy, the overall sequence quality had to be high enough to support detailed analyses.

The $\mathrm{BN}$ rat was selected as a sequencing target by the research community. An inbred animal (BN/SsNHsd) was obtained by the Medical College of Wisconsin (MCW) from Harlan Sprague Dawley. Microsatellite studies indicated heterozygosity, so over 13 generations of additional inbreeding were performed at the MCW, resulting in BN/SsNHsd/Mcwi animals. Most of the sequence data were from two females, with a small amount of whole genome shotgun (WGS) and flow-sorted Y chromosome sequencing from a male. The $\mathrm{Y}$ chromosome is not included in the current assembly.

A network of centres generated data and resources, led by the Baylor College of Medicine Human Genome Sequencing Center (BCM-HGSC) and including Celera Genomics, the Genome Therapeutics Corporation, the British Columbia Cancer Agency Genome Sciences Centre, The Institute for Genomic Research, the University of Utah, the Medical College of Wisconsin, The Children's Hospital of Oakland Research Institute, and the Max Delbrück Center for Molecular Medicine, Berlin. After assembly of the genome at the
BCM-HGSC, analysis was performed by an international team, representing over 20 groups in six countries and relying largely on gene and protein predictions produced by Ensembl.

\section{Determination of the genome sequence}

\section{Atlas and the 'combined' sequencing strategy}

Despite progress in assembling draft sequences ${ }^{2,3,39-44}$ the question of which method produces the highest-quality products is unresolved. A significant issue is the choice between logistically simpler WGS approaches versus more complex strategies employing bacterial artificial chromosome (BAC) clones ${ }^{45-48}$. In the Public Human Genome Project ${ }^{2}$ a BAC by BAC hierarchical approach was used and provided advantages in assembling difficult parts of the genome. The draft mouse sequence was a pure WGS approach using the ARACHNE assembler ${ }^{3,49,50}$ but underrepresented duplicated regions owing to 'collapses' in the assembly ${ }^{3,51-53}$. This limitation of the mouse draft sequence was tolerable owing to the planned full use of BAC clones in constructing the final finished sequence.

The RGSPC opted to develop a 'combined' approach using both WGS and BAC sequencing (Fig. 1). In the combined approach, WGS data are progressively melded with light sequence coverage of individual BACs (BAC skims) to yield intermediate products called 'enriched BACs' (eBACs). eBACs covering the whole genome are then joined into longer structures (bactigs). Bactigs are joined to form larger structures: superbactigs, then ultrabactigs. During this process other data are introduced, including BAC end sequences, DNA fingerprints and other long-range information (genetic markers, syntenic information), but the process is constrained by eBAC structures.

To execute the combined strategy we developed the Atlas software package $^{54}$ (Fig. 1). The Atlas suite includes a 'BAC-Fisher' component that performs the functions needed to generate eBACs. WGS genome coverage was generated ahead of complete BAC coverage, so a BAC-Fisher web server was established at the BCM-HGSC to enable users to access the combined BAC and WGS reads as each BAC was processed (see Methods for data access). Each eBAC is assembled with high stringency to represent the local sequence accurately, and so provide a valuable intermediate product that assists all users of the genome data. Additional Atlas modules joined eBACs and linked bactigs to give the complete assembly (Fig. 1). Overall, the combined approach takes advantage of the strengths of both previous methods, with few of the disadvantages.

\section{Sequence and genome data}

Over 44 million DNA sequence reads were generated (Table 1; Methods). Following removal of low-quality reads and vector contaminants, 36 million reads were used for Atlas assembly, which retained 34 million reads. This was $7 \times$ sequence coverage with $60 \%$ provided by WGS and $40 \%$ from BACs. Slightly different estimates came from considering the entire 'trimmed' length of the sequence data $(7.3 \times)$, or only the portion of Phred20 quality or higher $(6.9 \times)$.

The sequence data were end-reads from clones either derived directly from the genome (insert sizes of $<10 \mathrm{~kb}, 10 \mathrm{~kb}, 50 \mathrm{~kb}$ and $>150 \mathrm{~kb}$ ) or from small insert plasmids subcloned from BACs. Overall, these provided 42-fold clone coverage, with 32-fold coverage having both paired ends represented. Approximately equal contributions of clone coverage were from the different categories.

Over 21,000 BACs were used for BAC skims (1.6× coverage) with an average sequence depth of $1.8 \times$, giving an overall $2.8 \times$ genomic sequence coverage from BACs. This was slightly more than the most efficient procedure would require $(\sim 1.2 \times$ each $)$, because the genome size was not known at the project start.

Simultaneous with sequencing, 199,782 clones from the CHORI-230 BAC library ${ }^{55}$ were fingerprinted by restriction enzyme 


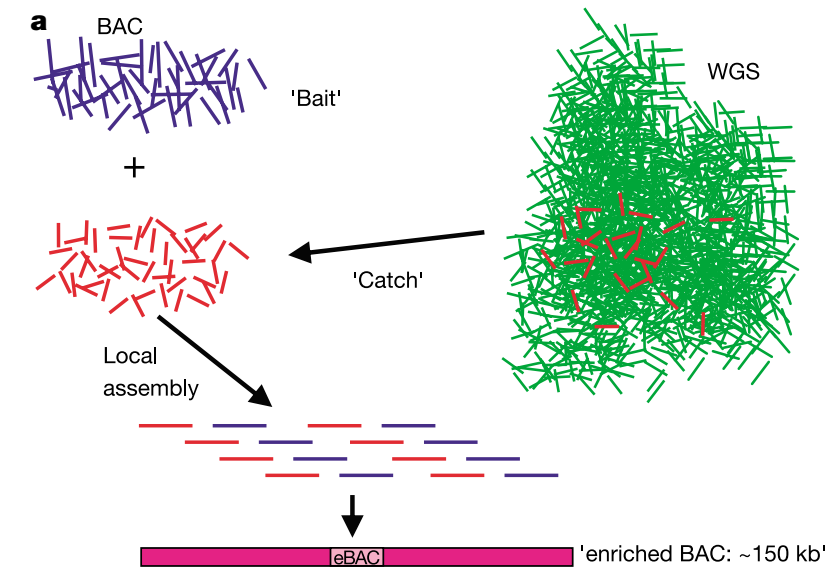

Figure 1 The new 'combined' sequence strategy and Atlas software. a, Formation of 'eBACs'. The RGSP strategy combined the advantages of both BAC and WGS sequence data $^{54}$. Modest sequence coverage ( 1.8 -fold) from a BAC is used as 'bait' to 'catch' WGS reads from the same region of the genome. These reads, and their mate pairs, are assembled using Phrap to form an eBAC. This stringent local assembly retains $95 \%$ of the 'catch'. b. Creation of higher-order structures. Multiple eBACs are assembled into bactigs

digestion, representing 12-fold genomic coverage ${ }^{56}$ (Methods). These were assembled into a 'fingerprint contig (FPC)' map (a contig is a set of overlapping segments of DNA) containing 11,274 FPCs. BAC selection for sequence skimming was based on overlaps between BACs using FPC mapping ${ }^{56}$ (M.K. and C.F., unpublished work), ongoing BAC end sequencing (S.Z., unpublished work), and $\mathrm{BAC}$ sequence skimming ${ }^{57}$. This strategy led to the sequence of a tiling path of BAC clones, covering the whole genome. In addition to the FPC map, a yeast artificial chromosome (YAC)-based physical map was constructed. 5,803 BAC and P1-derived artificial chromosome (PAC) clones from RPCI-32 and RPCI-31 libraries ${ }^{55}$, respectively, were anchored to 51,323 YAC clones originating from two tenfold-coverage YAC libraries ${ }^{58,225}$ assembled into 605 contigs $^{56}$. This map was subsequently integrated with the FPC map and the sequence assembly, reducing the total number of map contigs to 376 (minimum length of contig containing the 'typical' nucleotide, $N_{50}=172$ clones, $4.4 \mathrm{Mb} ; 358$ anchored to the sequence assembly; Supplementary Information).

The combined strategy enabled development of resources such as the FPC map, BAC end sequences, and BAC skim sequences in parallel, rather than sequentially. In addition to allowing ongoing

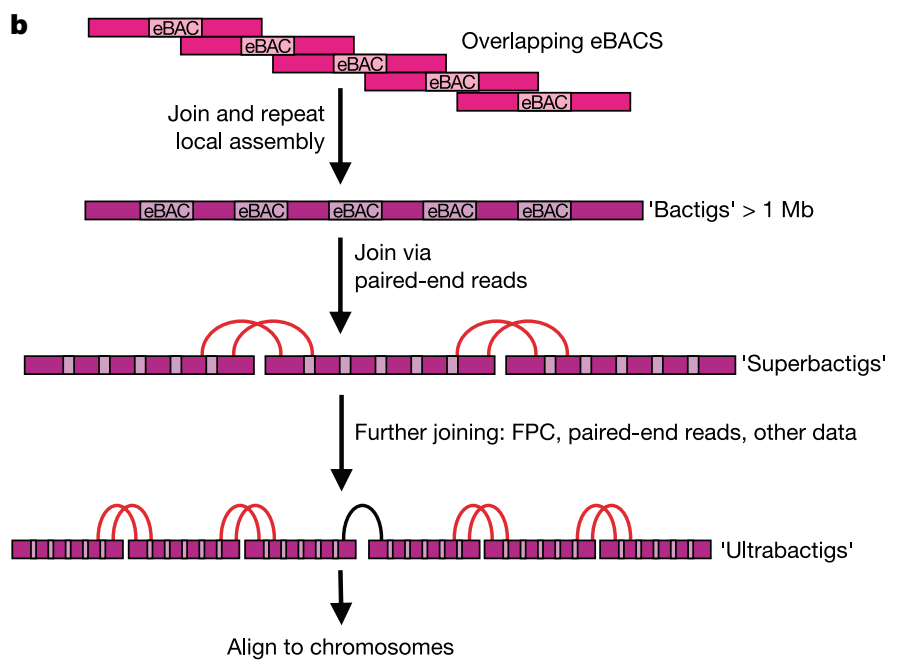

based on sequence overlaps. The bactigs are joined into superbactigs by large clone mate-pair information (at least two links), extended into ultrabactigs using additional information (single links, FPC contigs, synteny, markers), and ultimately aligned to genome mapping data (radiation hybrid and physical maps) to form the complete assembly.

quality checking, this permitted the data-gathering phase of the project to be completed in less than two years.

\section{Atlas assembly}

Statistics for the Rnor3.1 assembly are in Table 2. Contigs within eBACs were ordered and oriented using read-pair information. Read-pair information was also used to add WGS reads to eBACs, even when sequence overlaps could not be reliably detected owing to repeated sequences. BAC skim reads with repeats were included in the assembly of eBACs because they clearly originated within BAC insert sequences. Over 19,000 eBACs were eventually generated.

More than $98 \%$ of eBACs were successfully merged to form bactigs (Fig. 1). Bactigs were subsequently reassembled to process all reads from overlapping BACs simultaneously, and then ordered and oriented with respect to each other using FPC map and BAC end sequence read-pair information. These superbactig and ultrabactig structures (see below) were aligned with chromosomes using external information, such as positions of genetic markers. Ultrabactigs represented the largest sequence units used to build chromosomes.

The current release of the rat genome assembly, version Rnor3.1,

\begin{tabular}{|c|c|c|c|c|c|c|c|c|c|c|}
\hline \multirow[t]{2}{*}{ Insert size ${ }^{\star}(\mathrm{kb})$} & \multirow[t]{2}{*}{ Source or vector } & \multicolumn{4}{|c|}{ Reads (millions) } & \multicolumn{2}{|c|}{ Bases (billions) } & \multicolumn{2}{|c|}{ Sequence coverage $\dagger$} & \multirow[t]{2}{*}{ Clone coverage } \\
\hline & & All§ & Used & Paired & Assembled & Trimmed & $\geq$ Phred20 & Trimmed & $\geq$ Phred20 & \\
\hline $2-4$ & Plasmid & 9.6 & 8.6 & 7.4 & 7.9 & 4.8 & 4.5 & 1.8 & 1.6 & 3.70 \\
\hline $4.5-7.5$ & Plasmid & 4.5 & 4.3 & 3.6 & 3.6 & 2.4 & 2.3 & 0.87 & 0.82 & 2.96 \\
\hline 10 & Plasmid & 8.4 & 7.2 & 6.4 & 6.4 & 4.1 & 3.8 & 1.5 & 1.4 & 11.63 \\
\hline 50 & Plasmid & 1.7 & 1.3 & 1.0 & 1.1 & 0.69 & 0.65 & 0.25 & 0.24 & 9.47 \\
\hline $150-250$ & BAC & 0.32 & 0.31 & 0.26 & 0.26 & 0.18 & 0.16 & 0.07 & 0.06 & 9.26 \\
\hline Total WGS & & 24.5 & 21.7 & 18.7 & 19.2 & 12.1 & 11.3 & 4.4 & 4.1 & 37.0 \\
\hline $2-5$ & BAC skims & 19.6 & 14.6 & 13.2 & 14.5 & 8.0 & 7.7 & 2.9 & 2.8 & $4.8 \|$ \\
\hline Total & & 44.1 & 36.3 & 31.9 & 33.7 & 20.2 & 19.0 & 7.3 & 6.9 & 41.8 \\
\hline
\end{tabular}

* Grouped in ranges of sizes for individual libraries tracked to specific multiples of $0.5 \mathrm{~kb}$.

†Total bases in used reads divided by sampled genome size including all cloned and sequenced euchromatic or heterochromatic regions.

‡Estimated as sum of insert sizes divided by sampled genome size.

§WGS reads available on the NCBI Trace Archive as of 21 March 2003; BAC skim reads attempted at BCM-HGSC as of 12 May 2003 ; BAC end reads obtained directly from TIGR.

$\|$ Refers to coverage from $2-5 \mathrm{~kb}$ subclones from BACs. The BACs that were skimmed amounted to $1.58 \times$ clone coverage. 
Table 2 Statistics of the RGSP draft sequence assembly

\begin{tabular}{|c|c|c|c|c|c|c|c|c|}
\hline \multirow[t]{2}{*}{ Features* } & \multirow[t]{2}{*}{ Number } & \multirow{2}{*}{$\begin{array}{l}\text { N50 length } \\
(\mathrm{kb})\end{array}$} & \multirow{2}{*}{$\begin{array}{l}\text { Bases } \\
(\mathrm{Gb})\end{array}$} & \multirow{2}{*}{$\begin{array}{c}\text { Bases plus gaps } \dagger \\
(\mathrm{Gb})\end{array}$} & \multicolumn{4}{|c|}{ Percentage of genome $\neq$} \\
\hline & & & & & \multicolumn{2}{|c|}{ Sampled (2.78 Gb) } & \multicolumn{2}{|c|}{ Assembled (2.75 Gb) } \\
\hline & & & & & Bases & Bases + gaps & Bases & Bases + gaps \\
\hline Anchored contigs & 127,810 & 38 & 2.476 & 2.481 & 89.1 & 89.2 & 90.0 & 90.2 \\
\hline Anchored superbactig scaffolds & 783 & 5,402 & 2.476 & 2.509 & 89.1 & 90.3 & 90.0 & 91.2 \\
\hline Anchored ultrabactigs & 291 & 18,985 & 2.476 & 2.687 & 89.1 & 96.6 & 90.0 & 97.7 \\
\hline Unanchored superbactigs, main scaffolds & 134 & 1,210 & 0.056 & 0.062 & 2.0 & 2.2 & 2.0 & 2.3 \\
\hline Unanchored ultrabactigs & 128 & 1,529 & 0.056 & 0.069 & 2.0 & 2.5 & 2.0 & 2.5 \\
\hline All superbactigs, main scaffolds & 917 & 5,301 & 2.533 & 2.571 & 91.1 & 92.5 & 92.1 & 93.5 \\
\hline Minor scaffolds & 4,345 & 8 & 0.033 & 0.038 & 1.2 & 1.4 & 1.2 & 1.4 \\
\hline
\end{tabular}

*Anchored sequences are those that can be placed on chromosomes because they contain known markers. The main scaffold for each superbactig is the largest set of contigs (in terms of total contig sequence) that can be ordered and oriented using mate-pair links and ordering of BACs. Scaffolds that cannot be ordered and oriented with respect to the main scaffold are termed minor scaffolds. tAmbiguous bases $(\mathrm{N})$ are counted in the gap sizes, and excluded in the base counts.

$\ddagger$ Computed as bases plus gaps divided by estimated genome size. Sampled genome size is based on oligonucleotide frequency statistics of unassembled WGS reads. Assembled genome size is based on cumulative contig sequence following assembly.

was generated using the data in Table 1. Earlier releases (Rnor2.0/ 2.1, Methods) were used for a substantial part of the annotation and analysis of genes and proteins, whereas the current release provided the genome description. Rnor3.1 has 128,000 contigs, with $N_{50}$ length $38 \mathrm{~kb}$-larger than the expected genomic extent of a mammalian gene. These sequence contigs were linked into 783 superbactigs that were anchored to the radiation hybrid map ${ }^{59}$. These larger units had $N_{50}$ length $5.4 \mathrm{Mb}$. Another 134 smaller superbactigs $\left(N_{50}\right.$ length $\left.1.2 \mathrm{Mb}\right)$ could not be anchored, presumably because they fell into gaps between markers or because they were in repeated regions that could not be unambiguously placed. From placement on the radiation hybrid map, adjacent superbactigs were further linked to maximize continuity of sequence if appropriate read-pair mates existed or FPC suggested links. This reduced linked superbactigs to 419 pieces with 71 singletons. 291 ultrabactigs with $N_{50}$ length of nearly $19 \mathrm{Mb}$ were placed on chromosomes. Orthology information with mouse and human sequences was also used to resolve conflicts and suggest placement of sequence units. Most of the 128 unplaced units were either singletons or small superbactigs that consisted of few clones. Thus, nearly the entire genome was represented in less than 300 large sequence units.

\section{Quality assessment}

Thirteen megabases of high-quality finished rat sequence from BACs were available for comparison with Rnor3.1 (Methods). This analysis showed that the majority of draft bases from within contigs were high quality (1.32 mismatches per $10 \mathrm{~kb})$. This is essentially the accepted accuracy standard for finished sequence (1.0 errors per $10 \mathrm{~kb})^{60}$, so the overwhelming majority of contig bases are highly accurate. The highest frequency of mismatches occurred at the ends of contigs. We calculate the average size of these loweraccuracy regions to be 750 base pairs (bp) and they amount to less than $0.9 \%$ of the genome. These regions arise from misassembly of terminal reads due to repeated sequences.

Few mismatches were found within contigs. Six were found within contigs when compared with the $13 \mathrm{Mb}$ of finished sequence, or one case per $2.2 \mathrm{Mb}$. All were insertions or deletions and may represent polymorphisms. Thus, at the fine structure level, the bulk of sequences that make up contigs is nearly the quality of finished sequence.

We judged accuracy of assembly at the chromosomal level by alignment with linkage maps ${ }^{61}$ and radiation hybrid map ${ }^{59}$ (Fig. 2). Thirteen markers out of 3,824 from the SHRSP $\times$ BN genetic map were placed on different chromosomes in the assembly and in the genetic map. Similarly, of the 20,490 sequence tagged sites placed on both the assembly and radiation hybrid (v3.4) map, 96.9\% had consistent chromosome placement ${ }^{59}$. Initial alignments identified regions of misassembly, and these were corrected, so that in Rnor3.1 the maps are congruent except for possible mismapped markers. The distribution of assembled sequence among the chromo- somes and chromosome sizes in Rnor3.1 are in Supplementary Table SI-2.

\section{Landscape and evolution of the rat genome}

\section{Genome size}

Genomic assemblies are usually smaller than the actual genome size owing to under-representation of sequences affected by cloning bias, and sequencing and assembly difficulties. Simply equating the assembled genome size with the euchromatic, cloneable portion does not take into account heterochromatin that may be included ${ }^{62}$. We therefore estimated both an assembled genome size, scaled by the inverse of the fraction of features (genetic markers, expressed sequence tags (ESTs), and so on) found in the Rnor3.1 assembly, and a cloneable (or sampled) genome size, which was the part of the genome present in the WGS reads before assembly, as measured by analysing the distribution of short oligomers ${ }^{63}$. The former may be an underestimate because non-repetitive, easily assembled regions can be enriched for known features. The latter should be an overestimate because there are likely to be regions (such as repeats) that can be cloned and sequenced, but not assembled.

For the rat genome, the assembled and cloneable genome sizes are very close. Considering the fraction of the marker set successfully mapped to Rnor3.1 (92\%), or the fraction of sequence finished outside the BCM-HGSC (to reduce bias) present in Rnor3.1 (91\%), together with the assembled bases in main scaffolds $(2.533 \mathrm{~Gb}$, Table 2), we suggest a genome size of $2.75 \mathrm{~Gb}$. Alternatively, analysis of the WGS oligomers of length 24 to 32 predicted a genome size of between 2.76 and 2.81 billion bases. We have used the more conservative value of $2.75 \mathrm{~Gb}$ for the rat genome size, but this is

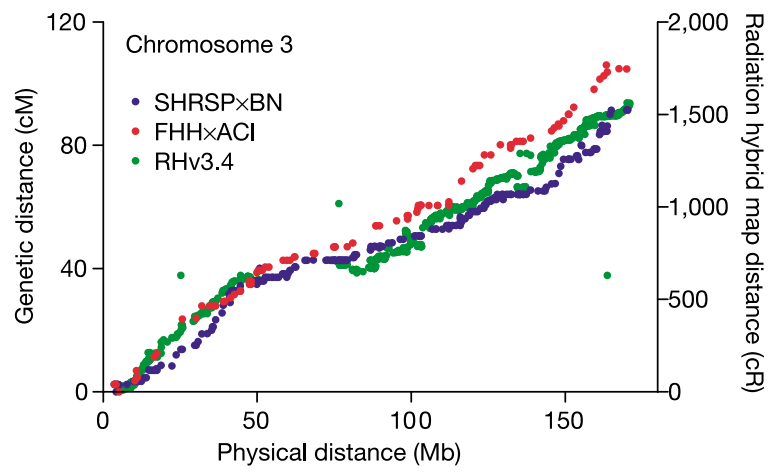

Figure 2 Map correspondence. Correspondence between positions of markers on two genetic maps of the rat (SHRSP $\times \mathrm{BN}$ intercross and $\mathrm{FHH} \times \mathrm{ACl}$ intercross $\left.{ }^{61}\right)$, on the rat radiation hybrid map ${ }^{59}$, and their position on the rat genome assembly (Rnor3.1). 
still considerably higher $(150 \mathrm{Mb})$ than the $2.6 \mathrm{~Gb}$ currently reported for the mouse draft genome sequence. A fraction of the size differences in these rodent genomes results from the different repeat content (see below); however, it is also recognized that segmental duplications may be under-represented in the mouse WGS draft sequence for technical reasons ${ }^{3,51}$.

\section{Telomeres, centromeres and mitochondrial sequence}

The rat has both metacentric and telocentric chromosomes, in contrast to the wholly telocentric mouse chromosomes. As expected from previous draft sequences, the rat draft does not contain complete telomeres or centromeres. Their physical location relative to the rat draft sequence can however be approximated; the centromeres of the telocentric rat chromosomes (2, 4-10 and X) must be positioned before nucleotide 1 of these assemblies, and those for the remaining chromosomes are estimated as indicated in Fig. 3. Several of these putative centromere positions coincide with both segmental duplication blocks (see below) and classical satellite clusters, consistent with enrichment of both of these sequence features in rat pericentromeric DNA. Human subtelomere regions are characterized by both an abundance of segmentally duplicated DNA and an enrichment of internal (TTAGGG) ${ }_{n}$-like sequence islands ${ }^{64}$. Approximately one-third of the euchromatic rat subtelomeric regions are similarly enriched, suggesting that Rnor3.1 might extend very close to the chromosome ends.

Fragments of the rat mitochondrial genome were also propagated within the WGS libraries and subsequently sequenced, allowing the assembly of the complete 16,313 bp mitochondrial genome (Supplementary Information). Comparison with existing mitochondrial sequences in the public databases revealed variable positions totalling $95 \mathrm{bp}(0.6 \%)$ between this strain and the wild brown rat. Considerably more variation $(2.2 \%)$ was found when compared with the Wistar strain: 357 bp differences over the whole genome, including 78 positions that are conserved in the other mammalian sequences. Such variation has also been reported in mouse mitochondrial sequences and attributed to errors in previously

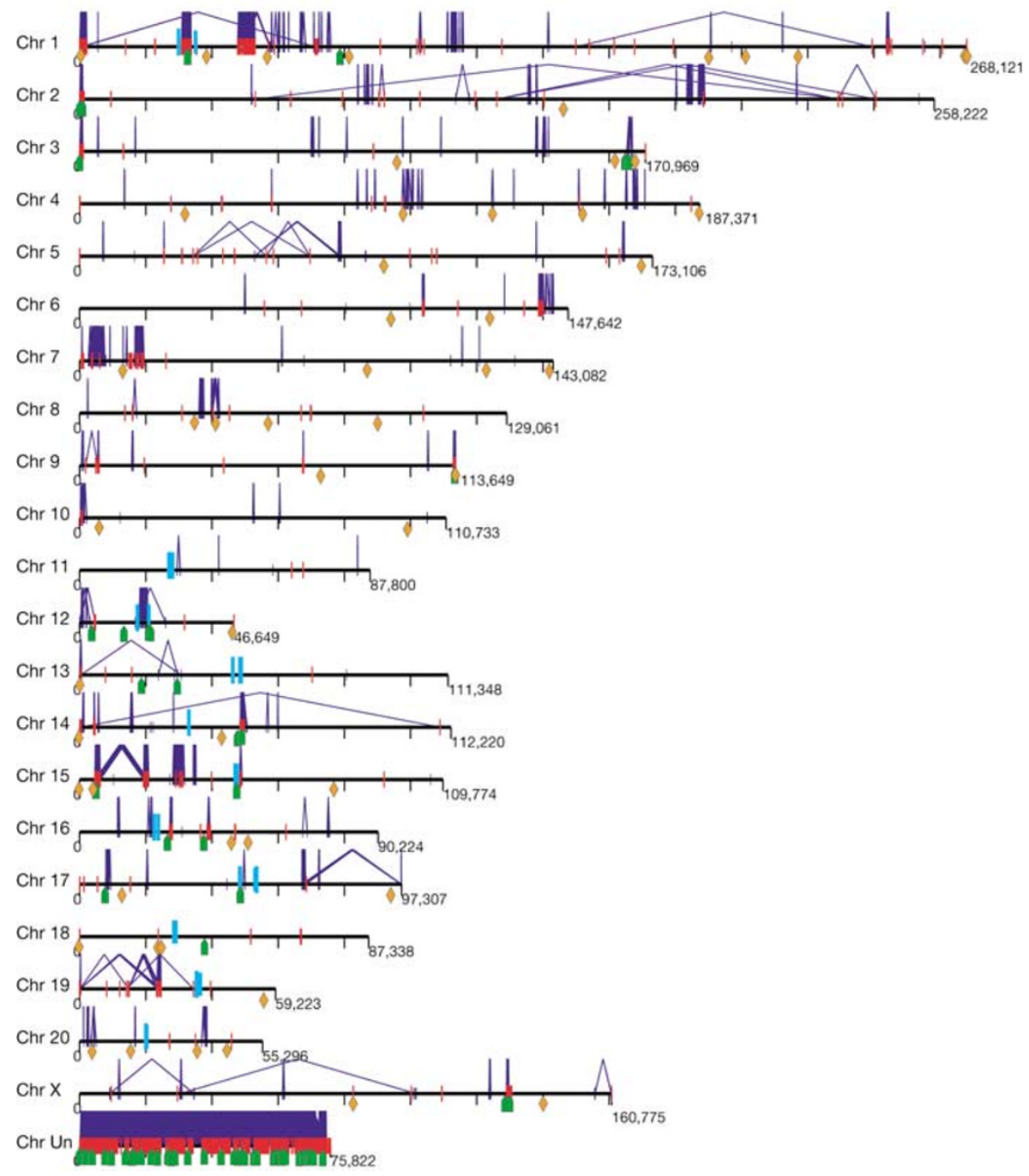

Figure 3 Distribution of segmental duplications in the rat genome. Interchromosomal duplications (red) and intrachromosomal duplications (blue) are depicted for all duplications with $\geq 90 \%$ sequence identity and $\geq 20 \mathrm{~kb}$ length. The intrachromosomal duplications are drawn with connecting blue line segments; those with no apparent connectors are local duplications (spaced below the figure resolution limit). $p$ arms are on the left and the $q$ arms on the right. Chromosomes 2, 4-10, and X are telocentric; the assemblies begin with pericentric sequences of the $q$ arms, and no centromeres are indicated. For the remaining chromosomes, the approximate centromere positions were estimated from the most proximal STS/gene marker to the $p$ and $q$ arm as determined by fluorescent in situ hybridization (FISH) (cyan vertical lines; no chromosome 3 data). The 'Chr Un' sequence consists of contigs not incorporated into any chromosomes. Green arrows indicate $1 \mathrm{Mb}$ intervals with more than tenfold enrichment of classic rat satellite repeats within the assembly. Orange diamonds indicate $1 \mathrm{Mb}$ intervals with more than tenfold enrichment of internal (TTAGGG) ${ }_{n}$-like sequences. For more detail see http:// ratparalogy.cwru.edu. 
sequenced genomes ${ }^{65}$. The current sequence is very accurate, and we therefore favour the $\mathrm{BN}$ sequence as a reference for the rat mitochondrial genome.

\section{Orthologous chromosomal segments and large-scale rearrangements}

Multi-megabase segments of the chromosomes of the primaterodent ancestor have been passed on to human and murid rodent descendants with minimal rearrangements of gene order ${ }^{66-68}$. These intact regions, which are bounded by the breaks that occurred during ancient large-scale chromosomal rearrangements, are referred to as orthologous chromosomal segments. The same phenomenon has occurred in the descent of the rat and mouse from the genome of their common murid ancestor, and we were able to use the human genome, and in some cases other outgroup data, to tentatively reconstruct the sequence of many of these rearrangements in these lineages. To visualize the extent of orthologous chromosomal segments, each genome was 'painted' with the orthologous segments of the other two species (Fig. 4) using the Virtual Genome Painting method (M.L.G.-G. et al., unpublished work; http://www.genboree.org). Inspection shows the interleaving of events that both preceded and occurred subsequently to the ratmouse divergence.

Comparing the three species at $1 \mathrm{Mb}$ resolution, BLASTZ ${ }^{69}$, PatternHunter/Grimm-Synteny ${ }^{70,71}$, Pash $^{72}$, and associated merging algorithms ${ }^{66,72,73}$ produce virtually indistinguishable sets of orthologous chromosomal segments. PatternHunter and the GRIMMSynteny algorithm ${ }^{73}$ detect 278 orthologous segments between human and rat, and 280 between human and mouse. The mouserat comparison reveals a smaller number of segments (105) of larger average size. The larger number of breaks in orthologous segments between the human to the rodent pair is expected, because of the
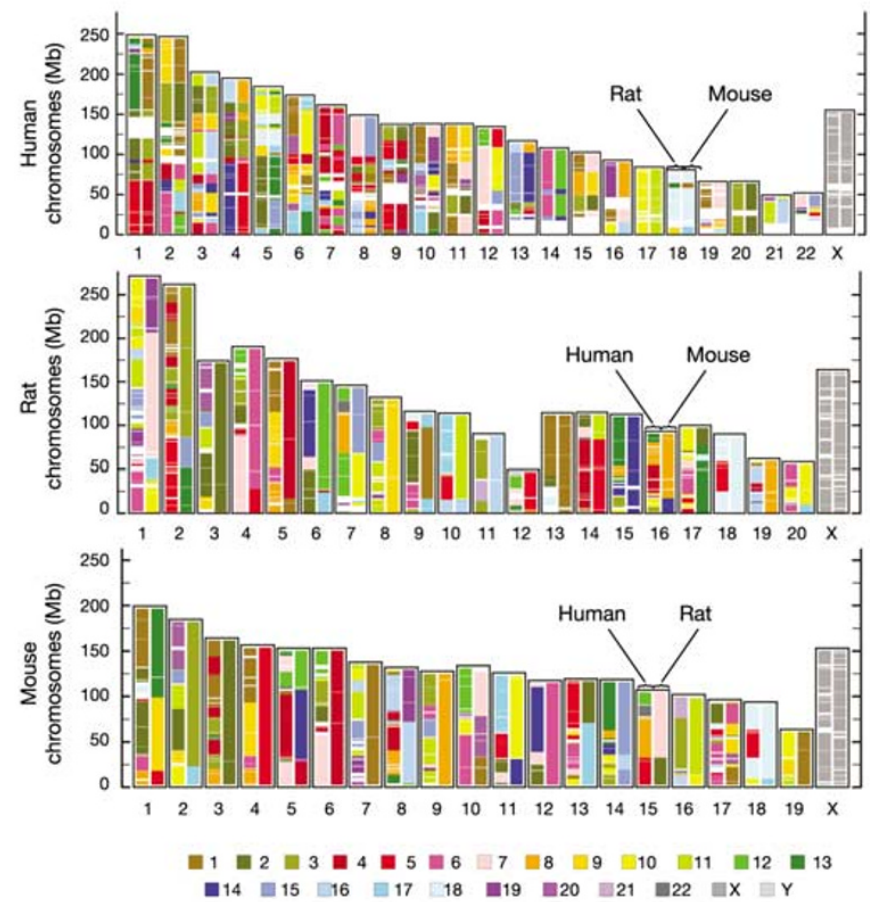

Figure 4 Map of conserved synteny between the human, mouse and rat genomes. For each species, each chromosome ( $x$ axis) is a two-column boxed pane ( $p$ arm at the bottom) coloured according to conserved synteny to chromosomes of the other two species. The same chromosome colour code is used for all species (indicated below). For example, the first $30 \mathrm{Mb}$ of mouse chromosome 15 is shown to be similar to part of human chromosome 5 (by the red in left column) and part of rat chromosome 2 (by the olive in right column). An interactive version is accessible (http://www.genboree.org). latter's closer evolutionary relationship.

Understanding the number and timing of rearrangement events that have occurred in each of the three individual lineages (see tree in Fig. 5a) since the common primate-rodent ancestor required a more detailed analysis. We initially focused on the X chromosome, because rearrangements between the $\mathrm{X}$ and the autosomes are rare ${ }^{74}$ and its history is somewhat easier to trace completely. The $\mathrm{X}$ chromosome consists of 16 human-mouse-rat orthologous segments of at least $300 \mathrm{~kb}$ in size ${ }^{73}$ (Fig. 6a). In the most parsimonious scenario (found with MGR and GRIMM ${ }^{75}$ ), these were created by 15 inversions in the descent from the primate-rodent ancestor (Fig. 6b). Outgroup data from cat, $\operatorname{cow}^{76}$ and $\operatorname{dog}^{77}$ resolved the timing of these rearrangements more precisely. Most of these events occurred in the rodent lineage: five (or four) before the divergence of rat and mouse, five in the rat lineage, and five in the mouse lineage. At most one rearrangement occurred in the human lineage since divergence from the common ancestor with rodents. The timing of this one event was ambiguous, owing to the limited resolution of the outgroup data. Even given this uncertainty, it is clear that the large-scale architecture of the $\mathrm{X}$ chromosome in humans is largely unchanged since the primate-rodent ancestor ${ }^{73}$, whereas there has been considerable activity in the rodents. The assignment of the accelerated activity to the rodent branch, following the primate-rodent divergence, is consistent with previous studies at significantly lower resolution (these showed complete conservation of marker order between the $\mathrm{X}$ chromosomes of human and $\mathrm{cat}^{78}$, human and $\operatorname{dog}^{77}$, and human and lemur ${ }^{79}$, as well as similar karyotypes of the $\mathrm{X}$ chromosomes in human, chimpanzees, gorillas and orangutans ${ }^{80}$ ).

Large-scale reconstruction of the entire ancestral murid genome suggests that it retained many previously postulated chromosome associations of the placental ancestor ${ }^{81,82}$. The most parsimonious scenario we found requires a total of 353 rearrangements: 247 between the murid ancestor and human, 50 from the murid ancestor to mouse and 56 from the murid ancestor to rat. A recent study $^{82}$ implies that most of the 247 rearrangements between the murid ancestor and human occurred on the evolutionary subpath from the squirrel-mouse-rat ancestor to the murid ancestor. Our

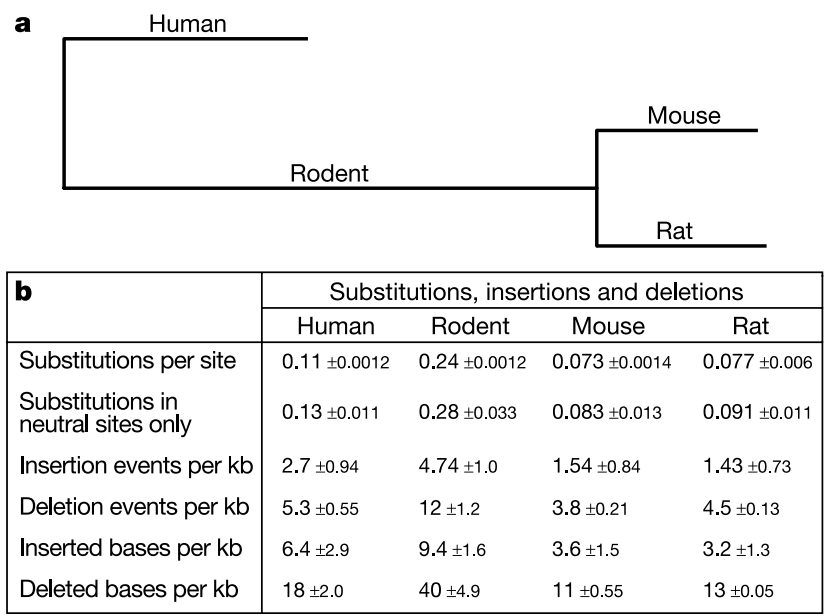

Figure 5 Substitutions and microindels (1-10 bp) in the evolution of the human, mouse and rat genomes. $\mathbf{a}$, The lengths of the labelled branches in the tree are proportional to the number of substitutions per site inferred using the REV mode ${ }^{222}$ from all sites with aligned bases in all three genomes. $\mathbf{b}$, The table shows the midpoint and variation in these branch-length estimates when estimated from different sequence alignment programs and different neutral sites, including sites from ancestral repeats ${ }^{3}$, fourfold degenerate sites in codons, and rodent-specific sites ('in neutral sites only' row; Supplementary Information). Other rows give midpoints and variation for micro-indels on each branch of the tree in $\mathbf{a}$ 
analyses confirm that the rate of rearrangements in murid rodents is much higher than in the human lineage ${ }^{73}$.

\section{Segmental duplications}

Segmental duplications are defined here as regions of the genome that are repeated over at least $5 \mathrm{~kb}$ of length and $>90 \%$ identity. The rat has approximately $2.9 \%$ of its bases in these duplicated regions (Fig. 3), whereas the human genome has $5-6 \%{ }^{83}$. In contrast to the greater rate of large-scale rearrangement, the mouse genome shows substantially fewer of these events ${ }^{3}$, with only $1.0-2.0 \%{ }^{51}$ of its sequenced bases in duplicated regions. These duplicated structures are particularly challenging to assemble, and we attribute at least some of the mouse-rat differences to the BAC-based approach we used for Rnor3.1, compared with the WGS mouse approach. The vast majority of these sequences $(73$ of $82 \mathrm{Mb})$ were regions with $<99.5 \%$ identity and thus were not simply overlapping sequences that had not been joined by the assembly program Phrap. The 'unplaced' chromosome in Rnor3.1 showed a marked enrichment for blocks of segmental duplication (nearly $44 \%$ of the total), which indicates problems with anchoring these elements to the genome.

Intrachromosomal duplications are represented at a three-to-one excess when compared with interchromosomal duplications, and are significantly enriched near the telomeres and in centromeric regions (Fig. 3). The pericentromeric accumulation of segmental duplications in the rat is reminiscent of that observed in human and mouse $^{83-86}$, and seems to be a general property of mammalian chromosome architecture.

We observed considerable clustering of duplications ${ }^{87}$, including 41 discrete genomic regions larger than $1 \mathrm{Mb}$ in size in which duplications appear to be organized into groups with $<100 \mathrm{~kb}$ between duplicated segments. For many of these clusters, the underlying sequence alignments showed a wide range in the degree of sequence identity, suggesting that these areas have been subject to duplication events more or less continuously over millions of years. In contrast, an analysis of the evolutionary distance between all duplicated regions showed an unusual bimodal distribution, particularly for intrachromosomal segmental duplications. Two peaks were observed at 0.045 substitutions per site and 0.075 substitutions per site. Given that the rat genome has accumulated $8-10 \%$ substitutions (see below) since the speciation from mouse 12$24 \mathrm{Myr}$ ago, this bimodal distribution may correspond to bursts of segmental duplication that occurred approximately 5 and $8 \mathrm{Myr}$ ago, respectively.

The segmental duplications in the rat genome were of considerable interest because they represent an important mechanism for the generation of new genes. We found that 63 NCBI reference sequence $^{88}$ (RefSeq; see http://www.ncbi.nih.gov/RefSeq/) genes were located completely or partially within rat duplicated regions, out of a genome total of 4,532 rat RefSeq genes. As discussed below, many of these genes are present in multiple copies and belong to gene familes that have been recently duplicated and contribute to distinctive elements of rat biology.

\section{Gains and losses of DNA}

In addition to large rearrangements and segmental duplications, genome architecture is strongly influenced by insertion and deletion events that add and remove DNA over evolutionary time. To characterize the origins and losses of sequence elements in the human, mouse and rat genomes, we categorized all the nucleotides in each of the three genomes, using our alignment data and

\section{a Synteny blocks}

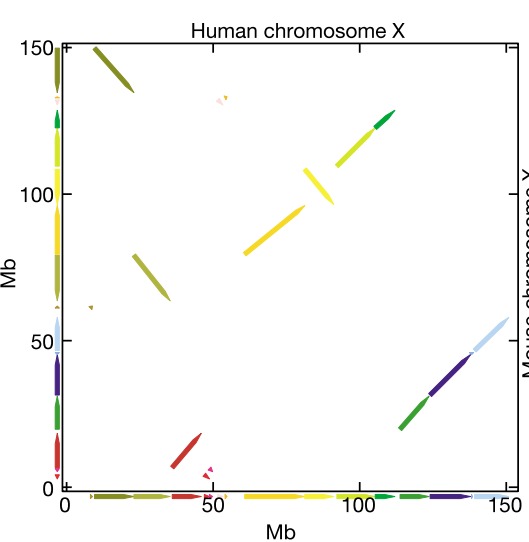

$\mathrm{Mb}$

b A most parsimonious scenario
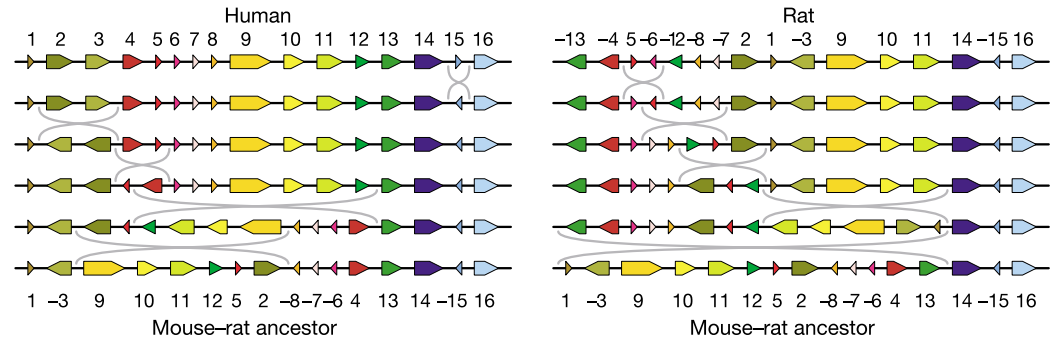

Human chromosome $X$

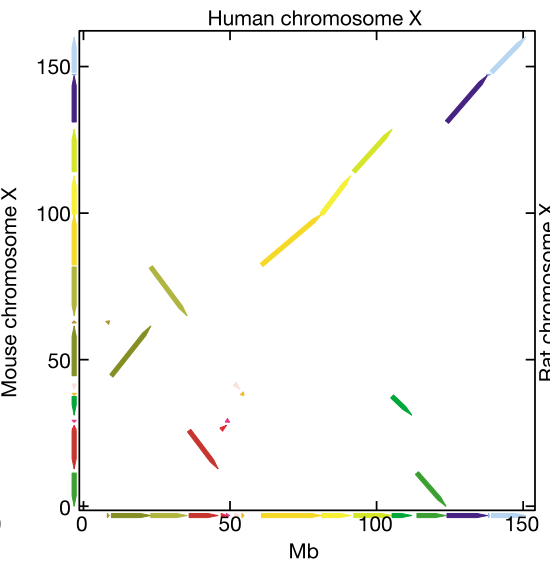

$\mathrm{Mb}$ with 15 events are achievable and all have the same median ancestor (located at the last common mouse-rat ancestor). Shown is a possible (not unique) most parsimonious inversion scenario from each species to that ancestor. We note that the last common ancestor of human, mouse and rat should be on the evolutionary path between this median ancestor and human.

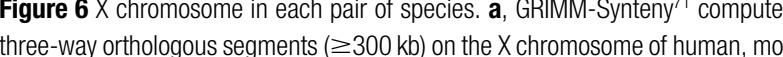
rat, shown for each pair of species, using consistent colours. b. The arrangement (orde and orientation) of the 16 blocks implies that at least 15 rearrangement events occurred during X chromosome evolution of these species. The program MGR (http:// www.cs.ucsd.edu/groups/bioinformatics/MGR/) determined that evolutionary scenarios
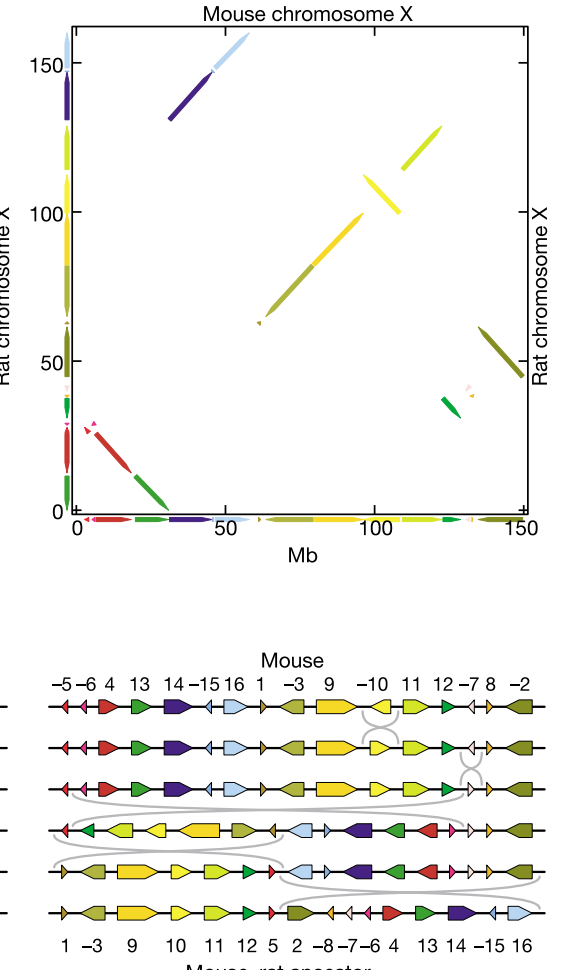

Mouse-rat ancestor 
RepeatMasker annotations of the insertions of repetitive elements (Fig. 7). The rodent repeat database used by RepeatMasker was greatly expanded by analysing the rat and mouse genomes ${ }^{89}$, but it is clear that not all repeats are being recognized, especially the older ones. Thus, these estimates of the amount of rodent repeats represent lower bounds.

About a billion nucleotides (39\% of the euchromatic rat genome) align in all three species, constituting an 'ancestral core' that is retained in these genomes. This ancestral core contains $94-95 \%$ of the known coding exons and regulatory regions. Comparisons between the human and mouse genomes, using transposon relics retained in both species ('mammalian ancestral repeats') to model neutral evolution, have been used to estimate the fraction of the human genome that is accumulating substitutions more slowly than the neutral rate in both lineages since their divergence, and hence may be under some level of purifying selection ${ }^{3}$. Depending on details of methodology, such estimates have ranged between about $4 \%$ and $7 \%{ }^{3,90,91}$. The levels of three-way conservation observed here between the human, mouse and rat genomes in the ancestral core lend further support to these earlier estimates, giving values in the range of 5-6\% when measured by two quite different methods (see Methods and ref. 92). In this constrained fraction, non-coding regions outnumber coding regions regardless of the strength of constraint $^{92}$, an observation that supports recent comparative

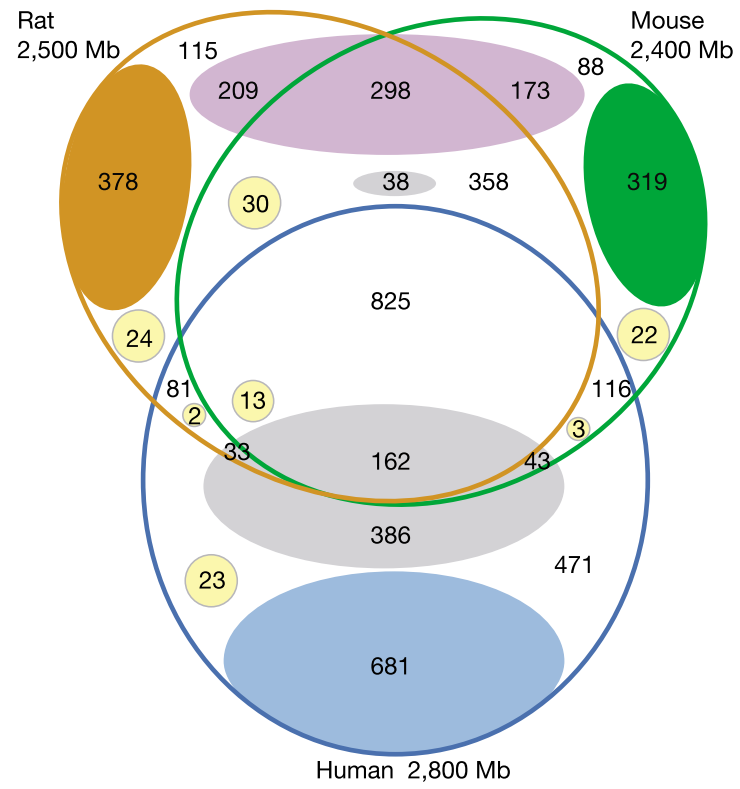

\begin{tabular}{|llll|}
\hline $\begin{array}{l}\text { Genomic } \\
\text { DNA }\end{array}$ & Rat & Mouse & Human \\
$\begin{array}{l}\text { Repetitive } \\
\text { DNA }\end{array}$ & $\begin{array}{l}\text { Ancestral to } \\
\text { human-mouse-rat } \\
\text { Ancestral to } \\
\text { mouse-rat }\end{array}$ & Rat-specific & Primate-specific \\
& $\begin{array}{l}\text { Mouse- } \\
\text { specific }\end{array}$ & Simple \\
\hline
\end{tabular}

Figure 7 Aligning portions and origins of sequences in rat, mouse and human genomes. Each outlined ellipse is a genome, and the overlapping areas indicate the amount of sequence that aligns in all three species (rat, mouse and human) or in only two species. Non-overlapping regions represent sequence that does not align. Types of repeats classified by ancestry: those that predate the human-rodent divergence (grey), those that arose on the rodent lineage before the rat-mouse divergence (lavender), species-specific (orange for rat, green for mouse, blue for human) and simple (yellow), placed to illustrate the approximate amount of each type in each alignment category. Uncoloured areas are non-repetitive DNA-the bulk is assumed to be ancestral to the human-rodent divergence. Numbers of nucleotides (in Mb) are given for each sector (type of sequence and alignment category). Detailed results are tabulated (Supplementary Table SI-1). analyses limited to subsets of the genome $\mathrm{e}^{93,94}$. The preponderance of non-coding elements in the most constrained fraction of the genome underscores the likelihood that they play critical roles in mammalian biology.

About $700 \mathrm{Mb}(28 \%)$ of the rat euchromatic genome aligns only with the mouse. At least $40 \%$ of this comprises of rodent-specific repeats inserted on the branch from the primate-rodent ancestor to the murid ancestor, and some of the remainder can be recognized as mammalian ancestral repeats whose orthologues were deleted in the human lineage (Fig. 7). Another part is likely to consist of singlecopy ancestral DNA deleted in the human lineage but retained in rodents. Although this $700 \mathrm{Mb}$ of rodent-specific DNA is primarily neutral, it may also contain some functional elements lost in the human lineage in addition to sequences representing gains of rodent-specific functions, including some coding exons ${ }^{95}$.

The remainder of the euchromatic rat genome (726 Mb, 29\%) aligns with neither mouse nor human (Fig. 7). At least half of this (15\% of the rat genome) consists of rat-specific repeats, and another large fraction ( $8 \%$ of the rat genome) consists of rodent-specific repeats whose orthologues are deleted in the mouse.

\section{Substitution rates}

The alignment data allow relatively precise estimates of the rates of neutral substitutions and microindel events ( $\leq 10 \mathrm{bp})$. Both synonymous fourfold degenerate ('4D') sites in protein-coding regions and sites in mammalian ancestral repeats were used in this analysis, as in previous studies comparing human and mouse $\mathrm{e}^{3,96}$. We additionally used a class of primarily neutral sites whose identification is made uniquely possible by the addition of the rat genome sequence: namely, the rodent-specific sites discussed above, identified by their failure to align to human sequence.

Our estimates for the neutral substitution level between the two rodents range from 0.15 to 0.20 substitutions per site, while estimates for the entire tree of human, mouse and rat range from 0.52 to 0.65 substitutions per site (Fig. 5). This difference was predictable because of the evolutionary closeness of the two rodents. For all classes of neutral sites analysed, however, the branch connecting the rat to the common rodent ancestor is $5-10 \%$ longer than the mouse branch (Fig. 5a). Thus, for as yet unknown reasons, the rat lineage has accumulated substantially more point substitutions than the mouse lineage since their last common ancestor.

We also analysed four-way alignments including sequence from orthologous ancestral repeats in human, mouse and rat, along with the repeat consensus sequences, which approximate the sequence of the progenitor of the corresponding repeat family (Methods). These alignments allow us to distinguish substitutions on the branch from the primate-rodent ancestor to the rodent ancestor from substitutions on the branch descending to human ${ }^{77}$. This revealed an overall speed-up in rodent substitution rates relative to human of about three-to-one, larger than estimated previously ${ }^{3}$, but consistent with other more recent studies which also use multiple sequence alignments s7,97,98 $^{\text {. }}$

Estimates for rates of microdeletion events are, for all branches, approximately twofold higher than rates of microinsertion (Fig. 5b), suggesting a fundamental difference in the mechanisms that generate these mutations. Furthermore, there are substantial rate differences for each class of event between the various lineages. In particular, the rat lineage has accumulated microdeletions more rapidly than the mouse, while the opposite holds true for microinsertions. As with substitutions, both microinsertion and microdeletion rates are substantially slower in the human lineage. The size distribution of microindels (1-10 bp) on the rat branch was heavily weighted towards the smallest indels: $45 \%$ of indels are single bases, $18 \%$ are $2 \mathrm{bp}, 10 \%$ are $3 \mathrm{bp}, 8 \%$ are $4 \mathrm{bp}$, and so on, monotonically decreasing. Separate distributions for insertions and for deletions were similar, as were distributions of indel sizes on the mouse branch. 


\section{Male mutation bias}

As mouse and rat are similar in generation time and number of germline cell divisions ${ }^{99,100}$, we investigated a potential sex bias in different types of observed genome changes. We compared substitution and indel rates between the $\mathrm{X}$ chromosome and autosomes in ancestral repeat sites $(\sim 5 \mathrm{Mb}$ and $\sim 100 \mathrm{Mb}$ in total for $\mathrm{X}$ and autosomes, respectively $\left.{ }^{101}\right)$. We discovered that in rodents, small indels $(<50 \mathrm{bp}$ ) are male-biased, with a male-to-female rate ratio of $\sim 2.3$. This is in contrast to a recent study in primates, based on a substantially smaller data set, that indicates no sex bias in small indels ${ }^{102}$. Our male-to-female nucleotide substitution rate ratio in rodents is $\sim 1.9$, confirming earlier reports ${ }^{103,104}$. When substitution rates are compared for all sites aligned between mouse and rat ( $\sim 78 \mathrm{Mb}$ and $\sim 1,691 \mathrm{Mb}$, respectively), we again observe an approximately twofold excess of small indels and nucleotide substitutions originating in males compared with females ${ }^{101}$. Interestingly, the ratio in the number of cell divisions between the male and female germlines is also about two ${ }^{99,100}$, suggesting that these substitutions may arise from mutations that occur primarily during DNA replication.

\section{$\mathrm{G}+\mathrm{C}$ content and $\mathrm{CpG}$ islands}

The $\mathrm{G}+\mathrm{C}$ content of the rat varies significantly across the genome (Fig. 8a), and the distribution more closely resembles that of mouse than human. The variation in $\mathrm{G}+\mathrm{C}$ content is coupled with differences in the distribution of $\mathrm{CpG}$ islands-short regions that are associated with the $5^{\prime}$ ends of genes and gene regulation ${ }^{2,3,105}$, and that escape the depletion of CpG dinucleotides that occurs from
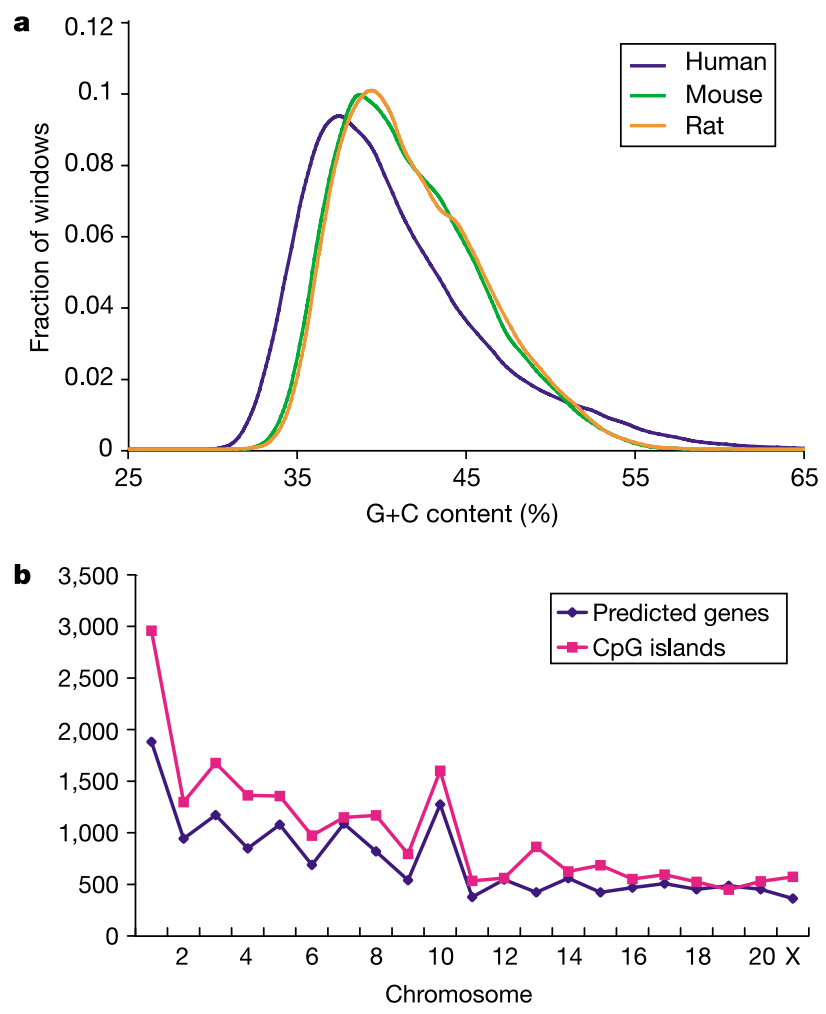

Figure 8 Base composition distribution analysis. a, The fraction of $20 \mathrm{~kb}$ non-overlapping windows ${ }^{3}$ with a given $\mathrm{G}+\mathrm{C}$ content is shown for human, mouse and rat. $\mathbf{b}$, The number of Ensembl-predicted genes per chromosome and the number of $\mathrm{CpG}$ islands per chromosome. The density of $\mathrm{CpG}$ islands averages 5.9 islands per $\mathrm{Mb}$ across chromosomes and 5.7 islands per $\mathrm{Mb}$ across the genome. Chromosome 1 has more $\mathrm{CpG}$ islands than other chromosomes, yet neither the island density nor ratio to predicted genes exceeds the normal distribution. The number of $\mathrm{CpG}$ islands per chromosome and the number of predicted genes are correlated $\left(R^{2}=0.96\right)$. deamination of methylated cytosine ${ }^{2,105}$. The $2.6 \mathrm{~Gb}$ rat genome assembly (including unmapped sequences) contains $15,975 \mathrm{CpG}$ islands in non-repetitive sequences of the genome. This is similar to the $15,500 \mathrm{CpG}$ islands reported in the $2.5 \mathrm{~Gb}$ mouse genome $\mathrm{e}^{3}$, but far fewer than the 27,000 reported in the human genome ${ }^{2,3,105}$.

A summary of the $\mathrm{CpG}$ island distributions by chromosome is given in Fig. 8b. Chromosome X, with a low $\mathrm{G}+\mathrm{C}$ content of $37.7 \%$, has the fewest islands (362) and the lowest density of islands (2.6 per $\mathrm{Mb})$. Chromosome 12 is at the other end of the range with a $\mathrm{G}+\mathrm{C}$ content of $43.5 \%$ and the highest density of $\mathrm{CpG}$ islands (11.5 islands per $\mathrm{Mb}$ ). This is similar to chromosome 10, with 11.3 islands per $\mathrm{Mb}$. The average density of $\mathrm{CpG}$ islands is 5.7 islands per $\mathrm{Mb}$ over the whole genome and 5.9 CpG islands per $\mathrm{Mb}$ averaged by chromosome, which is similar to the distribution in mouse ${ }^{3}$. Neither rodent genome shows the extreme outliers in CpG island density that are seen for human chromosome 19 (ref. 2). The density of $\mathrm{CpG}$ islands in the rat genome correlates positively with the density of predicted genes ( $R$ of 0.96 ) (Fig. 8b).

These data show that the overall changes in CpG island content predate the rat-mouse split and are consistent with the accelerated loss of CpG dinucleotides in rodents compared with humans ${ }^{105,106}$. It remains possible, however, that occurrences such as the greater number of human regions with extremely high $\mathrm{G}+\mathrm{C}$ content are due to distributional changes mostly in the primate, rather than in the rodent lineage.

\section{Shift in substitution spectra between mouse and rat}

The non-repetitive fraction of the rat genome is enriched for $\mathrm{G}+\mathrm{C}$ content relative to the mouse genome, by $\sim 0.35 \%$ over 1.3 billion nucleotides. This is a subtle but substantial difference that may be explained, at least in part, by differences in the spectra of mutation events that have accumulated in the mouse and rat lineages. We analysed all alignment columns in which substitution events can be assigned to either the mouse or the rat lineage, by virtue of a nucleotide match between human and only one rodent ${ }^{92}$; note that this is a small minority of substitutions. Of the $\sim 117$ million alignment columns meeting this criteria, $\sim 60$ million involve a change in the rat lineage versus $\sim 57$ million in the mouse, reflecting the increase in rates of point substitution in the rat lineage (Fig. 5b). While $50 \%$ of these changes in rat involve a substitution from an A/T to a G/C, these events constitute only $47 \%$ of all mouse changes. The complementary change, $\mathrm{G} / \mathrm{C}$ to $\mathrm{A} / \mathrm{T}$, exhibits relative excess in the mouse versus the rat lineage ( $38 \%$ versus $35 \%$, respectively). No substantial difference between changes that do not alter $\mathrm{G}+\mathrm{C}$ content is observed. In addition, this bias is not confined to particular transition or transversion events, nor can it be explained simply as a result of divergent substitution rates of CpG dinucleotides (data not shown). Thus, this shift appears to be a general change that results in an increase in $\mathrm{G}+\mathrm{C}$ content in the rat genome. Biochemical changes in repair or replication enzymes might be responsible, and the observation that recombination rates are slightly higher in rat than in mouse ${ }^{107}$ may suggest a role for G+C-biased mismatch repair ${ }^{108,109}$. However, population genetic factors, such as selection, cannot be ruled out.

\section{Evolutionary hotspots}

Comparison of the two rodent genomes, using human as outgroup, reveals regions that are conserved yet under different levels of constraint in mouse and rat. These regions may have distinct functional roles and contribute to species-specific differences. Analysis of the MAVID alignments ${ }^{110}$ revealed 5,055 regions $\geq 100 \mathrm{bp}$, in which there was at least a tenfold difference in the estimated number of substitutions per site on the mouse and rat branches. To avoid alignment problems and fast-evolving regions, the analysis was restricted to regions where the human branch had $<0.25$ substitutions per site ${ }^{111}$. These regions are enriched twofold in transcribed regions: $39 \%$ of mouse hotspots were found in the 
$18 \%$ of the mouse genome covered by RefSeq genes; and $17 \%$ of the rat hotspots were found in the $8 \%$ of the rat genome covered by RefSeq genes. Similar numbers are observed when examining coding exon and EST regions (not shown). Half of all hotspots in the mouse genome lie totally in non-coding regions. Many hotspots are several hundred bases long, with average length $190 \pm 86 \mathrm{bp}$. Future work aimed at identifying the genomic differences that contribute to phenotypic evolution may benefit from analyses such as these, which will become more powerful as the repertoire of mammalian genome sequences expands.

\section{Covariation of evolutionary and genomic features}

To illustrate the genomic and evolutionary landscape of a single rat chromosome in depth, we characterized features for rat chromosome 10 at $1 \mathrm{Mb}$ resolution (Fig. 9). This high-resolution analysis uncovered strong correlations between certain microevolutionary features ${ }^{89,92,98}$. Particularly strongly correlated are the local rates of microdeletion $\left(R^{2}=0.71\right.$; Fig. 9a), microinsertion $\left(R^{2}=0.56\right.$; Fig. $9 \mathrm{a})$, and point substitution $\left(R^{2}=0.86\right.$; Fig. $\left.9 \mathrm{~b}\right)$ between the two independent lineages of mouse and rat. In addition, microinsertion rates are correlated with microdeletion rates $\left(R^{2}=0.55\right.$; Fig. 9a). These strong correlations are also observed in an independent genome-wide analysis, both on the original data and after factoring out the effects of $\mathrm{G}+\mathrm{C}$ content (not shown, see Supplementary Information).

Perhaps surprisingly, substantially less correlation is seen between microindel and point substitution rates (compare Fig. 9a and b). The amount of correlation varies among chromosomes (not shown), but is generally weaker than the relationships mentioned above. Further studies will be required to determine whether local evolutionary pressures, which must have remained stable since the separation of the mouse and rat lineages, differentially drive microindel and point substitution rates.

We also find that the local point substitution rate in sites common to human, mouse and rat strongly correlates with that in rodentspecific sites $\left(R^{2}=0.57\right.$; Fig. 9b, blue line versus red/green). These two classes of sites, while interdigitated at the level of tens to thousands of bases, constitute sites that are otherwise evolutionarily independent. This result confirms that local rate variation is not solely determined by stochastic effects and extends, at high resolution, the previously documented regional correlation in rate between $4 \mathrm{D}$ sites and ancestral repeat sites ${ }^{3,96}$.

\section{Evolution of genes}

A substantial motivation for sequencing the rat genome was to study protein-coding genes. Besides being the first step in accurately defining the rat proteome, this fundamental data set yields insights into differences between the rat and other mammalian species with a complete genome sequence. Estimation of the rat gene content is possible because of relatively mature gene-prediction programs and rodent transcript data. Mouse and human genome sequences also allow characterization of mutational events in proteins such as amino acid repeats and codon insertions and deletions. The quality of the rat sequence also allows us to distinguish between functional genes and pseudogenes.

We estimate (on the basis of a subset) that $90 \%$ of rat genes
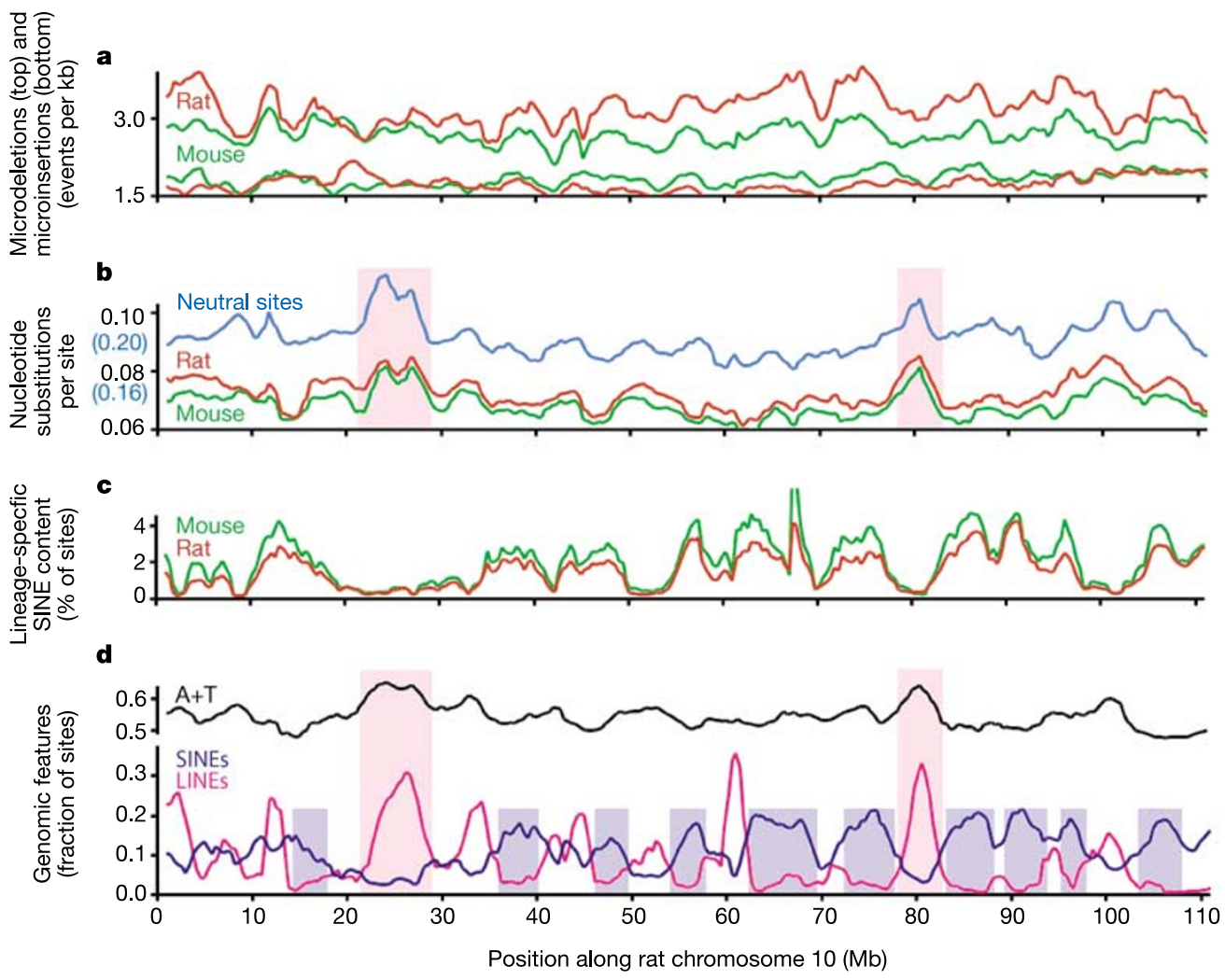

Figure 9 Variability of several evolutionary and genomic features along rat chromosome 10. a, Rates of microdeletion and microinsertion events (less than $11 \mathrm{bp}$ ) in the mouse and rat lineages since their last common ancestor, revealing regional correlations. b, Rates of point substitution in the mouse and rat lineages. Red and green lines represent rates of substitution within each lineage estimated from sites common to human, mouse and rat. Blue represents the neutral distance separating the rodents, as estimated from rodent-specific sites. Note the regional correlation among all three plots, despite being estimated in different lineages (mouse and rat) and from different sites (mammalian versus rodent-specific). c, Density of SINEs inserted independently into the rat or mouse genomes after their last common ancestor. $\mathbf{d}, \mathrm{A}+\mathrm{T}$ content of the rat, and density in the rat genome of LINEs and SINEs that originated since the last common ancestor of human, mouse and rat. Pink boxes highlight regions of the chromosome in which substitution rates, $A+T$ content and LINE density are correlated. Blue boxes highlight regions in which SINE density is high but LINE density is low. 
possess strict orthologues in both mouse and human genomes. Our studies also identified genes arising from recent duplication events occurring only in rat, and not in mouse or human. These genes contribute characteristic features of rat-specific biology, including aspects of reproduction, immunity and toxin metabolism. By contrast, almost all human 'disease genes' have rat orthologues. This emphasizes the importance of the rat as a model organism in experimental science.

\section{Construction of gene set and determination of orthology}

The Ensembl gene prediction pipeline $e^{112}$ predicted 20,973 genes with 28,516 transcripts and 205,623 exons (Methods). These genes contain an average of 9.7 exons, with a median exon number of 6.0. At least $20 \%$ of the genes are alternatively spliced, with an average of 1.3 transcripts predicted per gene. Of the $17 \%$ single exon transcripts, 1,355 contain frameshifts relative to the predicted protein and 1,176 are probably processed pseudogenes. Of the 28,516 transcripts, $48 \%$ have both $5^{\prime}$ and $3^{\prime}$ untranslated regions (UTRs) predicted and $60 \%$ have at least one UTR predicted.

These gene predictions considered homology to other sequences, including 26,949 rodent proteins, 4,861 non-rodent, vertebrate proteins, 7,121 rat complementary DNAs from RefSeq and EMBL, and 31,545 mouse cDNAs from Riken, RefSeq and EMBL. The majority $(61 \%)$ of transcripts are supported by rodent transcript evidence. When combined with additional private EST data, the fraction of genes supported by transcript evidence could be increased to $72 \%{ }^{113}$.

A number of other $a b$ initio (GENSCAN ${ }^{114}$, GENEID $^{115}$ ), similarity-based (FGENESH++; ref. 116) and comparative (SGP ${ }^{117}$, SLAM $^{118}$, TWINSCAN1 ${ }^{119-121}$ ) gene-prediction programs were used to analyse the rat genome. The number of genes predicted by these programs ranged from 24,500 to 47,000 , suggesting coding densities ranging from $1.2 \%$ to $2.2 \%$. The coding fraction of RefSeq genes covered by these predictions ranged from $82 \%$ to $98 \%$. Such comparative $a b$ initio programs using the rat genome were successfully used to identify and experimentally verify genes missed by other methods in rat $^{121}$ and human ${ }^{122}$. The predictions of these programs can be accessed through the UCSC genome browser and Ensembl websites.

RefSeq genes (20,091 human, 11,342 mouse and 4,488 rat) mapped onto genome assemblies with BLAT ${ }^{123}$ and the UCSC browser revealed that the number of coding exons per gene and average exon length were similar in the three species. Differences were observed in intron length, with an average of 5,338 bp in human, 4,212 bp in mouse and 5,002 bp in rat. These differences were also found in a smaller collection of 6,352 confidently mapped orthologous intron triads (see 'Conservation of intronic splice signals' section below): average intron lengths in this collection were $4,240 \mathrm{bp}$ in human, 3,565 bp in mouse and 3,638 bp in rat.

\section{Properties of orthologous genes}

Orthology relationships were predicted on the basis of BLASTp reciprocal best-hits between proteins of genome pairs (human-rat, rat-mouse and mouse-human) ${ }^{3}$ (Supplementary Information). Using these methods and the ENSEMBL prediction sets, 12,440 rat genes showed clear, unambiguous 1:1 correspondence with a gene in the mouse genome. This is an underestimate, because random sampling of different classes of rat genes with less stringent criteria for comparison to mouse always identified additional gene pairs. Errors arose from pseudogene misclassification, sequence loss, duplication or fragmentation in assemblies; and missing or inappropriate gene predictions, including coding-gene predictions from non-coding RNAs. Taking these errors into account, we estimate the true proportion of 1:1 orthologues in rat and mouse genomes to lie between 86 and 94\% (Methods). The remaining genes were associated with lineage-specific gene family expansions or contractions. These overall observations are consistent with a careful analysis of rat proteases showing that $93 \%$ of these genes have $1: 1$ orthologues in mouse ${ }^{124,125}$.

Surprisingly, a similar proportion (89 to 90\%) of rat genes possessed a single orthologue in the human genome. Because human represents an outgroup to the two rodents, it was expected that mouse and rat would share a higher fraction of orthologues. A close inspection of gene relationships indicates that these findings may suffer from incompleteness of rodent genome sequences, together with problems of misassembly and gene prediction within clusters of gene paralogues.

Further analysis of orthologous pairs considered the occurrence of nucleotide changes within protein-coding regions that reflected synonymous or non-synonymous substitutions. The majority of these studies measured evolutionary rates by determination of $K_{\mathrm{A}}$ (number of non-synonymous substitutions per non-synonymous site) and $K_{\mathrm{S}}$ (number of synonymous substitutions per synonymous site). $K_{\mathrm{A}} / K_{\mathrm{S}}$ ratios of less than 0.25 indicate purifying selection, values of 1 suggest neutral evolution, and values greater than 1 indicate positive selection ${ }^{126}$.

Evolutionary rates were first calculated from a reduced set of orthologue pairs that are embedded in orthologous genomic segments and are related by conservative values of $K_{\mathrm{S}}$ (Table 3 ) (Methods). A slight increase in median $K_{\mathrm{S}}$ values for rat-human as compared with mouse-human, was found, indicating that the rat lineage has more neutral substitutions in gene coding regions than the mouse lineage. Sequence conservation values were similar to those previously found using smaller data sets ${ }^{127,128}$, and the overall trend is consistent with results of other evolutionary rate analyses discussed above (Fig. 5).

Next, we investigated examples of rat genes shared with mouse, but with no counterparts in human. Such genes might be rapidly evolving so that homologues are not discernible in human, or they might have arisen from non-coding DNA, or their orthologues in the human lineage might have formed pseudogenes. Thirty-one Ensembl rat genes were collected that have no non-rodent homologues in current databases (Methods). These are twofold overrepresented among genes in paralogous gene clusters, and threefold over-represented among genes whose proteins are likely to be secreted. This is consistent with observations ${ }^{3}$ that clusters of paralogous genes, and secreted proteins, evolve relatively rapidly. Detailed examination of the 31 genes using PSI-BLAST determined that ten genes cannot be assigned homology relationships to experimentally described mammalian genes. These ten rodent-

\begin{tabular}{|c|c|c|c|}
\hline & Human-mouse & Human-rat & Mouse-rat \\
\hline $1: 1$ orthologue relationships & 11,084 & 10,066 & 11,503 \\
\hline Median $K_{\mathrm{S}}$ values* & $0.56(0.39-0.80)$ & $0.57(0.40-0.82)$ & $0.19(0.13-0.26)$ \\
\hline Median $K_{\mathrm{A}} / K_{\mathrm{S}}$ values* & $0.10(0.03-0.24)$ & $0.09(0.03-0.21)$ & $0.11(0.03-0.28)$ \\
\hline Median $\%$ amino acid identity ${ }^{*}$ & $88.0 \%(74.4-96.3 \%)$ & 88.3\% (75.9-96.4\%) & $95.0 \%+(88.0-98.7 \%)$ \\
\hline Median \% nucleotide identity* & $85.1 \%(77.4-90.0 \%)$ & $85.1 \%(77.8-89.9 \%)$ & $93.4 \%(89.2-95.7 \%)$ \\
\hline
\end{tabular}

†This value is consistent with previous findings (93.9\% in ref. 130). 
specific genes may have evolved particularly rapidly, or have noncoding DNA homologues, or be erroneous predictions.

The paucity of rodent-specific genes indicates that de novo invention of complete genes in rodents is rare. This is not unexpected, because the majority of eukaryotic protein-coding genes are modular structures containing coding and non-coding exons, splicing signals and regulatory sequences, and the chances of independent evolution and successful assembly of these elements into a functional gene are small, given the relatively short evolutionary time available since the mouse-rat split. However, individual rodent-specific exons may arise more frequently, particularly if the exon is alternatively spliced ${ }^{129}$. Applying a $K_{\mathrm{A}} / K_{\mathrm{S}}$ ratio test $^{130,131}$ to sequences that align only between rat and mouse, we identified 2,302 potential novel rodent-specific exons, with EST support, in BLASTZ alignments of rat and mouse sequences. None of these individual exons matched human transcripts, but approximately half $(1,116)$ appear to be present in alternative splice forms found in rodents. We speculate that these exons contain the few successful lineage-specific survivors of the constant process of gene evolution, by birth and death of individual exons.

\section{Indels and repeats in protein-coding sequences}

In contrast to small indels occurring in the bulk of the genome (above), indels within protein-coding regions are probably lethal, or deleterious and so are rapidly removed from the population by purifying selection. Indel rates within rat coding sequences were 50 fold lower than in bulk genomic DNA ${ }^{132}$. The whole genome excess of deletions compared with insertions (Fig. 5b) was also evident in coding sequences. The magnitude was less, with a genome-wide deletion-to-insertion ratio of 3.1:1 reducing to $1.7: 1$ in the rat. In mouse this value reduced from 2.5:1 to 1.1:1 (ref. 132). These data suggest that deletions are $\sim 16 \%$ more likely than insertions to be removed from coding sequences by selection.

Owing to the triplet nature of the genetic code, indels of multiples of three nucleotides in length $\left(3_{n}\right.$ indels $)$ are less likely to be deleterious. Direct comparison of $3_{n}$ indel rates between bulk DNA ( 0.77 indels per $\mathrm{kb}$ for mouse, 0.83 indels per $\mathrm{kb}$ for rat) and coding sequence $(0.087$ indels per $\mathrm{kb}$ for mouse and 0.084 indel per $\mathrm{kb}$ for rat) showed that $3_{n}$ indels were ninefold underrepresented in coding sequences. At least $44 \%$ of indels were duplicative insertion or deletion of a tandemly duplicated sequence, collectively termed sequence slippage ${ }^{132}$. Sequence slippage contributed approximately equally to observed insertions and deletions. The overall excess of deletions could be attributed specifically to an excess of non-slippage deletion over non-slippage insertion in both mouse and rat lineages ${ }^{132}$. Of the slippage indels, $13 \%$ were in the context of trinucleotide repeats $(n>2$, excluding the inserted or deleted sequence) which are known to be particularly prone to sequence slippage and encode homopolymeric amino acid $\operatorname{tracts}^{133,134}$.

To gain better understanding of dynamic changes in the length of homopolymeric amino acid tracts on gene evolution and disease susceptibility, we searched for other characteristics of amino acid repeat variation by analysing all size-five or longer amino acid repeats in a data set of 7,039 rat, mouse and human orthologous protein sequences ${ }^{135}$. Most species-specific amino acid repeats (80$90 \%$ ) were found in indel regions, and regions encoding speciesspecific repeats were more likely to contain tandem trinucleotide repeats than those encoding conserved repeats. This was consistent with the involvement of slippage in the generation of novel repeats in proteins and extended previous observations for glutamine repeats in a more limited human-mouse data set ${ }^{136}$.

The percentage of proteins containing amino acid repeats was $13.7 \%$ in rat, $14.9 \%$ in mouse and $17.6 \%$ in human ${ }^{135}$. The most frequently occurring tandem amino acid repeats were glutamic acid, proline, alanine, leucine, serine, glycine, glutamine and lysine. Using the same threshold size cut-off, tandem trinucleotide repeats were significantly more abundant in human than in rodent coding sequences, in striking contrast to the frequencies observed in bulk genomic sequences (29 trinucleotide repeats per $\mathrm{Mb}$ in rat, 32 repeats per $\mathrm{Mb}$ in mouse and 13 repeats per $\mathrm{Mb}$ in human, see discussion of the general simple repeat structure below). The conservation of human repeats was higher in mouse $(52 \%)$ than in rat $(46.5 \%)$, suggesting a higher rate of repeat loss in the rat lineage than the mouse lineage.

Functional consequences of these in-frame changes in rat, mouse and human were investigated ${ }^{132}$ through clustering of proteins based on annotation of function and cellular localization ${ }^{112}$, and mapping indels onto protein structural and sequence features. The rate that indels accumulated in secreted $\left(3.9 \times 10^{-4}\right.$ indels per amino acid) and nuclear $\left(4.0 \times 10^{-4}\right)$ proteins is approximately twice that of cytoplasmic $\left(2.4 \times 10^{-4}\right)$ and mitochondrial $\left(1.4 \times 10^{-4}\right)$ proteins. Likewise, ligand-binding proteins acquire indels $\left(3.1 \times 10^{-4}\right)$ at a higher rate than enzymes $\left(2.1 \times 10^{-4}\right)^{132}$. These trends exactly mirror those observed for amino acid substitution rates ${ }^{3}$, suggesting tight coupling of selective constraints between indels and substitutions. Transcription regulators showed the highest rate of indels $\left(4.3 \times 10^{-4}\right)$, a finding that may relate to the over-representation of homopolymorphic amino acid tracts in these proteins ${ }^{135}$.

Known protein domains exhibited 3.3-fold fewer indels than expected by chance, again paralleling nucleotide substitution rate differences between domains and non-domain sequences ${ }^{3}$. Of the protein-sequence and structural categories considered (transmembrane, protein domain, signal peptide, coiled coil and low complexity), the transmembrane regions were the most refractory to accumulating indels, exhibiting a sixfold reduction compared with that expected by chance. Low-complexity regions were 3.1-fold enriched, reflecting their relatively unstructured nature and enrichment in indel-prone trinucleotide repeats. Mapping of indels onto groups of known structures revealed that indels are $21 \%$ more likely to be tolerated in loop regions than the structural core of the $\operatorname{protein}^{132}$.

We observed that indel frequency and amino acid repeat occurrence both correlated positively with the $\mathrm{G}+\mathrm{C}$ coding sequence content of the local sequence environment ${ }^{132,135}$. This may be explained in part by the correlation of polymerase slippage-prone trinucleotide repeat sequences and $\mathrm{G}+\mathrm{C}$ content $^{135}$. There is also a positive correlation between $\mathrm{CpG}$ dinucleotide frequency and coding sequence insertions, but not deletions. This effect diminishes rapidly with increasing distance from the site of the insertion ${ }^{132}$.

\section{Transcription-associated substitution strand asymmetry}

A recent study reported a significant strand asymmetry for neutral substitutions in transcribed regions ${ }^{133}$. Within introns of nine genes, the higher rate of $A \rightarrow G$ substitutions over that of $T \rightarrow C$ substitutions, together with a smaller excess of $\mathrm{G} \rightarrow \mathrm{A}$ over $\mathrm{C} \rightarrow \mathrm{T}$ substitutions, leads to an excess of $\mathrm{G}+\mathrm{T}$ over $\mathrm{C}+\mathrm{A}$ on the coding strand (also verified on human chromosome 22). The authors ${ }^{133}$ hypothesized that the asymmetries are a byproduct of transcription-

\begin{tabular}{|c|c|c|}
\hline $\begin{array}{l}\text { Base frequencies on coding strand* } \\
(\mathrm{G}+\mathrm{T}) /(\mathrm{C}+\mathrm{A})\end{array}$ & $\begin{array}{c}\text { Rat genome } \\
1.060\end{array}$ & \\
\hline $\begin{array}{l}\text { Ratio of purine transitions to pyrimidine transitions } \dagger \\
\qquad \operatorname{Rate}(A \leftrightarrow G) / \text { Rate }(C \leftrightarrow T)\end{array}$ & $\begin{array}{c}\text { Rat-mouse } \\
1.036\end{array}$ & $\begin{array}{l}\text { Rat-human } \\
1.036\end{array}$ \\
\hline $\begin{array}{l}\text { Rate of transitions } \neq \\
\quad \text { Rate }(A \rightarrow G) / \operatorname{Rate}(T \rightarrow C) \\
\text { Rate }(G \rightarrow A) / \operatorname{Rate}(C \rightarrow T)\end{array}$ & $\begin{array}{l}\text { Rat } \\
1.058 \\
1.017\end{array}$ & $\begin{array}{c}\text { Mouse } \\
1.091 \\
1.00\end{array}$ \\
\hline
\end{tabular}


coupled repair in germline cells. Examining the three-way alignments of rat, mouse and human, we verified that the strand asymmetries for neutral substitutions exist in introns across the genome (Table 4).

Under the assumption of independence of sequence positions, large sample normal approximations to the binomial distribution allow us to test whether the fraction of $\mathrm{G}+\mathrm{T}$ exceeds 0.5 , and whether the rate at the numerator exceeds the rate at the denominator for each of the ratios in Table 4 . With the large amount of data provided by pooling introns genome-wide, the tests are all highly significant $\left(P\right.$ values $\left.<10^{-4}\right)$, except for the rate of $\mathrm{G} \rightarrow \mathrm{A}$ in mouse, which does not significantly exceed that of $\mathrm{C} \rightarrow \mathrm{T}$ $(P$ value $=0.6369)$. These asymmetries are also seen if the study is limited to ancestral repeat sites, excludes ancestral repeat sites, excludes CpG dinucleotides, is limited to positions flanked by sites that are identical in the aligned sequences (in the case of observations 2 and 3 in Table 4), or considers introns of RefSeq genes for human or mouse. Thus it appears that strand asymmetry of substitution events within transcribed regions of the genome is a robust genome-wide phenomenon.

\section{Conservation of intronic splice signals}

Using 6,352 human-mouse-rat orthologous introns from 976 genes (Methods), we examined the dynamics of evolution of consensus splice signals in mammalian genes. We found that intron class ${ }^{137}$ is extremely well conserved: we did not observe any U2 to U12 intron conversion, or vice versa, nor within U12 introns did we find any switching between the major AT-AC and GT-AG subtypes, although such events are documented at larger evolutionary distances $^{137}$. In contrast, conversions between canonical GT-AG and non-canonical GC-AG subtypes of $\mathrm{U} 2$ introns are not uncommon. Only $\sim 70 \%$ of GC-AG introns are conserved between human and mouse/rat, and only $90 \%$ are conserved between mouse and rat. Using human as the outgroup, we detected nine GT to GC conversions after divergence of mouse and rat (from 6,282 introns that were likely to have been GT-AG before human and rodents split), and two GC to GT conversions (from 34 GC-AG introns that probably predated the human and rodent split). These results give some indication of the degree to which mutation from $\mathrm{T}$ to $\mathrm{C}$ is tolerated in donor sites. The GC donor site appears to be better tolerated in introns with very strong donor sites, because in these introns the proportion of GC donor sites is $\sim 11 \%$, much higher than the $0.7 \%$ overall frequency of GC donor sites in U2 introns. Although we found a variety of other non-canonical configurations in U2 introns, very few are conserved, which suggests that most correspond to transient, evolutionarily unstable states, pseudogenes, or mis-annotations.

\section{Gene duplications}

Duplication of genomic segments represents a frequent and robust mechanism for generating new genes ${ }^{138}$. Because there were no compelling data showing rat-specific genes arising directly from non-coding sequences, we examined gene duplications to measure their potential contribution to rat-specific biology. A previous study showed that gene clusters in mouse without counterparts in human are subject to rapid, adaptive evolution ${ }^{3,139}$. We used two methods to identify recent gene duplications: methods that directly identified paralogous clusters, and methods that analysed genomic segmental duplications (see above).

Using the first approach, we found 784 rat paralogue clusters containing 3,089 genes (Methods). This was lower than in mouse (910 clusters/3,784 genes), but the difference probably reflects the larger number of gene predictions from the mouse assembly.

To investigate the timing of expansion of these individual families, we measured rates of local gene duplication and retention within clusters. BLAST is not suited to this ${ }^{140,141}$ and so we instead calculated the number of synonymous substitutions per synonymous site $\left(K_{\mathrm{S}}\right)$ between all pairs of homologous genes; constructed $K_{\mathrm{S}}$-derived phylogenetic trees; and predicted orthology or paralogy gene duplication events automatically from their topologies (Supplementary Information). The results showed that the neutral substitution rate varies among orthologues by approximately twofold (Fig. 10). This is similar to chromosomal variation shown previously by a study of mouse and human ancestral repeats ${ }^{3}$. Rates of change among ancestral gene duplications (those that predate the mouse-rat split) were relatively constant. Mouse-specific and rat-specific duplications occurred at similar rates, except for those with $K_{\mathrm{S}}<0.04$, which are reduced in mousespecific duplications (Fig. 10). More data are required to determine whether this reduction is a biological effect, as it might be accounted for by different protocols for assembling mouse and rat genomes, which differentially collapse areas of nearly identical sequence.

The rat paralogue pairs that probably arose after the rat-mouse split (12-24 Myr ago) have $K_{\mathrm{S}}$ values of $\leq 0.2$ (Table 3 ). We found $649 K_{\mathrm{S}}<0.2$ gene duplication events in rat, a lower number than is found in mouse (755). For both rodents, this represents a likelihood of a gene duplicating of between $1.3 \times 10^{-3}$ and $2.6 \times 10^{-3}$ every Myr. These are necessarily estimates, because gene deletions, conversions and pseudogene formation are not considered. Interestingly, the data are consistent with a previous estimate for Drosophila genes, but are an order of magnitude lower than an estimate for Caenorhabditis elegans genes ${ }^{140}$.

A subset of clusters have at least three gene duplications with $K_{\mathrm{S}}<0.2$ (Table 5). These are expected to be enriched in genes whose duplications persist as a consequence of positive selection. The group is dominated by genes involved in adaptive immune response and chemosensation ${ }^{87}$. Inspection of the $K_{\mathrm{S}}$-derived trees allowed us to infer the gene numbers in these clusters for the common ancestor of rat and mouse (that is, at $K_{\mathrm{S}}=0.2$ ), assuming no gene deletions or pseudogene generation (Table 5). Immunoglobulin, T-cell receptor $\alpha$-chain, and $\alpha_{2 \mathrm{u}}$-globulin genes appear to be duplicating at the fastest rates in the rat genome (Table 5). Since divergence with mouse, these rat clusters have increased gene content several-fold. This recapitulates previous observations that rapidly evolving and duplicating genes are over-represented in olfaction and odorant detection, antigen recognition and reproduction $^{142}$.

An examination of duplicated genomic segments showed this enrichment for most of the same genes and also elements involved in foreign compound detoxification (cytochrome P450 and carboxylesterase genes $)^{87}$. Together, these are exciting findings because each of these categories can easily be associated with a

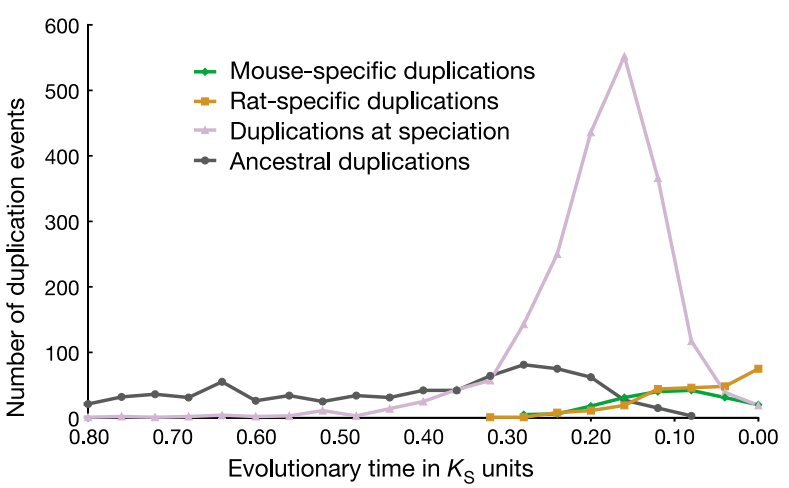

Figure 10 Variation in the frequency of gene duplications during the evolutionary histories of the rat and mouse. The sequence of gene duplication events was inferred from phylogenetic trees determined from pairwise estimates of genetic divergence under neutral selection $\left(K_{\mathrm{S}}\right.$, Methods). The median $K_{\mathrm{S}}$ value for mouse:rat 1:1 orthologues is 0.19 . This value corresponds to the divergence time of mouse and rat lineages. 
familiar feature of rat-specific biology, and further investigation could explain some differences between rats and their evolutionary neighbours.

\section{Conservation of gene regulatory regions}

As the third mammal to be fully sequenced, the rat can add significantly to the utility of nucleotide alignments for identifying conserved non-coding sequences ${ }^{143-147}$. This power increases roughly as a function of the total amount of neutral substitution represented in the alignment ${ }^{97,98}$, and rat adds about $15 \%$ to the human-mouse comparison (Fig. 5). Many conserved mammalian non-coding sequences are expected to have regulatory function, and can be predicted using further analyses based upon these alignments ${ }^{93,148-150}$

We applied such methods for detecting significantly conserved elements $^{97,151}$ and scoring regulatory potential ${ }^{148,152}$ to the genomewide human-mouse-rat alignments. Typical results show strong conservation for a coding exon, as well as for several non-coding regions (Fig. 11). For example, the intronic region in Fig. 11 contains $504 \mathrm{bp}$ that are highly conserved in human, mouse and rat. The last $100 \mathrm{bp}$ of this alignment block are identical in all three species. Peaks in regulatory potential score are correlated with conservation score, and in the highly conserved intronic segment, they are higher for the three-way regulatory potential score than for the two-way scores using human and just one rodent ${ }^{152}$. These data are illustrative, but form the foundation of ongoing efforts to identify genome sequences involved in gene regulation.

Requiring conservation among mammalian genomes greatly increases the specificity of predictions of transcription factor binding sites. Transcription factor databases such as TRANSFAC ${ }^{153}$ contain known transcription factor binding sites and some knowledge of their distribution, but simply searching a sequence with these motifs provides little discriminatory power. For example, all of the 85 known regulatory elements ${ }^{148}$ and 151 functional promoters ${ }^{154}$ have TRANSFAC matches, but so do $99 \%$ of the 2,049,195 mammalian ancestral repeats, most representing false-positive predictions. The introduction of conservation as a criterion for regulatory element identification greatly increases specificity, with only a modest cost in sensitivity. If we insist that the TRANSFAC matches be present and orthologously aligned in all three specieshuman, mouse and rat-then only 268 matches are recorded in ancestral repeats $(0.01 \%)$, while $63(74 \%)$ of the above matches in known regulatory elements and $121(80 \%)$ in functional promoters are retained. Overall, using a set of 164 weight matrices for 109 transcription factors extracted from TRANSFAC ${ }^{153}$, we find 186,792,933 matches in the April 2003 reference human genome sequence, but this was reduced to only $4,188,229$ by demanding conservation in the human-mouse-rat three-way alignments. This is a 44 -fold increase in specificity.

We examined one region in more detail: a complex cis-regulatory region consisting of a $4,000 \mathrm{bp}$ segment containing two regulatory modules, hypersensitive sites 2 and 3 from the locus control region of the HBB complex ${ }^{155-157}$. Considerable experimental work has identified six functional binding sites for the transcription factor GATA-1 in this segment. Requiring that matches to GATA-1 binding sites be conserved in all three species and occur within regions of strong regulatory potential is sufficient to find these six functional binding sites, and only these six, in the $4,000 \mathrm{bp}$ segment. Thus, in this example we observed complete sensitivity and specificity by requiring this level of conservation.

\section{Pseudogenes and gene loss}

To complement the identification and analysis of protein-coding regions, we sought to examine rat pseudogenes. Using a previously described method ${ }^{158,159}$, we found 18,755 pseudogenes in intergenic regions. Pseudogenes are normally not subjected to selective con-

\begin{tabular}{|c|c|c|c|c|c|c|c|}
\hline Table 5 & cent gene duplicat & is $\left(K_{\mathrm{S}}<0.2\right)$ in the $r$ & lineage & & & & \\
\hline $\begin{array}{l}\text { Cluster } \\
\text { ID }\end{array}$ & $\begin{array}{c}\text { Recent duplication } \\
\text { events }\end{array}$ & $\begin{array}{c}\text { Numbers of genes } \\
\text { involved }\end{array}$ & $\begin{array}{l}\text { Extant cluster } \\
\text { size }\end{array}$ & $\begin{array}{c}\text { Ancestral cluster } \\
\text { size }\end{array}$ & Chromosome & Annotation & Process \\
\hline 249 & 38 & 53 & 60 & 22 & 4 & Immunoglobulin $к$-chain $\mathrm{V}$ & Immunity \\
\hline 640 & 38 & 47 & 53 & 15 & 15 & TCR $\alpha$-chain V & Immunity \\
\hline 346 & 25 & 35 & 44 & 15 & 6 & Immunoglobulin heavy chain $\mathrm{V}$ & Immunity \\
\hline 190 & 22 & 42 & 168 & 146 & 3 & Olfactory receptor & Chemosensation \\
\hline 578 & 16 & 28 & 59 & 43 & 13 & Olfactory receptor & Chemosensation \\
\hline 400 & 15 & 26 & 82 & 67 & 8 & Olfactory receptor & Chemosensation \\
\hline 743 & 15 & 21 & 37 & 22 & 20 & Olfactory receptor & Chemosensation \\
\hline 72 & 12 & 22 & 102 & 90 & 1 & Olfactory receptor & Chemosensation \\
\hline 500 & 12 & 18 & 32 & 20 & 10 & Olfactory receptor & Chemosensation \\
\hline 51 & 6 & 7 & 16 & 10 & 1 & Glandular kallikrein & Reproduction? \\
\hline 256 & 6 & 8 & 10 & 4 & 4 & Vomeronasal receptor V1R & Chemosensation \\
\hline 488 & 6 & 10 & 11 & 5 & 10 & Olfactory receptor & Chemosensation \\
\hline 644 & 6 & 10 & 14 & 8 & 15 & Granzyme serine protease & Immunity \\
\hline 4 & 5 & 6 & 9 & 4 & 1 & Trace amine receptor, GPCR & Neuropeptide receptors? \\
\hline 248 & 5 & 9 & 15 & 10 & 4 & Vomeronasal receptor V1R & Chemosensation \\
\hline 393 & 5 & 10 & 31 & 26 & 8 & Olfactory receptor & Chemosensation \\
\hline 522 & 5 & 8 & 19 & 14 & 10 & Keratin-associated protein & Epithelial cell function \\
\hline 550 & 5 & 8 & 17 & 12 & 11 & Olfactory receptor & Chemosensation \\
\hline 635 & 5 & 9 & 20 & 15 & 15 & Olfactory receptor & Chemosensation \\
\hline 79 & 4 & 8 & 38 & 34 & 1 & Olfactory receptor & Chemosensation \\
\hline 88 & 4 & 6 & 11 & 7 & 1 & Olfactory receptor & Chemosensation \\
\hline 109 & 4 & 7 & 43 & 39 & 1 & Olfactory receptor & Chemosensation \\
\hline 294 & 4 & 5 & 5 & 1 & 5 & $\alpha_{2 u}$-globulin & Chemosensation \\
\hline 310 & 4 & 5 & 11 & 7 & 5 & Olfactory receptor & Chemosensation \\
\hline 353 & 4 & 7 & 13 & 9 & 7 & Olfactory receptor & Chemosensation \\
\hline 399 & 4 & 5 & 6 & 2 & 8 & Ly6-like urinary protein & Chemosensation? \\
\hline 638 & 4 & 6 & 6 & 2 & 15 & RNase A & Immunity \\
\hline 690 & 4 & 6 & 21 & 17 & 17 & Prolactin paralogue & Reproduction \\
\hline 239 & 3 & 6 & 6 & 3 & 4 & Prolactin-induced protein & Reproduction \\
\hline 253 & 3 & 4 & 5 & 2 & 4 & Camello-like $N$-acetyltransferase & Developmental regulator \\
\hline 274 & 3 & 6 & 20 & 17 & 4 & Ly-49 lectin natural killer cell protein & Immunity \\
\hline 297 & 3 & 4 & 5 & 2 & 5 & Interferon- $\alpha$ & Immunity \\
\hline 523 & 3 & 4 & 6 & 3 & 10 & Keratin-associated protein & Epithelial cell function \\
\hline 746 & 3 & 5 & 6 & 3 & 20 & MHC class $1 \mathrm{~b}(\mathrm{M} 10)$ & Chemosensation \\
\hline
\end{tabular}


straint and therefore accumulate sequence modifications neutrally. Indeed, nearly all of our identified pseudogenes ( $97 \pm 3 \%)$ evolved under neutrality according to a $K_{\mathrm{A}} / K_{\mathrm{S}}$ test, and therefore are consistent with being pseudogenic.

We classified these pseudogenes according to whether they arose from retrotransposition, in which case they integrated into the genome randomly, or whether they arose from tandem duplication and neutral sequence substitution. Using human-rat synteny, we found that $80 \%$ of pseudogenes exhibited no significant similarity to the corresponding human orthologous region, and therefore were considered retrotransposed, processed pseudogenes. The total pseudogene count, and processed pseudogene proportion, are consistent with those found for human ${ }^{158,159}$. These numbers are greater than those previously reported for mouse ${ }^{3,4}$. However, reanalysis using the method employed here detects a similar pseudogene number $(20,000)$ to that found for human and rat. This suggests that the rate of pseudogene creation is similar among these mammals.

As with the human genome $e^{159,160}$, the largest group of rat pseudogenes (totalling 2,188), according to InterPro ${ }^{161}$, consists of ribosomal protein genes. Other large rat pseudogene families arose from olfactory receptors (552, see below), glyceraldehyde-3-phosphate dehydrogenase (GAPDH) (251), protein kinases (177), and RNA binding RNP-1 proteins (174). Pseudogenes homologous to a meiotic spindle-associated protein-spindlin ${ }^{162}$-are particularly numerous in rat (at least 53 copies) compared with mouse (approximately three copies). This suggests that spindlin pseudogenes may have distributed rapidly by a recently active transposable element.

We investigated the much-studied metabolic enzyme GAPDH $^{3,163}$, and observed that: (1) the GAPDS gene arose from a duplication of the GAPDH gene; (2) biogenesis of the GAPDH pseudogenes has been occurring steadily over time both before and

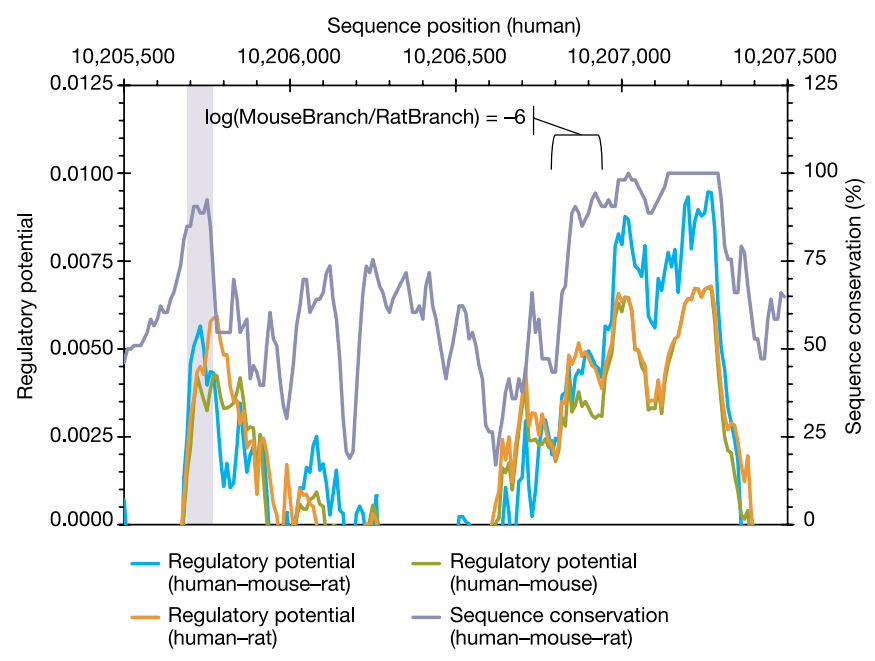

Figure 11 Close-up of PEX14 (peroxisomal membrane protein) locus on human chromosome 1 (with homologous mouse chromosome 4 and rat chromosome 5). Conservation score computed on three-way human-mouse-rat alignments (parsimony $P$ values ${ }^{151}$ ) presents a clear coding exon peak (grey bar) and very high values in a $504 \mathrm{bp}$ non-coding, intronic segment (right; last $100 \mathrm{bp}$ of alignment are identical in all three organisms). The latter segment showed a striking difference between the inferred mouse and rat branch lengths ${ }^{110,111,222:}$ : the grey bracket corresponds to a phylogenetic tree where the logarithm of mouse to rat branch-length ratio is -6 . Regulatory potential scores $^{148,152}$ that discriminate between conserved regulatory elements and neutrally evolving DNA are calculated from three-way (human-mouse-rat) and two-way (humanrodent) alignments. Here the three-way regulatory potential scores are enhanced over the two-way scores. after rodent-human and mouse-rat divergence; and (3) the GAPDS gene has undergone little retrotransposition in all three genomes compared with its relative, the GAPDH gene (consistent with respective gene-expression levels in the germ line).

\section{In situ loss of rat genes}

As an organism evolves, its need for certain genes may be reduced, or lost, owing to changes in its ecological niche. Loss of selective constraints leads to accumulation of nonsense and/or frameshift mutations without retrotransposition or duplication. These nonprocessed pseudogenes are interesting because they link environmental changes to genomic mutation events. However, predicted pseudogenes with disrupted reading frames might also be indicative of errors in genome sequence or assembly. By constraining the search to orthologous genomic regions, we identified 14 rat putative non-processed pseudogenes (Table 6) with apparently functional, single human and mouse orthologues. Half of these contain one inframe stop or frameshift, whereas the remainder contain more. We expect this number of identified pseudogenic orthologues to be conservative because the methods employed required high fidelity of both gene prediction and orthologue identification in all three species (Methods).

Nevertheless, as only 14 recently evolved pseudogene candidates were identified, this indicates that the genome sequence and assembly (Rnor3.1) is of high quality. The improved quality of the most recent assembly is underscored by 11 additional candidate pseudogenes, predicted from rat assembly Rnor2.1, that are apparently functional, full-length genes in Rnor3.1. Consequently, some of the current 14 candidates, in particular those that are involved in fundamental processes of eukaryotic biology, may yet be 'repaired' by sequence changes in future assemblies, and thus be recognized as genic. However, genes associated with innate immunity (which is particularly susceptible to change via adaptive evolution), such as Forssman glycolipid synthetase and complement factor I, may yet be found to survive as true pseudogenes in the rat.

\section{Non-coding RNA genes}

We investigated the abundance and distribution of non-coding (nc)RNAs in rat. Cytoplasmic transfer ( $\mathrm{t}$ )RNA gene identification in rodents is complicated by tRNA-derived identifier (ID) short interspersed nucleotide (SINEs) (B2 and ID). tRNAscan-SE predicted 175,943 tRNAs (genes and pseudogenes); however, the majority $(175,285)$ were SINEs identified by RepeatMasker. This is far greater than the number found in mouse $(24,402 / 25,078)$ or human (25/636). Of the remaining 666 predictions, 163 were annotated as tRNA pseudogenes and four were annotated as undetermined by tRNAscan-SE. An additional 68 predictions were removed because their best database match in either human, mouse or rat tRNA databases matched tRNAs with either a different amino acid or anticodon (violating the wobble rules that specify the distinct anticodons expected). The total of 431 tRNAs (including a single selenocysteine tRNA) identified in the rat genome is comparable to that for mouse-435 tRNAs (version $\mathrm{mm} 2$ from the UCSC genome browser) - and human-492 tRNAs (from the genomic tRNA database, http://rna.wustl.edu/GtRDB/Hs/). These three species share a core set of approximately 300 tRNAs, using a cutoff of $\geq 95 \%$ sequence identity and $\geq 95 \%$ sequence length.

A total of 454 ncRNAs (other than tRNAs) were identified by sequence comparison to known ncRNAs (Supplementary Information). These include 113 micro- (mi)RNAs, five ribosomal RNAs, 287 small nucleolar (sno)RNAs and small nuclear (sn)RNAs, 49 various other ncRNAs such as signal recognition particle (SRP) RNA, 7SK RNA, telomerase RNA, RNase P RNA, brain-specific repetitive (bsr)RNA, non-coding transcript abundantly expressed in brain (ntab)RNA, small cytoplasmic (sc)RNA and 626 pseudogenes. Complete $18 \mathrm{~S}$ and $28 \mathrm{~S}$ rRNA genes and more rRNAs were not identified, presumably owing to assembly issues. 
Table 6 Candidate rat pseudogenes, orthologous to mouse and human functional genes

\begin{tabular}{|c|c|c|c|c|c|}
\hline Mouse gene & Human gene & Strand & Rat genome coordinates* & Frameshifts/stops $†$ & Annotation \\
\hline ENSMUSG00000013611 & ENSG00000174226 & + & 7:92752590-92807556 & $1 / 0$ & Sorting nexin \\
\hline ENSMUSG00000024364 & ENSG00000158402 & + & 18:62742414-62770427 & $2 / 0$ & Dual-specificity phosphatase CDC25c \\
\hline ENSMUSG00000026293 & ENSG00000077044 & + & 9:95634847-95692601 & $1 / 0$ & Diacylglycerol kinase $\delta$ \\
\hline ENSMUSG00000026785 & ENSG00000160447 & + & 3:9210762-9229984 & $5 / 0$ & Protein kinase PKN $\beta$ \\
\hline ENSMUSG00000026829 & ENSG00000148288 & + & 3:7662414-7664521 & $2 / 2$ & Forssman glycolipid synthetase \\
\hline ENSMUSG00000027426 & ENSG00000125846 & + & $3: 125918806-125924149$ & $1 / 1$ & Zinc finger protein 133 \\
\hline ENSMUSG00000028000 & ENSG00000138799 & - & $2: 221272797-221304350$ & $1 / 0$ & Complement factor I \\
\hline ENSMUSG00000029203 & ENSG00000078140 & - & 14:44385206-44441888 & $1 / 0$ & Ubiquitin-protein ligase E2 (HIP2) \\
\hline ENSMUSG00000030270 & ENSG00000144550 & - & 20:8332585-8362331 & $3 / 0$ & Copine (membrane trafficking) \\
\hline ENSMUSG00000035449 & ENSG00000167646 & + & $1: 67374986-67381472$ & $1 / 0$ & Cardiac troponin I \\
\hline ENSMUSG00000037029 & ENSG00000105261 & - & 1:82728049-82730272 & $1 / 0$ & Zinc finger protein 146 \\
\hline ENSMUSG00000037432 & ENSG00000158142 & + & $9: 42465695-42498651$ & $1 / 1$ & Dysferlin-like protein \\
\hline ENSMUSG00000039660 & ENSG00000167137 & - & 3:9320401-9326997 & $4 / 0$ & Similar to yeast YMR310c RNA-binding protein \\
\hline ENSMUSG00000042653 & ENSG00000137634 & + & 8:49938446-49939091 & $1 / 0$ & Brush border $61.9 \mathrm{kDa}$-like protein \\
\hline
\end{tabular}

†Mouse genes were used as templates for predicting rat pseudogenes.

\section{Evolution of transposable elements}

Most interspersed repeats are immobilized copies of transposable elements that have accrued substitutions in proportion to their time spent fixed in the genome (for introduction ${ }^{2,3,164-167}$ ). About $40 \%$ of the rat genome draft is identified as interspersed repetitive DNA derived from transposable elements, similar to that for the mouse ${ }^{3}$ (Table 7) and lower than for the human (almost $50 \%{ }^{2}$ ). The latter difference is mainly due to the lower substitution rate in the human lineage, which allows us to recognize much older (Mesozoic) sequences as interspersed repeats. Almost all repeats are derived from retroposons, elements that procreate via reverse transcription of their transcripts. As in mouse, there is no evidence for activity of DNA transposons since the rat-mouse split. Many aspects of the rat and the mouse genomes' repeat structure are shared; here we focus on the differences.

\section{LINE-1 activity in the rat lineage}

The long interspersed nucleotide element (LINE)-1 (L1) is an autonomous retroelement, containing an internal RNA polymerase II promoter and two open reading frames (ORFs). The ORF1 product is an RNA binding protein with chaperone-like activity, suggesting a role in mediating nucleic acid strand transfer steps during L1 reverse transcription ${ }^{168}$, whereas ORF2 encodes a protein with both reverse transcriptase and DNA endonuclease activity. LINEs are characteristically $5^{\prime}$ truncated so that only a small subset extends to include the promoter region and can function as a source for more copies.

Many classes of LINE-like elements exist, but only L1 has been active in rodents. Over half a million copies, in variable stages of decay, comprise $22 \%$ of the rat genome. Although $10 \%$ of the human genome is comprised of L1 copies introduced before the rodent-primate split, owing to the fast substitution rate in the rodent lineage only $2 \%$ of the rat genome could be recognized as such. Thus, probably well over one-quarter of all rat DNA is derived directly from the L1 gene.

Following the mouse-rat split, L1 activity appears to have increased in rat. The $3^{\prime}$ UTR sequences defined six rat-specific L1 subfamilies, represented by 150,000 copies that cover $12 \%$ of the rat genome. L1 copies accumulated over the same period in mouse cover only $10 \%$ of the genome (Table 7 ). This higher accumulation of L1 copies could explain some of the size difference of the rat and mouse genome.

In addition to the traditional $\mathrm{L} 1$ elements, there are 7,500 copies

\begin{tabular}{|c|c|c|c|c|c|c|}
\hline & & & Rat & & & \\
\hline & Copies $\left(\times 10^{3}\right)$ & Total length (Mb) & Fraction of genome (\%) & Lineage-specific (\%) & Fraction of genome (\%) & Lineage-specific (\%) \\
\hline LINEs & 657 & 594.0 & 23.11 & 11.70 & 20.10 & 9.74 \\
\hline LINE-1 & 597 & 584.2 & 22.73 & 11.70 & 19.65 & 9.74 \\
\hline LINE-2 & 48 & 8.4 & 0.33 & - & 0.38 & - \\
\hline L3/CR1 & 11 & 1.4 & 0.06 & - & 0.06 & - \\
\hline SINES & 1,360 & 181.3 & 7.05 & 1.52 & 7.78 & 1.80 \\
\hline B1(Alu) & 384 & 42.3 & 1.65 & 0.16 & 2.53 & 0.92 \\
\hline B4(ID_B1) & 359 & 55.4 & 2.15 & 0.00 & 2.25 & 0.00 \\
\hline $\mathrm{ID}$ & 225 & 19.6 & 0.76 & 0.54 & 0.20 & 0.00 \\
\hline B2 & 328 & 55.2 & 2.15 & 0.68 & 2.29 & 0.74 \\
\hline MIR & 109 & 13.0 & 0.51 & - & 0.56 & - \\
\hline LTR elements & 556 & 232.4 & 9.04 & 1.84 & 10.28 & 2.85 \\
\hline ERV_class I & 40 & 24.9 & 0.97 & 0.56 & 0.79 & 0.36 \\
\hline ERV_class II & 141 & 83.4 & 3.24 & 1.02 & 4.13 & 1.73 \\
\hline ERVL (III) & 74 & 21.6 & 0.84 & 0.04 & 1.08 & 0.23 \\
\hline MaLRs & 302 & 102.5 & 3.99 & 0.22 & 4.27 & 0.53 \\
\hline DNA elements & 108 & 20.9 & 0.81 & - & 0.86 & - \\
\hline Charlie(hAT) & 80 & 14.8 & 0.58 & - & 0.60 & - \\
\hline Tigger(Tc1) & 18 & 4.0 & 0.16 & - & 0.17 & - \\
\hline Unclassified & 14 & 7.3 & 0.28 & - & 0.37 & - \\
\hline Total & 2,690 & 1,036 & 40.31 & 14.90 & 39.45 & 14.26 \\
\hline Small RNAs & 8 & 0.6 & 0.03 & 0.01 & 0.03 & 0.01 \\
\hline Satellites & 14 & 6.4 & 0.25 & $?$ & 0.31 & $?$ \\
\hline Simple repeats & 897 & 61.1 & 2.38 & ? & 2.41 & $?$ \\
\hline
\end{tabular}

Data for Rnor3.1 and October 2003 mouse (MM4), excluding Y chromosome, using the 17 December 2003 version of RepeatMasker. To highlight the differences between rat and mouse repeat content, columns 5 and 7 show the fractions of the genomes comprising lineage-specific repeats. The LINE-1 numbers include all HAL1 copies, whereas all BC1 scRNA and >10\% diverged tRNA-Ala matches, far more common than other small RNA pseudogenes and closely related to ID, have been counted as ID matches. 
$(10 \mathrm{Mb})$ of a non-autonomous element that is derived from L1 by deletion of most of its ORF2. A similar element, active in Mesozoic times, has been called HAL1 (for Half-a-LINE) ${ }^{164}$. Given their low divergence, we conclude that the currently identified HAL1-like elements operated only a few million years ago in the mouse lineage (MusHAL1) and still propagate in the rat genome (RNHAL1). RNHAL1 contains only an ORF1, whereas MusHAL1 encoded an endonuclease as well, although no reverse transcriptase. The $5^{\prime}$ 2,600 bases of RNHAL1 are $98 \%$ identical to the currently active L1 in rat (L1_Rn or L1mlvi2 $\left.{ }^{169}\right)$. Unlike ancient HAL1 elements, which shared the $3^{\prime}$ UTR with a contemporary L1, the $3^{\prime}$ end of RNHAL1 is unrelated to other repeats. The repeated origin and high copy number of HAL1s suggest that the ORF1 product, which binds strongly to its messenger $\mathrm{RNA}^{168}$, may render this transcript a superior target for L1-mediated reverse transcription. In this way HAL1 resembles the non-autonomous, endogenous retrovirusderived MaLR elements (below), which, for over 100 million years, retained only the retroviral gag ORF that encodes an RNA binding protein. A potential advantage of HAL1 over L1 is its shorter length, which, considering the usual $5^{\prime}$ truncation of copies, increases the chance that a copy may include the internal promoter elements and become a source gene.

\section{Different activity of SINEs in the rat and mouse lineage}

The most successful usurpers of the L1 retrotransposition machinery, however, are SINEs. These are small RNA-derived sequences with an internal RNA polymerase III promoter. Recently, the human Alu SINE has been experimentally proven to be transposed by $\mathrm{L}^{170}$. Most SINEs share the $3^{\prime}$ end with their associated LINE elements, like the Mesozoic mammalian LINE-2 (L2) and MIR pair, increasing the efficiency with which a LINE reverse transcriptase recognizes the $3^{\prime}$ end of a dependent SINE. However, L1 does not show sequence specificity and rodent and primate SINE sequences are unrelated to L1. Although any transcript can be retroposed, as can be seen from the numerous processed pseudogenes in mammalian genomes, L1-dependent SINEs probably have features that make them especially efficient targets of the L1 reverse transcriptase.

Although before the radiation of most mammalian orders L1 was at least as active as L2, the L2-dependent MIR was the only known (and very abundant) SINE of that time. All of the currently active SINEs in different mammalian orders appear to have arisen after the demise of L2 (and consequently MIR), as though an opportunity (or necessity) arose for the creation and expansion of other SINEs.

Four different SINEs are distinguished in rat and mouse. The B1 element seems to share its origin from a 7SL RNA gene with the primate $\mathrm{Alu}^{171}$. This probably happened just before the rodentprimate split and after the speciation from most other eutherians, where Alu/B1 elements are not known. The other SINEs are rodentspecific and have tRNA-like internal promoter regions. ID elements consist only of this tRNA-like region, which in older ID copies closely match an Ala-tRNA from which it may have been derived. B4 resembles a fusion of an ID and B1 SINE. Finally, B2 has a tRNA-like region of unknown affiliation followed by a unique $120 \mathrm{bp}$ region.

The fortunes of these SINEs during mouse and rat evolution have been different (Fig. 12). B4 probably became extinct before the mouse-rat speciation, while $\mathrm{B} 2$ has remained productive in both lineages, scattering $>100,000$ copies in each genome after this time. Interestingly, the fate of the B1 and ID SINEs has been opposite in rat and mouse. While B1 is still active in mouse, having left over 200,000 mouse-specific copies in its trail, the youngest of the 40,000 rat-specific B1 copies are $6-7 \%$ diverged from their source, indicating a relatively early extinction in the rat lineage. On the other hand, after the mouse-rat split only a few hundred ID copies may have inserted in mouse, whereas this previously minor SINE ( $\sim 60,000$ copies predate the speciation) increased its activity in rat to produce 160,000 ID copies.

\section{Co-localization of SINEs in rat and mouse}

Despite the different fates of SINE families, the number of SINEs inserted after speciation in each lineage is remarkably similar: $\sim 300,000$ copies. Reminiscent of the replacement of MIR by L1 driven SINEs, it seems that the demise of B1 in rat allowed the expansion of IDs. Moreover, these independently inserted and unrelated SINEs (ID and B1 share only a mechanism of retroposition) accumulated at orthologous sites: the density of ratspecific SINEs in $14,243 \sim 100 \mathrm{~kb}$ windows in the rat genome is highly correlated $\left(R^{2}=0.83\right)$ with the density of mouse-specific SINEs in orthologous regions in mouse. To avoid including elements fixed before the speciation, only SINEs labelled lineagespecific on the basis of subfamily assignment (Methods ${ }^{89}$ ) were tallied with a divergence from the consensus that was well below the 9\% average for neutral sites (Fig. 5). These data corroborate and refine the observation of a strong correlation between the location of primate- and rodent-specific SINEs in $1 \mathrm{Mb}$ windows ${ }^{3}$. At $100 \mathrm{~kb}$, no correlation is seen for interspersed repeats other than SINEs.

Insertions of SINEs at the same location in different species have been reported ${ }^{172-174}$, and the correlation could reflect the existence of conserved hotspots for SINE insertions. However, only five of $\sim 800$ human specific Alu elements have an Alu inserted within $100-200$ bp in any of six other primate lineages ${ }^{174-176}$. Likewise, gene conversions of shared Alus into lineage-specific copies were observed five times in the same set, too low a level to contribute significantly to the observed correlation ${ }^{174-176}$.

Figure 9c displays the lineage-specific SINE densities on rat chromosome 10 and in the mouse orthologous blocks, showing a stronger correlation than any other feature. The cause of the unusual distribution patterns of SINEs, accumulating in gene-rich regions where other interspersed repeats are scarce, is apparently a conserved feature, independent of the primary sequence of the SINE and effective over regions smaller than isochores.

In the human genome, the most recent (unfixed) Alus are distributed similarly to L1, whereas older copies gradually take on the opposite distribution of SINEs ${ }^{2,164}$. This suggested that SINEs insert in the same places as LINEs, and that the typical SINE pattern is due to selection (or deletion bias) rather than a mechanistic insertion bias shared by all (unrelated) SINEs, but not by LINEs that use the same insertion process. This led to a proposal that SINEs are preferentially maintained in regions where they can easily be expressed $^{2,164}$ : if so, this could be the local feature conserved between mammalian genomes that leads to the strong correlation of local SINE densities in different mammals. However, we did not observe this temporal shift in SINE distribution pattern in mouse, nor currently in the rat genome, despite a considerable effort to define the potentially unfixed SINEs in both species (see ref. 89 for details). The observations in human could reflect a recent change in Alu behaviour, which would necessitate another explanation for the contrary insertion-preference of older Alus and all other SINEs.

Some regions of high LINE content coincide with regions that exhibit both higher AT content and an increased rate of point substitution (Fig. 9, pink rectangles). In a genome-wide analysis, LINE content correlates strongly with substitution rates, and about $80 \%$ of this correlation is explained by higher rates in AT-rich regions ${ }^{89}$. SINE density shows the opposite correlation both on chromosome 10 (Fig. 9) and genome-wide ${ }^{89}$.

These phenomena, in conjunction with an overall trend in substitution rates towards AT-richness, suggest a model in which quickly evolving regions accumulate a higher-than-average AT content, which attracts LINE elements. Although distinct causeeffect relationships such as this remain largely speculative, these results reinforce the idea that local genomic context strongly shapes local genomic features and rates of evolution.

\section{Endogenous retroviruses and derivatives}

The other major contributors to interspersed repeats in the rodent 
genome are retrovirus-like elements. These have several $100 \mathrm{bp}$ long terminal repeats (LTRs) with transcriptional regulatory sequences that flank an internal sequence that, in autonomous elements, encodes all proteins necessary for retrotransposition. All mammalian LTR elements are endogenous retroviruses (ERVs) or their nonautonomous derivatives. They fall into three groups, of which representatives in mouse are: murine leukaemia virus (MuLV) (class I), intracisternal A-particle (IAP) and MMTV (class II), and MERVL (class III).

The most productive retrovirus in mammals has been the class III element ERV-L, primarily through its ancient non-autonomous derivatives, called MaLRs, with 350,000 copies occupying $~ 5 \%$ of the rat genome (Table 7). Human ERV-L and MaLR copies are $>6 \%$ diverged from their reconstructed source genes and must have died out around the time of human speciation from New World monkeys. In mouse, several thousand almost identical MaLR and ERV-L copies suggest sustained activity ${ }^{177-179}$. In contrast, rat ERV-L activity must have been silenced a few million years ago, given that the least diverged MaLR and ERV-L (MTB_Rn and MT2_Rat1) copies differ by $>4 \%$ from each other. Other class III ERVs were active earlier in rodent evolution, before the mouse-rat speciation.

In contrast to class III ERVs, class I and class II elements still thrive in rat. We reconstructed four rat-specific autonomous class I ERVs, of which two appear still active, and nine class II ERVs, of which four may still be active. The non-autonomous NICER and RAL elements represent over $60 \%$ of all rat-specific class I elements. The autonomous drivers of this group, RNNICER2 and 3, with several intact copies, are closely related to the mouse-specific MuLV. Among the potentially active autonomous class II ERVs are MYSERV_Rn, related to the Mys element in Peromyscus, and several IAP elements, one with a full-length envelope gene. The most prolific, still-active class II ERV, RNERVK3, is distantly related to the simian retroviruses and, like ERV-L and NICER, has spawned abundant non-autonomous elements characterized by closely related LTRs.

\section{Simple repeats}

Whereas the above interspersed repeats derive from transposed sequences, mammalian genomes also contain interspersed simple sequence repeats (SSRs), regions of tandemly repeated short (1-6 bp) units that probably arise from slippage during DNA replication and can expand and compress by unequal crossing

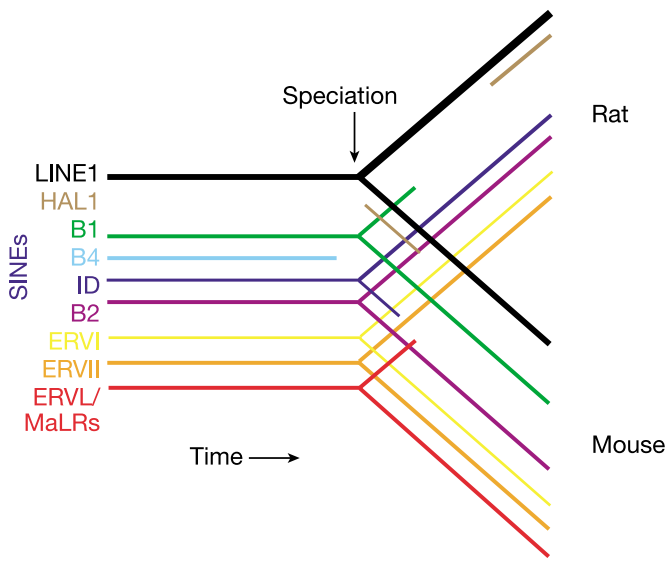

Figure 12 Historical view of rodent repeated sequences. Relationships of the major families of interspersed repeats (Table 7) are shown for the rat and mouse genomes, indicating losses and gains of repeat families after speciation. The lines indicate activity as a function of time. Note that HAL1-like elements appear to have arisen in both the mouse and rat lineages. over. Remarkable differences were noted between the SSR contents of the human and mouse genomes ${ }^{3}$. Three times as many base pairs are contained in near $(>90 \%)$ perfect SSRs in mouse than in human, and a 4-5-fold excess was revealed when excluding SSRs contained in or seeded by interspersed repeats (primarily SSRs derived from the poly A or simple repeat tails of SINEs and LINEs). SSRs are both more frequent and on average longer in mouse. Polypurine (or polypyrimidine) repeats are especially (tenfold) over-represented in the mouse genome. As discussed above, this contrasts sharply with the greater frequency of triplet repeats coding for amino acids in human than in the rodents.

Rat and mouse SSR contents show, perhaps not surprisingly, much smaller differences. They represent almost the same amount of the rat and mouse genomes (for $>90 \%$ perfect elements, $\sim 1.4 \%$ compared with $0.45 \%$ in human) and are of similar average length; for example, the average $>90 \%$ perfect $(\mathrm{CA})_{n}$ repeat, the most common SSR in mammals, is $42 \mathrm{bp}$ long in mouse and $44 \mathrm{bp}$ in rat. Some potentially significant differences are that polypurine SSRs are of similar average length but are 1.2-fold more common in mouse, whereas the rare SSRs containing CG dimers are 1.5-fold more frequently observed in rat.

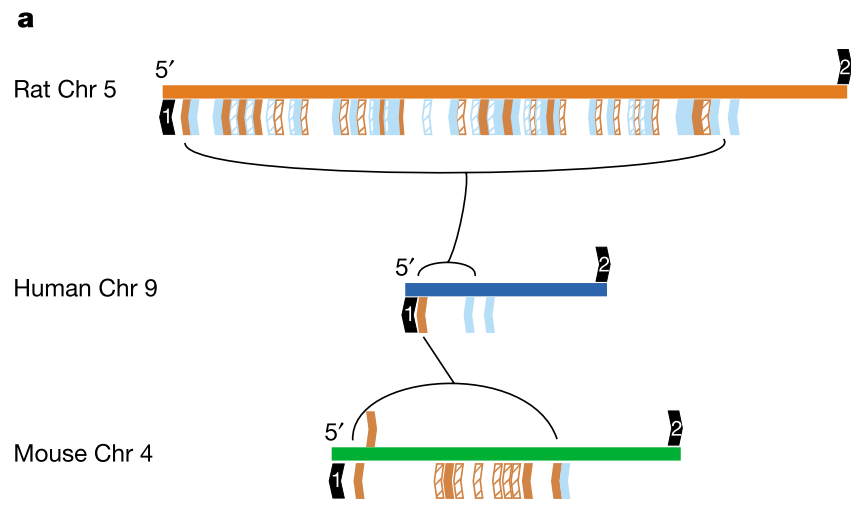

b

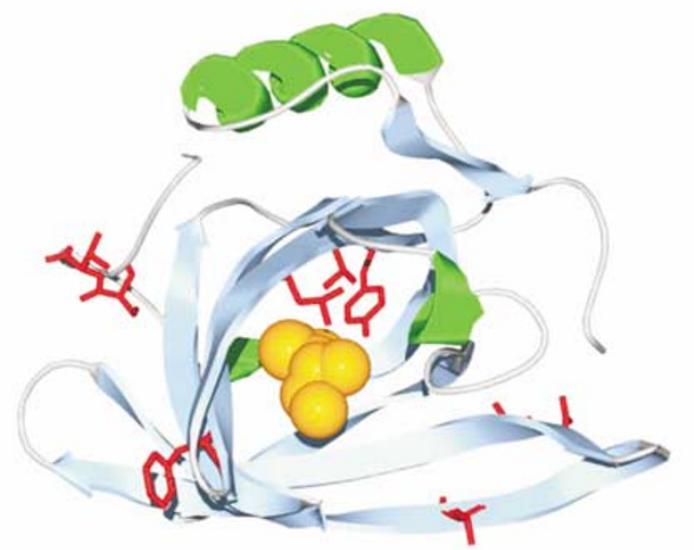

Figure 13 Adaptive remodelling of genomes and genes. a, Orthologous regions of rat, human and mouse genomes encoding pheromone-carrier proteins of the lipocalin family ( $\alpha_{2 u}$-globulins in rat and major urinary proteins in mouse) shown in brown. Zfp37-like zinc finger genes are shown in blue. Filled arrows represent likely genes, whereas striped arrows represent likely pseudogenes. Gene expansions are bracketed. Arrowhead orientation represents transcriptional direction. Flanking genes 1 and 2 are TSCOT and CTR1, respectively. $\mathbf{b}$, Site-specific $K_{A} / K_{S}$ analysis of rat $\alpha_{2 u}$-globulins. Shown in red are side-chains from codons subject to positive selection. These have been mapped to a ribbon representation of the crystal structure of rat $\alpha_{2 u}$-globulin chain $A$. 
a

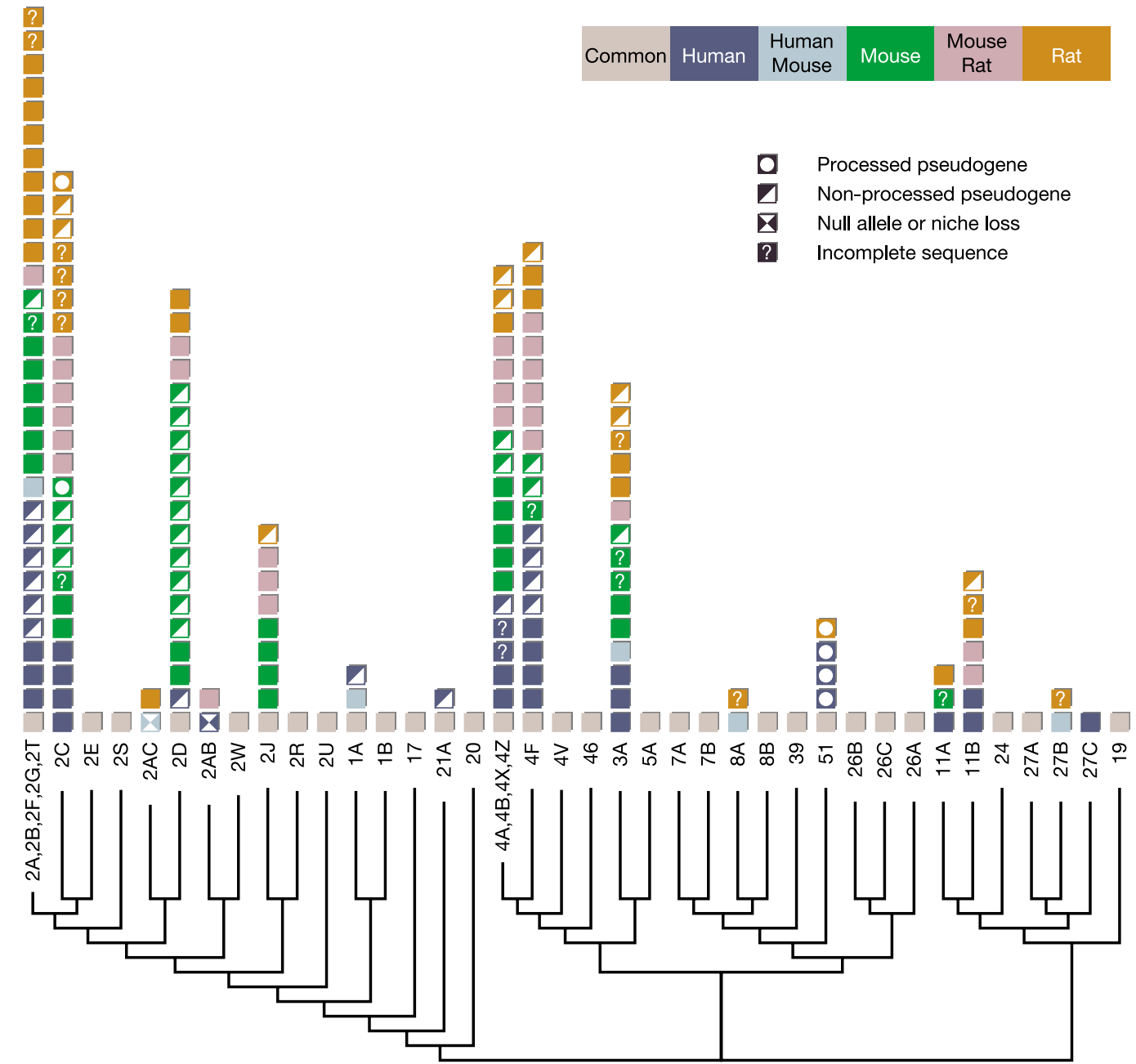

b

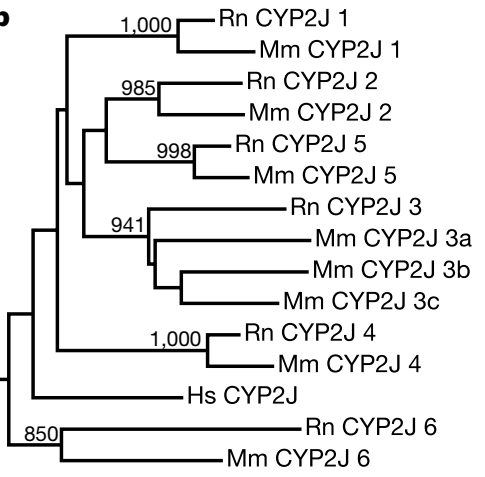

c

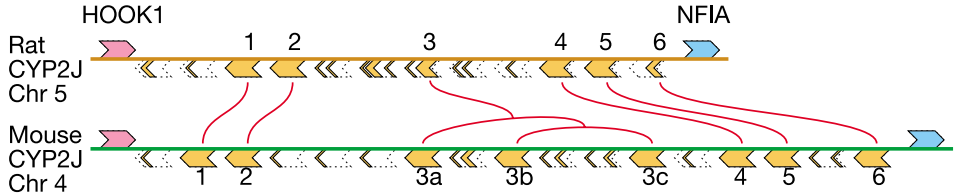

Human $\sum \geq \sum$

Chr 1
Figure 14 Evolution of cytochrome P450 (CYP) protein families in rat, mouse and human. a, Dendrogram topology from 234 full-length sequences. 279 sequences of $\geq 300$ amino acids; subfamily names and chromosome numbers are shown. Black branches have $>70 \%$ bootstrap support. Incomplete sequences (they contain Ns) are included in counts of functional genes ( 84 rat, 87 mouse and 57 human) and pseudogenes (including fragments not shown; 77 rat, 121 mouse and 52 human). 64 rat genes and 12 pseudogenes were in predicted gene sets. Human CYP4F is a null allele owing to an in-frame STOP codon in the genome, although a full-length translation exists (SwissProt P98187). Rat CYP27B, missing in the genome, is 'incomplete' because there is a RefSeq entry (NP_446215). Grouped subfamilies CYP2A, 2B, 2F, 2G, 2T and CYP4A, 4B, 4X, 4Z, occur in gene clusters; thus nine loci contain multiple functional genes in a species. One (CYP1A) has fewer rat genes than human, seven have more rodent than human, and all nine differ in rodent copy numbers. CYP2AC is a rat-specific subfamily (orthologues are pseudogenes). CYP27C has no rodent counterpart. Rodent-specific expansion, rat CYP2J, is illustrated below. $\mathbf{b}$. The neighbour-joining tree ${ }^{224}$, with the single human gene, contains clear mouse (Mm) and rat (Rn) orthologous pairs (bootstrap values $>700 / 1,000$ trials shown). Bar indicates 0.1 substitutions per site. c, All rat genes have a single mouse counterpart except for CYP2 $\mathrm{J} 3$, which has further expanded in mouse (mouse CYP2 $\mathrm{J} 3 \mathrm{a}$, $3 \mathrm{~b}$ and $3 \mathrm{c}$ ) by two consecutive single duplications. The genes flanking the CYP2J orthologous regions (rat chromosome 5, 126.9-127.3 Mb; mouse chromosome 4, 94.0-94.6 Mb; human chromosome 1, 54.7-54.8 Mb) are hook1 (H0OK1; pink) and nuclear factor I/A (NFIA; cyan). Genes (solid) and gene fragments (dashed boxes) are shown above (forward strand) and below (reverse strand) the horizontal line. No orthology relation could be concluded for most of these cases. 


\section{articles}

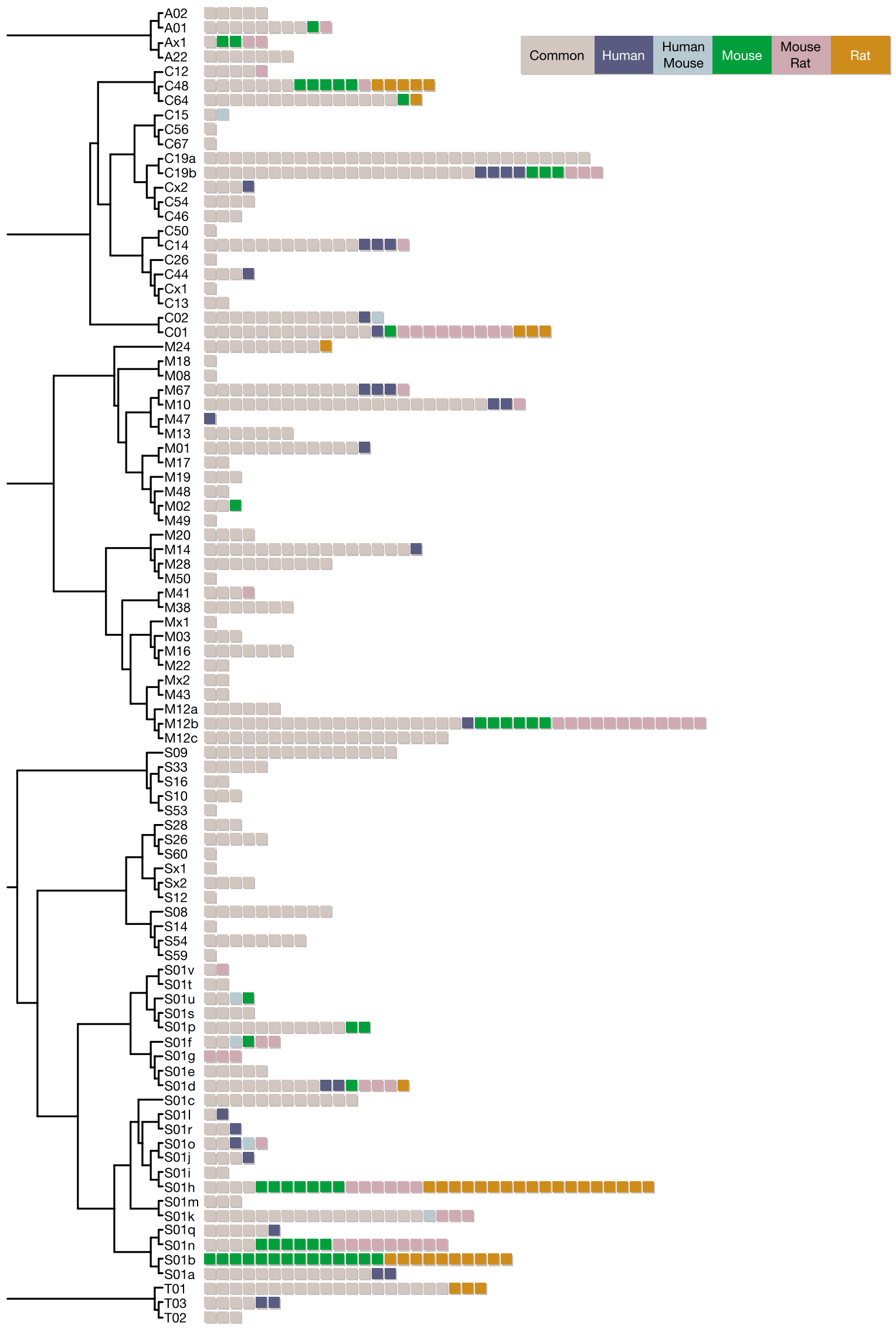

Figure 15 Comparative analysis of rat, mouse and human proteases. The complete non-

coloured according to its presence or absence in rat, mouse and human as indicated in redundant set of proteases and protease homologues from each species is distributed in five catalytic classes and 67 families. Each square represents a single protease, and is 


\section{Prevalent, medium-length duplications in rodents}

In addition to the transpositionally derived interspersed repeats and simple repeats detected by RepeatMasker and Tandem Repeat Finder, the rat and mouse genomes contain a substantial amount of medium-length unclassified duplications (typically 100$5,000 \mathrm{bp}$ ). These are readily seen in self-comparisons and in intrarodent comparisons after masking the known repeats, but they are substantially less prevalent in comparisons with the human genome (Supplementary Information). Clearly, a substantial fraction of the rodent genomes consists of currently unexplained repeats and a full characterization awaits further studies. The unclassified duplications may include: (1) novel families of low-copy rodent interspersed repeats; (2) extensions of known but not fully characterized rodent repeats; and (3) duplications generated by a mechanism different from transposition.

\section{Rat-specific biology}

A principal ambition of the RGSP was to reveal genetic differences between rats and mice that might specify their differences in physiology and behaviour. This view was well supported by the current draft sequence and predicted gene set. In particular, recently duplicated genes are enriched in elements involved in chemosensation and functional aspects of reproduction (Table 5). Here we illustrate the differences in the gene complements of rat and mouse by in-depth analyses of olfactory receptors (ORs), pheromones, cytochromes $\mathrm{P} 450$, proteases and protease inhibitors.

\section{Chemosensation}

The ability to emit and sense specific smells is a key feature of survival for most animals in the wild. Another paper ${ }^{180}$ describes the evolution of rat and mouse pheromones, vomeronasal receptors, and ORs whose genes were duplicated frequently during the time since the common ancestor of rats and mice (Table 5). Their study yielded over 200 aligned codons predicted to have been subject to adaptive evolution. They attribute the rapid evolution of these genes to conspecific competition-in particular, sexual selection.

Using a homology-based identification procedure with manual curation $^{181}$, we found 1,866 ORs in 113 locations in the rat genome: 69 multi-gene clusters and 44 single genes. After adjusting for missing sequences (the assembly covers $90.2 \%$ of the genome), we extrapolate that there are $\sim 2,070$ OR genes and pseudogenes. The rat therefore has $\sim 37 \%$ more OR genes and pseudogenes than the $\sim 1,510$ ORs of the mouse ${ }^{181,182}$, assuming similar representation of recently duplicated sequences in the two genome assemblies used. Of the 1,774 OR sequences that are not interrupted by assembly gaps, 1,227 (69\%) encode intact proteins, while the remaining 547 $(31 \%)$ sequences are probably pseudogenes with in-frame stop codons, frameshifts, and/or interspersed repeat elements. Fewer mouse OR homologues are pseudogenes $(\sim 20 \%)^{181,182}$, but the larger family size in rat still leaves it with substantially more intact ORs than the mouse $(\sim 1,430$ versus $\sim 1,210)$. Striking rat-specific expansions of two ancestral clusters account for much of the difference in OR family size and pseudogene content between rat and mouse, although many other clusters exhibit more subtle changes (not shown). Significant differences between human and mouse OR families have also been reported ${ }^{181-183}$, but the functional implications of OR repertoire size on the ability of different species to detect and discriminate odorants are not yet known.

\section{$\alpha_{2 u}$-globulin pheromones}

The $\alpha_{2 \mathrm{u}}$-globulin genes are odorant-binding proteins that also contribute to essential survival functions in animals. $\alpha_{2 \mathrm{u}}$-globulin homologues are likely to be highly heterogeneous among murid species. Several homologues (major urinary proteins) sequenced from the $\mathrm{BALB} / \mathrm{c}$ mouse are distinct from their $\mathrm{C} 57 \mathrm{BL} / 6 \mathrm{~J}$ mouse counterparts, and these also appear to be arranged differently along its genome ${ }^{184}$. Moreover, two full-length genes from other mouse strains $^{185}$ differ from their C57BL/6J orthologues-either lacking two of the bases or retaining 20 of the bases that render the C57BL/6J sequences likely to be pseudogenes (not shown).

The evolution of $\alpha_{2 \mathrm{u}}$-globulin genes on rat chromosome 5 has clearly driven a significant 'remodelling' of this genomic region (Fig. 13a). The orthologous human genomic region contains a single homologue, suggesting that the common ancestor of rodents and human possessed one gene. The genome of C57BL/6J mice contains four homologous genes, and seven pseudogenes, whereas the rat genome contains ten $\alpha_{2 \mathrm{u}}$-globulin genes and 12 pseudogenes in a single region (Fig. 13a).

Phylogenetic trees constructed using amino acid, and non-coding DNA, sequences show that, surprisingly, the rat $\alpha_{2 u}$-globulin gene clusters appear to have arisen recently via a rapid burst of gene duplication since the rat-mouse split (Table 5; data not shown). This is consistent with the Rfp37-like zinc-finger-like pseudogene having uniquely 'hitchhiked' for virtually all of the rat-specific $\alpha_{2 u}$-globulin gene duplications (Fig. 13a). The sequences of these genes are also evolving rapidly, with median $K_{\mathrm{A}} / K_{\mathrm{S}}$ values of 0.77 and 1.06 for rat and mouse genes, respectively. Amino acid sites that appear to have been subject to adaptive evolution are situated both within the ligand-binding cavity, and on the solvent-exposed periphery of the $\alpha_{2 \mathrm{u}}$-globulin structure ${ }^{139}$ (Fig. 13b). This demonstrates how genome analysis can reveal the imprint of adaptive evolution from megabase to single-base levels.

The rapid evolution of these genes, and the remodelling of their genomic regions, can be attributed to the known roles of rat $\alpha_{2 u}$-globulins and mouse major urinary proteins in conspecific competition and sexual selection. These proteins are pheromones and pheromone carriers that are present in large quantities in rodent urine, and act as scent markers indicating dominance and subspecies identity ${ }^{186,187}$.

\section{Detoxification}

Cytochrome P450 is a well-recognized participant in metabolic detoxification, and we also observe rapid evolution within this family. These enzymes metabolize a large number of toxic and endogenous compounds ${ }^{188}$ and thus are particularly relevant to clinical and pharmacological studies in humans. As rodents are important model organisms for understanding human drug metabolism, it is important to identify $1: 1$ orthologues and species-specific expansions and losses ${ }^{189}$. Compared with human genes, there are clear expansions of several rodent $\mathrm{P} 450$ subfamilies, but there are also significant differences between rat and mouse subfamilies (Fig. 14a). The fastest-evolving subfamily seems to be CYP2J, containing a single gene in human, but at least four in rat and eight in mouse (Fig. 14b, c). CYP2J enzymes catalyse the NADPH-dependent oxidation of arachidonic acid to various eicosanoids, which in turn possess numerous biological activities including modulation of ion transport, control of bronchial and vascular smooth muscle tone, and stimulation of peptide hormone secretion ${ }^{190}$. The genomic ordering of genes and their phylogenetic tree indicate an ongoing expansion in the rodents (Fig. 14b, c). This suggests that adaptive evolution has been involved in diversifying their functions. Moreover, detailed study of the nuclear receptors, a highly conserved family of transcription factors, revealed that PXR and CAR, two nuclear receptors regulating CYP genes involved with detoxification ${ }^{191}$, have the two highest nucleotide substitution rates in their ligand binding domains, whereas SF-1, the nuclear receptor regulating CYP19 (ref. 192), which has not undergone expansion, is more conserved, like other nuclear receptors ${ }^{193}$.

\section{Proteolysis}

Protease and protease inhibitor genes also represent an example of rapid evolution in the rat genome. Proteases are a structurally and functionally heterogeneous group of enzymes involved in multiple biological and pathological processes ${ }^{194}$. The rat contains 626 
protease genes, $\sim 1.7 \%$ of the rat gene count ${ }^{124}$, more than human (561) but similar to mouse $(641)^{125}$. Of the rat protease genes, 102 are absent from human, and 42 are absent from mouse (Fig. 15). Several rat gene families have expanded, including placental cathepsins, testases, kallikreins and haematopoietic serine proteases; others appear to have formed pseudogenes in humans (Table 8). These protease families are mainly involved in reproductive or immunological functions, and have evolved independently in the rat and mouse lineages.

The rat protease inhibitor complement contains 183 members, similar to mouse (199) but larger than human (156). As with the protease genes, the rapid evolution in protease inhibitors derives from differential expansions of specific families such as serpins and cystatins. The concomitant expansions in rat and mouse proteases and their inhibitors appear to reflect homeostasis of protein turnover.

These gene family expansions dramatically illustrate how largescale genomic changes have accompanied species-specific innovation. Positive selection of duplicated genes has afforded the rat an enhanced repertoire of precisely those genes that allow reproductive success despite severe competition from both within its own, and with other, species. This serves as a general illustration of the importance of chemosensation, detoxification and proteolysis in innovation and adaptation.

\section{Human disease gene orthologues in the rat genome}

A further strong motivation for sequencing the rat genome was to enhance its utility in biomedical research. Although the rat is already recognized as the premier model for studying the physiological aspects of many human diseases, it has not had as prominent a role in the study of simple genetic disease traits. As more than 1,000 human mendelian disorders now have associated loci and alleles, there is now a tremendous opportunity to link the new knowledge of the rat genome with data from the human disease examples. The precise identification of the rat orthologues of human genes that are mutated in disease creates further opportunities to discover and develop rat models.

Predicted rat genes were compared with 1,112 well-characterized human disease genes ${ }^{195}$ that were verified and classified on the basis of pathophysiology (H.H., E.E.W., H.W., K.G.W., H.X., L.G., P.D.S., D.N.C., D.S., M.M.A., C.P.P. and K.F., unpublished work). As predicted by Ensembl, 844 (76\%) have 1:1 orthologues in the rat. These predictions are likely to be of high quality because $97.4 \%$ of the 11,422 rat:human 1:1 orthologues predicted by Ensembl were found in orthologous genomic regions.

We asked if these 'disease orthologue' pairs were distinguishable from other rat-human orthologues. Ensembl automatically predicts that 11,522 human genes have rat 1:1 orthologues (corresponding to $46 \%$ of all Ensembl predicted human genes). By contrast, a much higher proportion $(76 \%)$ of human disease genes have Ensembl-predicted rat 1:1 orthologues. Careful analysis of the remaining 268 human genes that were not predicted by Ensembl to show 1:1 orthology indicated that only six of the human disease genes lack likely rat orthologues among genome, cDNA, EST and protein sequences ${ }^{196}$. Thus, it appears that, in general, genes involved in human disease are unlikely to have diverged, or to have become duplicated, deleted or lost as pseudogenes, between rat and human (conservation of orthologues discussed above).

We next compared $K_{\mathrm{S}}, K_{\mathrm{A}}$ and the $K_{\mathrm{A}} / K_{\mathrm{S}}$ ratio values of 'disease orthologues' with those of all remaining orthologue pairs. Only the $K_{\mathrm{S}}$ distributions differed significantly ${ }^{196}$, suggesting that coding regions of human disease genes and their rat counterparts have mutated more rapidly than the non-disease genes. This might result from factors influencing the specific loci, or the disease genes may characteristically reside in genomic regions that exhibit higher mutation rates.

The disease gene set was next grouped into 16 disease-system categories and analysed using a non-parametric test for $K_{\mathrm{A}} / K_{\mathrm{S}}$ (human/rat) ${ }^{196}$ (Fig. 16). Only five disease systems exhibited significant $K_{\mathrm{A}} / K_{\mathrm{S}}$ differences with respect to the remaining samples $(P<0.05)$. Neurological and malformation-syndrome disease categories manifested the lowest median $K_{\mathrm{A}} / K_{\mathrm{S}}$ ratios that are consistent with purifying selection acting on these gene sets. With a comparison of the mean to the mean and standard deviation of the null hypothesis, [(Mean-Mean 0$) /$ Std0] of $-4.63(P<0.0001)$, the neurological disease gene set revealed the most evidence for purifying selection of the disease gene categories examined. In contrast, the pulmonary, haematological and immune categories manifested the highest median $K_{\mathrm{A}} / K_{\mathrm{S}}$ ratios, and the genes of the immune system disease category, with a value for (Mean-Mean0)/ Std0 of $4.98(P<0.0001)$, show the highest $K_{\mathrm{A}} / K_{\mathrm{S}}$ ratios. These results are consistent with a role for more positive selection, or reduced selective constraints, among these genes.

Where possible, we further considered conservation of these pathophysiology-based gene sets among orthologues of more diverse phyla, including mouse, fish, fly, nematode worm and

\begin{tabular}{|c|c|c|c|c|}
\hline Protease & Rat gene / locus & Human gene / locus & Mouse gene / locus & Function \\
\hline Absent genes in assembly & 13 from $626(2.07 \%)$ & 5 from 561 (0.89\%) & 5 from 641 (0.78\%) & \\
\hline \multicolumn{5}{|l|}{ Expanded families } \\
\hline Placental cathepsins & 10 genes / 17p14 & Absent & 8 genes / 13B3 & Reproduction \\
\hline Testins & 3 genes / 17p14 & Absent & 3 genes / 13B3 & Reproduction \\
\hline Glandular kallikreins & 10 genes / 1q21 & Absent & 15 genes / 7B2 & Reproduction \\
\hline Mast cell chymases/granzymes & 28 genes / 15p13 & 4 genes / 14q11 & 17 genes / 14C1 & Host defence \\
\hline \multicolumn{5}{|l|}{ Human pseudogenes } \\
\hline Chymosin & 1 gene / 2q34 & $1 \mathrm{ps} / 1 \mathrm{p} 13$ & 1 gene / 3F3 & Digestion \\
\hline Distal intestinal serine proteases & 2 genes / 10q12 & $1 \mathrm{ps} / 16 \mathrm{p} 12$ & 2 genes / 17A3 & Digestion \\
\hline Pancreatic elastase & 1 gene / 7 q35 & $1 \mathrm{ps} / 12 \mathrm{q} 13$ & 1 gene / 15F3 & Digestion \\
\hline Fertilins and reproductive ADAMs & 7 genes / various loci & $6 \mathrm{ps} /$ various loci & 8 genes / various loci & Reproduction \\
\hline Testases & 4 genes / 16q12 & 3 ps / 8p22 & 9 genes / 8B1 & Reproduction \\
\hline Testis serine proteases & 5 genes / various loci & $5 \mathrm{ps} /$ various loci & 6 genes / various loci & Reproduction \\
\hline Implantation serine proteases & 2 genes / 10q12 & $1 \mathrm{ps} / 16 \mathrm{p} 13$ & 2 genes / 17A3 & Reproduction \\
\hline Airway trypsin-like proteases & 3 genes / 14p21 & $3 p s / 4 q 13$ & 3 genes / 5E1 & Host defence \\
\hline \multicolumn{5}{|l|}{ Rat pseudogenes } \\
\hline Calpain 13 & $1 \mathrm{ps} / 6 \mathrm{q} 12$ & 1 gene / 2p23 & 1 gene / 17E2 & Reproduction? \\
\hline Pyroglutamyl-peptidase II & $1 \mathrm{ps} / 1 \mathrm{q} 22$ & 1 gene / 15q26 & 1 gene / 7C & Metabolism \\
\hline Gln-fructose- 6 -P transamidase 3 & $1 \mathrm{ps} / \mathrm{Xq} 14$ & 1 gene / Xq21 & $1 \mathrm{ps} / \mathrm{XC3}$ & Metabolism \\
\hline Aminopeptidase MAMS/L-RAP & $1 \mathrm{ps} / 1 \mathrm{q} 12$ & 1 gene / 5q15 & $1 \mathrm{ps} / 17 \mathrm{~A} 3$ & Host defence \\
\hline Carboxypeptidase $\mathrm{O}$ & $1 \mathrm{ps} / 9 \mathrm{q} 31$ & 1 gene / 2q33 & $1 \mathrm{ps} / 1 \mathrm{C} 2$ & Unknown \\
\hline Procollagen III N-endopeptidase & $1 p s / 19 q 12$ & 1 gene / 16q24 & $1 \mathrm{ps} / 8 \mathrm{E} 2$ & Metabolism ? \\
\hline Kallikrein-2 and -3 & $2 p s / 1 q 21$ & 2 genes / 19q13 & $1 \mathrm{ps} / 7 \mathrm{~B} 2$ & Reproduction \\
\hline Testis-specific protein 50 & $1 \mathrm{ps} / 8 \mathrm{q} 32$ & 1 gene / 3p21 & 1 gene / 9F2 & Reproduction \\
\hline
\end{tabular}


yeast orthologues. Overall, we obtained results consistent with those reported here for these rat:human 1:1 orthologous gene disease categories ${ }^{196}$. These results demonstrate that the individual genes that constitute various disease systems exhibit significantly different average evolutionary rates. The higher evolutionary rates noted for the immune system disease genes are consistent with a previous finding that lymphocyte-specific genes evolve relatively rapidly ${ }^{197}$ and may indicate rapid diversification of the functions of the immune systems of rodents and humans. This is expected for genes involved in controlling species-restricted infectious agents if strong adaptive pressure acts during host-pathogen co-evolution. Thus, the results of studies of these rodent genes may be less directly relevant to our understanding of human immune system diseases than results obtained for other pathophysiology disease systems where conservation is greater and purifying selection is stronger.

We have also specifically examined a number of genes that harbour triplet nucleotide repeats, and are involved in human neurological disorders such as Huntington's disease, a condition known to be caused by CAG triplet repeat expansion producing abnormally long polyglutamine tracts in an otherwise normal protein $^{198}$. Analysis of the rat-human orthologues of these disease genes indicated that repeat-expansion disease genes exhibit a repeat length that is substantially shorter in the rat than that found in the normal human gene (Fig. 17). In all cases, human disease genes localize below the line demarcating 1:1 length correlation, showing that rat orthologues uniformly bear shorter repeats. At present, there are no naturally occurring rat strains described that exhibit neurological disease associated with repeat-expansion mechanisms. The shorter repeat length of these orthologues in the rat would be consistent with either the lack of repeat-expansion mutational mechanisms in the rat or the failure of these orthologues to achieve a 'critical repeat length' susceptible to such mutational mechanisms. Other human genes, not at present known to be associated with disease, also contain glutamine repeats that are much shorter in the rat orthologues, and thus, could be investigated as potential disease candidates ${ }^{196}$. These triplet-repeat-bearing genes may be susceptible to mutations that arise through repeat-expansion mechanisms. In Fig. 17, it may also be observed that a relatively high proportion of repeats are significantly longer in the rat than in their corresponding human orthologue.

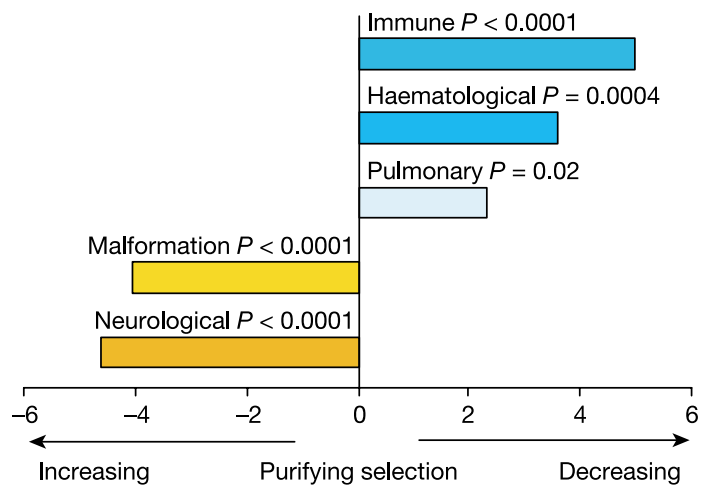

Figure 16 Selective constraints differ for human disease systems in the rat genome. Human disease system categories showing significant differences $(P<0.05)$ in a nonparametric test (Mann-Whitney-Wilcoxon) comparing $K_{\mathrm{A}} / K_{\mathrm{S}}$ (human:rat) ratios. $P$ values from two-level tests between genes from one disease system and the remaining genes. (Mean-Mean0)/Std0 values from multi-level tests from 16 categorized disease systems. Negative values (shown in yellow and orange) for neurological $(-4.63)$ and malformationsyndrome $(-4.04)$ categories were observed to be consistent with $K_{\mathrm{A}} / K_{\mathrm{S}}$ ranges in which purifying selection predominates. Immune, haematological and pulmonary categories show positive values of 4.98, 3.59 and 2.34 , respectively (for complete data set and details, see ref. 199).
In addition to enabling the direct comparison of rat-human disease orthologues, the rat genome sequence itself is an invaluable aid for the discovery of additional rat genes that can be studied as disease models. Two general modes can now be pursued. First, genes underlying disease phenotypes with simple inheritance that have been mapped to chromosomal regions can be more easily pursued in both species. Indeed, the rearrangements of conserved segments between the two species in this map were found to have significant value, because they tighten the boundaries of the mapped disease regions and thus reduce the number of genes that could potentially be associated with a given disease phenotype ${ }^{113}$. Second, the identification of multiple alleles contributing to quantitative and complex trait differences that are involved in disease processes can be pursued with more accuracy, both in the initial association phases, and in subsequent efforts to detect causative alleles.

\section{Rat single nucleotide polymorphisms}

The discovery and cataloguing of the natural DNA variation that persists between individual rat strains will allow further research using rat model systems. Although many rat microsatellites have been characterized and studied, single nucleotide polymorphisms (SNPs) are of more general interest because of their probable ubiquity, and the ease with which they can be assayed. SNP data have three broad applications: (1) the individual markers can be used in ongoing efforts to associate phenotypes that have complex underlying genetic components, with specific sites in the genome. (2) A panel of such markers can be used in conjunction with selective breeding and chromosome mechanics, to generate rat strains that are amenable to the kinds of manipulations that will hasten the discovery of important alleles. (3) A set of such markers can be used to detail the history of the different genomic events that have led to the structure of the genomes of contemporary rat strains. A detailed map of these events has a utility analogous to the current human haplotype (HapMap) mapping project ${ }^{199}$ and will probably

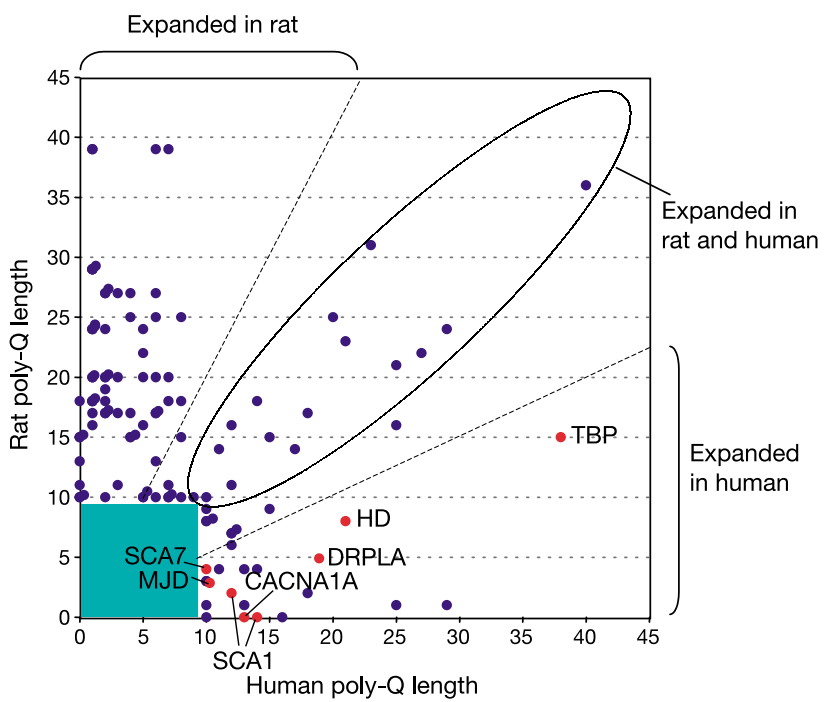

Figure 17 Polyglutamine repeat length comparison between human and rat. Points represent protein poly- $Q$ length for rat and human. Red points correspond to repeats in genes associated with human disease: SCA1, spinocerebellar ataxia 1 protein, or ataxin1; SCA7, spinocerebellar ataxia 7 protein; MJD, Machado-Joseph disease protein; CACNA1A, spinocerebellar ataxia 6 protein, or calcium channel alpha 1 A subunit isoform 1; DRPLA, dentatorubral pallidoluysian atrophy protein; HD, Huntington's disease protein, or huntingtin; TBP, TATA binding protein or spinocerebellar ataxia 17 protein. Repeat lengths over ten were examined; green shading delineates the range not included in our analysis. Also noted are a set that are expanded in rat and human (black circle) and a set where repeats are expanded in the rat. 
aid disease gene identification, as recently suggested for the mouse $^{200}$.

The Rnor3.1 draft sequence was generated primarily from DNA of a single inbred rat line. This maximized the likelihood of deriving an accurate sequence assembly, but reduced any likely discovery of natural variation in this phase of the project. As a consequence there has been no large-scale public SNP discovery from rat genomic sequencing. A pilot project based on coding (c)SNP discovery has been initiated, however ${ }^{201}$, as these cSNPs represent a particularly important subset of variants that may have direct functional significance ${ }^{202}$. These data have illustrated both immediate applications and the long-term potential for an effort aimed at comprehensive SNP discovery.

\section{Conclusions}

As the third mammalian genome to be sequenced, the rat genome has provided both predictable and surprising information about mammalian species. Although it was clear at the outset of this programme that ongoing rat research would benefit from the resource of a genome sequence, there was uncertainty about how many new insights would be found, especially considering the superficial similarities between the rat and the already sequenced mouse. Instead, the results of the sequencing and analysis have generated some deep insights into the evolutionary processes that have given rise to these different species. In addition, the project has been invaluable in further developing the methods for the generation and analysis of large genome sequence data sets.

The generation of the rat draft tested the new 'combined approach' for large genome sequencing. As the overall assembly is of high quality, there is no doubt that this overall strategy, and the supporting software we have developed, provides a suitable approach for this problem. Because we included a BAC 'skimming' component in the underlying data set, the assembly recovered a fraction of the genome that was expected, by analogy to the mouse project, to be difficult to assemble from pure WGS data. In addition, the BAC skimming component allowed progressive generation of high-quality local assemblies that were of use to the rat research community as the project developed. On the other hand, although the $\mathrm{BAC}$ component used here was far less expensive than the fully ordered and highly redundant set used in the hierarchical approach to sequencing the human genome, it nevertheless increased the overall cost of data production relative to a WGS approach.

The issue of efficacy of WGS versus other approaches to the sequencing of large genomes remains a matter of earnest scientific debate. In ongoing projects at different centres that participated in the RGSP consortium, different approaches are being used to tackle new genomes. These include pure WGS methods, the combined approach and variations on that methodology. The future application of the different procedures depends on the target genome sizes, the expected degree of heterogeneity (that is, polymorphism) in the organism to be sequenced, and the preferences of the individual centre. So far, all the genomes that have been analysed by RGSP consortium members have been of high quality and we anticipate that this will continue as the benefits and disadvantages of different approaches are further studied and analysed.

The rat genome data have improved the utility of the rat model enormously. Now that near-complete knowledge of the rat gene content is realizable, individual researchers have a data source for the rat 'parts list' that can be explored with the high degree of confidence and precision that is appropriate for biomedical research. A similar improvement has been made in the resources for physical and genetic mapping, because the relative position of individual markers is now known with high confidence and there are now computational resources to bridge the process of genetic association with gene modelling and experimental investigation. These advances have been reflected by measured increases in the use of all the rat-specific public genome data sets that can be accessed online, as well as by the informally assessed increases in overall 'genomic' research of this model.

The expected benefit of a third mammalian sequence providing an outgroup by which to discriminate the timing of events that had already been noted between mouse and human was fully realized. Using the three sequences and other partial data sets from additional organisms, it was possible to measure some of the overall faster rate of evolutionary change in the rodent lineage shared by mice and rats, as well as the peculiar acceleration of some aspects of rat-specific evolution. The observation of specific expanded gene families in the rat should provide material for targeted studies for some time.

At this time there is no plan to further upgrade or finish the rat genome sequence. This programme decision is a consequence of the high cost of converting draft sequence to finished data, and the pressing need to analyse new genomes. However, as the distant objective of very-low-cost sequencing or other advances that can improve draft sequences inexpensively are realized, it might be envisioned that a rat sequence that approaches the quality of the current human data will be produced. A finished rat genome may answer many questions, as specific clues already show that areas of the genome that are most difficult to resolve in a random sequencing project are also those areas that are most dynamic, and therefore of high potential interest in an evolutionary context.

Despite the advances represented here, we are clearly still at the beginning of the full analysis of the mammalian genome and its complex evolutionary history. Much of the additional data that are required to complete this story will be from other genomes, distantly related to rat. Nevertheless, a considerable body of data remains to be developed from this species. In addition to the distant prospect of a finished rat genome, analysis of other rat strains may yield genome-wide polymorphism data, while targeted efforts to generate cDNA clone collections will provide rat-specific reagents for routine use in research. Together with the ongoing efforts to fully develop methods to genetically manipulate whole rats and provide effective 'gene knockouts', the current and future rat genome resources will ensure a place for this organism in genomic and biomedical research for some time.

\section{Methods}

\section{DNA sequencing and data access}

Paired-end reads from BAC and WGS libraries were produced as previously described ${ }^{2,203}$ Unprocessed sequence reads are available from the NCBI Trace Archive (ftp:// ftp.ncbi.nih.gov/pub/TraceDB/rattus_norvegicus/); raw eBAC assembly data are available from the BCM-HGSC (http://www.hgsc.bcm.tmc.edu/Rat/); and the released Rnor3.1 assembly is available from the BCM-HGSC (ftp://ftp.hgsc.bcm.tmc.edu/pub/analysis/rat/ ), the NCBI (ftp://ftp.ncbi.nih.gov/genomes/R_norvegicus), and the UCSC (http:// genome.ucsc.edu/downloads.html).

\section{Genome assembly}

Assembly of the rat genome by the Atlas system is described in detail elsewhere ${ }^{54}$. Earlier assemblies (Rnor2.0/2.1) of the initial data set were based on 40 million total reads and $19,000 \mathrm{BAC}$ skims. These assemblies spanned $2.66 \mathrm{~Gb}$ and comprised over 900 ultrabactigs with $N_{50}$ of over $5 \mathrm{Mb}$. They differed only in the removal of short artefactual duplications from Rnor2.0. Rnor3.1 includes another 1,100 BACs, selected to fill gaps in Rnor2.1. Because of the comprehensive coverage of the genome by Rnor2.0/2.1, it was used for the initial predictions of genes and proteins.

\section{BAC fingerprints}

An agarose-gel-based fingerprinting methodology ${ }^{204-207}$ was employed to generate HindIII fingerprints from 199,782 clones in the CHORI-230 BAC library. The contig assembly was subjected to manual review and editing to refine clone order within contigs and to make merges between contigs, using tools provided in the FPC software ${ }^{208-210}$. Fingerprints for 5,250 RPCI-31 PACs ${ }^{211}$ and RPCI-32 BACs were subsequently added to allow correlation between the fingerprint map and a developing YAC map of the rat genome. BAC and PAC clones are available through BACPAC Resources at CHORI (bacpacorders@chori.org).

\section{BAC, PAC and YAC maps}

Markers generated from BAC and PAC clones were hybridized against YAC ${ }^{58}$ (R.D., Pmatch, unpublished software) and radiation hybrid libraries ${ }^{61,212}$ to produce independent maps that were subsequently combined. Genetic markers from two rat 
genetic maps ${ }^{61}$ and the radiation hybrid map ${ }^{59}$ were aligned to the Rnor3.1 assembly using BLAT ${ }^{123}$ (when sequence was available) or electronic polymerase chain reaction $(\mathrm{EPCR})^{213}$

\section{Finished sequence used for quality assessment of the assembly}

To assess the accuracy of the Atlas assembly, the Rnor 3.1 sequence was compared to $13 \mathrm{Mb}$ of sequences that had been finished to high quality.

\section{Large-scale rearrangements}

We compared these assemblies: Human (April 2003, NCBI build 33); Mouse (February 2003, NCBI build 30); and Rat (June 2003, Rnor3.1). Repeats were masked using RepeatMasker (A.S. \& P. Green, unpublished work; see http://ftp.genome. washington.edu/RM/RepeatMasker.html) and TandemRepeatFinder ${ }^{214}$. Local alignments were produced using PatternHunter ${ }^{70}$ (Supplementary Information). Repeat contamination was removed and the remaining similarities combined into two- and threeway anchors ${ }^{73}$ and synteny blocks produced at various resolutions using GRIMMSynteny ${ }^{71}$.

\section{Genome-wide visualization of conserved synteny}

Pairwise comparisons of the genomes of human, mouse and rat using MULTIZ ${ }^{69,215}$ MLAGAN $^{216,217}$, MAVID ${ }^{110}$, PatternHunter ${ }^{70}$ and Pash ${ }^{72}$ were merged into blocks of conserved synteny ${ }^{69,71,72}$, and the 1-Mb-resolution images were displayed using the Virtual Genome Painting method (M.L.G.-G. et al., unpublished work; http:// www.genboree.org).

\section{Rat segmental duplications}

Segmental duplications $>5 \mathrm{~kb}$ were identified, extracted and aligned as described ${ }^{218}$, and paralogous sequence relationships were assessed using PARASIGHT visualization software (J.A.B., unpublished work; Supplementary Information).

\section{Venn diagram}

Pairwise and three-way alignments generated using BLASTZ ${ }^{219}$ and MULTIZ ${ }^{215}$ or $\mathrm{HUMOR}^{215}$ were analysed to classify each nucleotide in the three genomes by the species with which it aligns: in all three species, aligning between human and rat (but not mouse), between human and mouse (but not rat), or between mouse and rat (but not human). Other nucleotides are species-specific; unassigned nucleotides occupying gaps in the genome assemblies were excluded. On the basis of output from RepeatMasker ${ }^{164}$ and RepeatDater $^{89}$, nucleotides were assigned to categories (of non-repetitive, repetitive with a certain ancestry, or repetitive but unassigned) and counted. See Supplementary Table SI-1 for details.

\section{Gene prediction}

ENSEMBL transcript models were built from 28,478 rodent proteins that were aligned to the genome using a combination of Pmatch (R.D., unpublished software), BLAST ${ }^{220}$ and GeneWise $^{221}$. Models based on 5,083 vertebrate proteins were added in regions without rodent-protein-based models. UTRs were added using 11,170 transcripts built from 8,615 different rat cDNAs aligned to the genome using BLAT, with coverage $\geq 90 \%$ and identity $\geq 95 \%$. This procedure (as described ${ }^{112}$ but without GENSCAN predictions), gave rise to 18,241 genes and 20,373 transcripts. This is the protein-based gene set. Rat and mouse cDNA and rat EST-based gene sets were also built. See Supplementary Information for details.

\section{Non-processed pseudogene identification}

Human and mouse genes related by 1:1 orthology and lacking an apparent rat orthologue were considered. See Supplementary Information for details.

\section{High-resolution analyses of chromosome 10}

These were performed predominantly on the whole genome alignments ${ }^{217}$. Plots in Fig. 9 were generated by sliding windows of width $2 \mathrm{Mb}$ and a step size of $400 \mathrm{~kb}$ (total $=277$ windows). See Supplementary Information for details.

Received 31 December 2003; accepted 20 February 2004; doi:10.1038/nature02426.

1. Darwin, C. On The Origin of Species by Means of Natural Selection 1st edn, Ch. 4, 108 (John Murray, London, 1859).

2. International Human Genome Sequencing Consortium. Initial sequencing and analysis of the human genome. Nature 409, 860-921 (2001)

3. Mouse Genome Sequencing Consortium. Initial sequencing and comparative analysis of the mouse genome. Nature 420, 520-562 (2002).

4. Adkins, R. M., Gelke, E. L., Rowe, D. \& Honeycutt, R. L. Molecular phylogeny and divergence time estimates for major rodent groups: evidence from multiple genes. Mol. Biol. Evol. 18, 777-791 (2001).

5. Springer, M. S., Murphy, W. J., Eizirik, E. \& O'Brien, S. J. Placental mammal diversification and the Cretaceous-Tertiary boundary. Proc. Natl Acad. Sci. USA 100, 1056-1061 (2003).

6. Canby, T. Y. The rat, lapdog of the devil. Nat. Geogr. July, 60-87 (1977).

7. Robinson, R. Genetics of the Norway Rat (Pergamon, Oxford, 1965).

8. Barnett, S. A. The Story of Rats. Their Impact on Us, and Our Impact on Them Ch. 2, 17-18 (Allen and Unwin, Crows Nest, Australia, 2002).

9. Hedrich, H. J. in History, Strains, and Models in the Laboratory Rat (ed. Krinke, G. J.) 3-16 (Academic, San Diego, 2000).

10. Lindsey, J. R. in The Laboratory Rat (eds Baker, H. J., Lindsey, J. R. \& Weisbroth, S. H.) 1-36 (Academic, New York, 1979).

11. Greenhouse, D. D., Festing, M. F. W., Hasan, S. \& Cohen, A. L. in Genetic Monitoring of Inbred Strains of Rats (ed. Hedrich, H. J.) 410-480 (Gustav Fischer, Stuttgart, 1990).
12. Kuntz, C. et al. Comparison of laparoscopic versus conventional technique in colonic and liver resection in a tumor-bearing small animal model. Surg. Endosc. 16, 1175-1181 (2002).

13. Kitagawa, K., Hamada, Y., Nakai, K., Kato, Y. \& Okumura, T. Comparison of one- and two-step procedures in a rat model of small bowel transplantation. Transplant. Proc. 34, 1030-1032 (2002)

14. Sauve, Y., Girman, S. V., Wang, S., Keegan, D. J. \& Lund, R. D. Preservation of visual responsiveness in the superior colliculus of RCS rats after retinal pigment epithelium cell transplantation. Neuroscience 114, 389-401 (2002).

15. Wang, H. et al. Attenuation of acute xenograft rejection by short-term treatment with LF15-0195 and monoclonal antibody against $\mathrm{CD} 45 \mathrm{RB}$ in a rat-to-mouse cardiac transplantation model. Transplantation 75, 1475-1481 (2003).

16. Alves, A. et al. Total vascular exclusion of the liver enhances the efficacy of retroviral-mediated associated thymidine kinase and interleukin-2 genes transfer against multiple hepatic tumors in rats. Surgery 133, 669-677 (2003).

17. Liu, M. Y., Poellinger, L. \& Walker, C. L. Up-regulation of hypoxia-inducible factor $2 \alpha$ in renal cell carcinoma associated with loss of Tsc-2 tumor suppressor gene. Cancer Res. 63, 2675-2680 (2003).

18. Jin, X. et al. Effects of leptin on endothelial function with OB-Rb gene transfer in Zucker fatty rats. Atherosclerosis 169, 225-233 (2003).

19. Ravingerova, T., Neckar, J. \& Kolar, F. Ischemic tolerance of rat hearts in acute and chronic phases of experimental diabetes. Mol. Cell. Biochem. 249, 167-174 (2003).

20. Taylor, J. R. et al. An animal model of Tourette's syndrome. Am. J. Psychiatry 159, 657-660 (2002)

21. Smyth, M. D., Barbaro, N. M. \& Baraban, S. C. Effects of antiepileptic drugs on induced epileptiform activity in a rat model of dysplasia. Epilepsy Res. 50, 251-264 (2002).

22. McBride, W. J. \& Li, T. K. Animal models of alcoholism: neurobiology of high alcohol-drinking behavior in rodents. Crit. Rev. Neurobiol. 12, 339-369 (1998)

23. Crisci, A. R. \& Ferreira, A. L. Low-intensity pulsed ultrasound accelerates the regeneration of the sciatic nerve after neurotomy in rats. Ultrasound Med. Biol. 28, 1335-1341 (2002).

24. Ozkan, O. et al. Reinnervation of denervated muscle in a split-nerve transfer model. Ann. Plast. Surg. 49, 532-540 (2002).

25. Fray, M. J., Dickinson, R. P., Huggins, J. P. \& Occleston, N. L. A potent, selective inhibitor of matrix metalloproteinase- 3 for the topical treatment of chronic dermal ulcers. J. Med. Chem. 46, 3514-3525 (2003).

26. Petratos, P. B. et al. Full-thickness human foreskin transplantation onto nude rats as an in vivo model of acute human wound healing. Plast. Reconstr. Surg. 111, 1988-1997 (2003).

27. Hussar, P. et al. Bone healing models in rat tibia after different injuries. Ann. Chir. Gynaecol. 90, 271-279 (2001).

28. Yang, T. D., Pei, J. S., Yang, S. L., Liu, Z. Q. \& Sun, R. L. Medical prevention of space motion sickness-animal model of therapeutic effect of a new medicine on motion sickness. Adv. Space Res. 30, 751-755 (2002)

29. Forte, A. et al. Stenosis progression after surgical injury in Milan hypertensive rat carotid arteries. Cardiovasc. Res. 60, 654-663 (2003).

30. Komamura, K. et al. Differential gene expression in the rat skeletal and heart muscle in glucocorticoid-induced myopathy: analysis by microarray. Cardiovasc. Drugs Ther. 17, 303-310 (2003)

31. McBride, M. W. et al. Functional genomics in rodent models of hypertension. J. Physiol. (Lond.) 554, 56-63 (2004).

32. Kasteleijn-Nolst Trenite, D. G. \& Hirsch, E. Levetiracetam: preliminary efficacy in generalized seizures. Epileptic Disord. 5, S39-S44 (2003).

33. Malik, A. S. et al. A novel dehydroepiandrosterone analog improves functional recovery in a rat traumatic brain injury model. J. Neurotrauma 20, 463-476 (2003).

34. Kostrubsky, V. E. et al. Evaluation of hepatotoxic potential of drugs by inhibition of bile acid transport in cultured primary human hepatocytes and intact rats. Toxicol. Sci. 76, 220-228 (2003)

35. Lindon, J. C. et al. Contemporary issues in toxicology: the role of metabonomics in toxicology and its evaluation by the COMET project. Toxicol. Appl. Pharmacol. 187, 137-146 (2003).

36. Tam, R. C. et al. The ribavirin analog ICN 17261 demonstrates reduced toxicity and antiviral effects with retention of both immunomodulatory activity and reduction of hepatitis-induced serum alanine aminotransferase levels. Antimicrob. Agents Chemother. 44, 1276-1283 (2000).

37. Youssef, A. F., Turck, P. \& Fort, F. L. Safety and pharmacokinetics of oral lansoprazole in preadolescent rats exposed from weaning through sexual maturity. Reprod. Toxicol. 17, 109-116 (2003)

38. National Institutes of Health. Network for Large-Scale Sequencing of the Rat Genome 〈http:// grants2.nih.gov/grants/guide/rfa-files/RFA-HG-00-002.html> (2000).

39. Aparicio, S. et al. Whole-genome shotgun assembly and analysis of the genome of Fugu rubripes. Science 297, 1301-1310 (2002)

40. Yu, J. et al. A draft sequence of the rice genome (Oryza sativa L. ssp. indica). Science 296, 79-92 (2002)

41. Goff, S. A. et al. A draft sequence of the rice genome (Oryza sativa L. ssp. japonica). Science 296, 92-100 (2002).

42. Adams, M. D. et al. The genome sequence of Drosophila melanogaster. Science 287, 2185-2195 (2000)

43. Dehal, P. et al. The draft genome of Ciona intestinalis: insights into chordate and vertebrate origins. Science 298, 2157-2167 (2002).

44. Myers, E. W. et al. A whole-genome assembly of Drosophila. Science 287, 2196-2204 (2000).

45. Myers, E. W., Sutton, G. G., Smith, H. O., Adams, M. D. \& Venter, J. C. On the sequencing and assembly of the human genome. Proc. Natl Acad. Sci. USA 99, 4145-4146 (2002).

46. Waterston, R. H., Lander, E. S. \& Sulston, J. E. On the sequencing of the human genome. Proc. Nat Acad. Sci. USA 99, 3712-3716 (2002)

47. Waterston, R. H., Lander, E. S. \& Sulston, J. E. More on the sequencing of the human genome. Proc Natl Acad. Sci. USA 100, 3022-3024 (2003); author reply (100), 3025-3026 (2003).

48. Green, P. Whole-genome disassembly. Proc. Natl Acad. Sci. USA 99, 4143-4144 (2002).

49. Batzoglou, S. et al. ARACHNE: a whole-genome shotgun assembler. Genome Res. 12, 177-189 (2002) 
50. Jaffe, D. B. et al. Whole-genome sequence assembly for mammalian genomes: Arachne 2. Genome Res. 13, 91-96 (2003)

51. Cheung, J. et al. Recent segmental and gene duplications in the mouse genome. Genome Biol. 4, R47 [online] (2003).

52. Eichler, E. E. Masquerading repeats: Paralogous pitfalls of the human genome. Genome Res. 8 , 758-762 (1998)

53. Eichler, E. E. Segmental duplications: what's missing, misassigned, and misassembled-and should we care? Genome Res. 11, 653-656 (2001).

54. Havlak, P. et al. The Atlas genome assembly system. Genome Res. 14, 721-732 (2004).

55. Osoegawa, K. et al. BAC Resources for the rat genome project. Genome Res. 14, 780-785 (2004).

56. Krzywinski, M. et al. Integrated and sequence-ordered BAC and YAC-based physical maps for the rat genome. Genome Res. 14, 766-779 (2004).

57. Chen, R., Sodergren, E., Gibbs, R. \& Weinstock, G. M. Dynamic building of a BAC clone tiling path for genome sequencing project. Genome Res. 14, 679-684 (2004).

58. Cai, L. et al. Construction and characterization of a 10-genome equivalent yeast artificial chromosome library for the laboratory rat, Rattus norvegicus. Genomics 39, 385-392 (1997).

59. Kwitek, A. E. et al. High density rat radiation hybrid maps containing over 24,000 SSLPs, genes, and ESTs provide a direct link to the rat genome sequence. Genome Res. 14, 750-757 (2004).

60. Felsenfeld, A., Peterson, J., Schloss, J. \& Guyer, M. Assessing the quality of the DNA sequence from the Human Genome Project. Genome Res. 9, 1-4 (1999).

61. Steen, R. G. et al. A high-density integrated genetic linkage and radiation hybrid map of the laboratory rat. Genome Res. 9 (insert), AP1-AP8 (1999).

62. Misra, S. et al. Annotation of the Drosophila melanogaster euchromatic genome: a systematic review. Genome Biol. 3, RESEARCH0083.1-0083.22 [online] (2002).

63. Li, X. \& Waterman, M. S. Estimating the repeat structure and length of DNA sequences using L-tuples. Genome Res. 13, 1916-1922 (2003).

64. Riethman, H. et al. Mapping and initial analysis of human subtelomeric sequence assemblies. Genome Res. 14, 18-28 (2004).

65. Bayona-Bafaluy, M. P. et al. Revisiting the mouse mitochondrial DNA sequence. Nucleic Acids Res. 31, 5349-5355 (2003)

66. Kent, W. J., Baertsch, R., Hinrichs, A., Miller, W. \& Haussler, D. Evolution's cauldron: duplication, deletion, and rearrangement in the mouse and human genomes. Proc. Natl Acad. Sci. USA 100, 11484-11489 (2003).

67. Pevzner, P. \& Tesler, G. Human and mouse genomic sequences reveal extensive breakpoint reuse in mammalian evolution. Proc. Natl Acad. Sci. USA 100, 7672-7677 (2003).

68. Nadeau, J. H. \& Taylor, B. A. Lengths of chromosomal segments conserved since divergence of man and mouse. Proc. Natl Acad. Sci. USA 81, 814-818 (1984).

69. Schwartz, S. et al. Human-mouse alignments with BLASTZ. Genome Res. 13, 103-107 (2003).

70. Ma, B., Tromp, J. \& Li, M. PatternHunter: faster and more sensitive homology search Bioinformatics 18, 440-445 (2002).

71. Pevzner, P. \& Tesler, G. Genome rearrangements in mammalian evolution: lessons from human and mouse genomes. Genome Res. 13, 37-45 (2003).

72. Kalafus, K. J., Jackson, A. R. \& Milosavljevic, A. Pash: Efficient genome-scale sequence anchoring by positional hashing. Genome Res. 14, 672-678 (2004).

73. Bourque, G., Pevzner, P. A. \& Tesler, G. Reconstructiong the genomic architecture of ancestral mammals: lessons from human, mouse, and rat genomes. Genome Res. 14, 507-516 (2004).

74. Graves, J. A., Gecz, J. \& Hameister, H. Evolution of the human X-a smart and sexy chromosome that controls speciation and development. Cytogenet. Genome Res. 99, 141-145 (2002).

75. Bourque, G. \& Pevzner, P. A. Genome-scale evolution: reconstructing gene orders in the ancestral species. Genome Res. 12, 26-36 (2002).

76. Murphy, W. J., Bourque, G., Tesler, G., Pevzner, P. \& O’Brien, S. J. Reconstructing the genomic architecture of mammalian ancestors using multispecies comparative maps. Hum. Genom. 1, 30-40 (2003).

77. Kirkness, E. F. et al. The dog genome: survey sequencing and comparative analysis. Science 301, 1898-1903 (2003)

78. Murphy, W. J., Sun, S., Chen, Z. Q., Pecon-Slattery, J. \& O'Brien, S. J. Extensive conservation of sex chromosome organization between cat and human revealed by parallel radiation hybrid mapping. Genome Res. 9, 1223-1230 (1999).

79. Ventura, M., Archidiacono, N. \& Rocchi, M. Centromere emergence in evolution. Genome Res. 11, 595-599 (2001).

80. Yunis, J. J. \& Prakash, O. The origin of man: a chromosomal pictorial legacy. Science 215, 1525-1530 (1982).

81. Murphy, W. J., Fronicke, L., O'Brien, S. J. \& Stanyon, R. The origin of human chromosome 1 and its homologs in placental mammals. Genome Res. 13, 1880-1888 (2003)

82. Stanyon, R., Stone, G., Garcia, M. \& Froenicke, L. Reciprocal chromosome painting shows that squirrels, unlike murid rodents, have a highly conserved genome organization. Genomics $\mathbf{8 2}$ 245-249 (2003).

83. Bailey, J. A. et al. Recent segmental duplications in the human genome. Science 297, 1003-1007 (2002).

84. Thomas, J. W. et al. Pericentromeric duplications in the laboratory mouse. Genome Res. 13, 55-63 (2003).

85. Horvath, J. E. et al. Using a pericentromeric interspersed repeat to recapitulate the phylogeny and expansion of human centromeric segmental duplications. Mol. Biol. Evol. 20, 1463-1479 (2003).

86. Guy, J. et al. Genomic sequence and transcriptional profile of the boundary between pericentromeric satellites and genes on human chromosome arm 10p. Genome Res. 13, 159-172 (2003).

87. Tuzun, E., Bailey, J. A. \& Eichler, E. E. Recent segmental duplications in the working draft assembly of the brown Norway rat. Genome Res. 14, 493-506 (2004).

88. Pruitt, K. D. \& Maglott, D. R. RefSeq and LocusLink: NCBI gene-centered resources. Nucleic Acids Res. 29, 137-140 (2001)

89. Yang, S. et al. Patterns of insertions and their covariation with substitutions in the rat, mouse and human genomes. Genome Res. 14, 517-527 (2004).

90. Roskin, K. M., Diekhans, M. \& Haussler, D. in Proc. 7th Annu. Int. Conf. Res. Comput. Mol. Biol. (RECOMB 2003) (eds Vingron, M., Istrail, S., Pevzner, P. \& Waterman, M.) doi:10.1145/ 640075.640109, 257-266 (ACM Press, New York, 2003).
91. Chiaromonte, F. et al. The share of human genomic DNA under selection estimated from humanmouse genomic alignments. Cold Spring Harbor Symp. Quant. Biol. (in the press)

92. Cooper, G. M. et al. Characterization of evolutionary rates and constraints in three mammalian genomes. Genome Res. 14, 539-548 (2004).

93. Dermitzakis, E. T. et al. Numerous potentially functional but non-genic conserved sequences on human chromosome 21. Nature 420, 578-582 (2002).

94. Dermitzakis, E. T. et al. Evolutionary discrimination of mammalian conserved non-genic sequences (CNGs). Science 302, 1033-1035 (2003).

95. Nekrutenko, A. Rat-mouse comparisons to identify rodent-specific exons. Genome Res. (in the press).

96. Hardison, R. C. et al. Covariation in frequencies of substitution, deletion, transposition, and recombination during eutherian evolution. Genome Res. 13, 13-26 (2003).

97. Thomas, J. W. et al. Comparative analyses of multi-species sequences from targeted genomic regions. Nature 424, 788-793 (2003).

98. Cooper, G. M., Brudno, M., Green, E. D., Batzoglou, S. \& Sidow, A. Quantitative estimates of sequence divergence for comparative analyses of mammalian genomes. Genome Res. 13, 813-820 (2003)

99. Huckins, C. The spermatogonial stem cell population in adult rats. I. Their morphology, proliferation and maturation. Anat. Rec. 169, 533-557 (1971).

100. Clermont, Y. Kinetics of spermatogenesis in mammals: seminiferous epithelium cycle and spermatogonial renewal. Physiol. Rev. 52, 198-236 (1972).

101. Makova, K. D., Yang, S. \& Chiaromonte, F. Insertions and deletions are male biased too: A wholegenome analysis in rodents. Genome Res. 14, 567-573 (2004).

102. Sundstrom, H., Webster, M. T. \& Ellegren, H. Is the rate of insertion and deletion mutation male biased? Molecular evolutionary analysis of avian and primate sex chromosome sequences. Genetics 164, 259-268 (2003).

103. Chang, B. H. \& Li, W. H. Estimating the intensity of male-driven evolution in rodents by using X-linked and Y-linked Ube 1 genes and pseudogenes. J. Mol. Evol. 40, 70-77 (1995).

104. Chang, B. H., Shimmin, L. C., Shyue, S. K., Hewett-Emmett, D. \& Li, W. H. Weak male-driven molecular evolution in rodents. Proc. Natl Acad. Sci. USA 91, 827-831 (1994).

105. Bird, A. P. DNA methylation and the frequency of CpG in animal DNA. Nucleic Acids Res. 8 , 1499-1504 (1980).

106. Antequera, F. \& Bird, A. Number of CpG islands and genes in human and mouse. Proc. Natl Acad. Sci. USA 90, 11995-11999 (1993).

107. Jensen-Seaman, M. I. et al. Comparative recombination rates in the rat, mouse, and human genomes. Genome Res. 14, 528-538 (2004).

108. Birdsell, J. A. Integrating genomics, bioinformatics, and classical genetics to study the effects of recombination on genome evolution. Mol. Biol. Evol. 19, 1181-1197 (2002).

109. Montoya-Burgos, J. I., Boursot, P. \& Galtier, N. Recombination explains isochores in mammalian genomes. Trends Genet. 19, 128-130 (2003).

110. Bray, N. \& Pachter, L. MAVID Constrained ancestral alignment of multiple sequence. Genome Res. 14, 693-699 (2004)

111. Yap, V. B. \& Pachter, L. Identification of evolutionary hotspots in the rodent genomes. Genome Res. 14, 574-579 (2004)

112. Hubbard, T. et al. The Ensembl genome database project. Nucleic Acids Res. 30, 38-41 (2002)

113. Vitt, U. et al. Identification of candidate disease genes by EST alignments, synteny and expression and verification of Ensembl genes on rat chromosome 1q43-54. Genome Res. 14, 640-650 (2004).

114. Burge, C. \& Karlin, S. Prediction of complete gene structures in human genomic DNA. J. Mol. Biol. 268, 78-94 (1997).

115. Guigo, R., Knudsen, S., Drake, N. \& Smith, T. Prediction of gene structure. J. Mol. Biol. 226, 141-157 (1992).

116. Solovyev, V. V., Salamov, A. A. \& Lawrence, C. B. Identification of human gene structure using linear discriminant functions and dynamic programming. Proc. Int. Conf. Intell. Syst. Mol. Biol. 3 367-375 (1995).

117. Parra, G. et al. Comparative gene prediction in human and mouse. Genome Res. 13, 108-117 (2003).

118. Alexandersson, M., Cawley, S. \& Pachter, L. SLAM—Cross-species gene finding with a generalized pair hidden Markov model. Genome Res. 13, 496-502 (2003).

119. Flicek, P., Keibler, E., Hu, P., Korf, I. \& Brent, M. R. Leveraging the mouse genome for gene prediction in human: from whole-genome shotgun reads to a global synteny map. Genome Res. 13, 46-54 (2003).

120. Korf, I., Flicek, P., Duan, D. \& Brent, M. R. Integrating genomic homology into gene structure prediction. Bioinformatics 17 (suppl. 1), S140-S148 (2001)

121. Wu, J. Q., Shteynberg, D., Arumugam, M., Gibbs, R. A. \& Brent, M. R. Identification of rat genes by TWINSCAN gene prediction, RT-PCR, and direct sequencing. Genome Res. 14, 655-671 (2004).

122. Dewey, C. et al. Accurate identification of novel human genes through simultaneous gene prediction in human, mouse and rat. Genome Res. 14, 661-664 (2004).

123. Kent, W. J. BLAT-the BLAST-like alignment tool. Genome Res. 12, 656-664 (2002).

124. Puente, X. S. \& Lopez-Otin, C. A. A genomic analysis of rat proteases and protease inhibitors. Genome Res. 14, 609-622 (2004)

125. Puente, X. S., Sanchez, L. M., Overall, C. M. \& Lopez-Otin, C. Human and mouse proteases: comparative genomic approach. Nature Rev. Genet. 4, 544-558 (2003).

126. Hurst, L. D. The Ka/Ks ratio: diagnosing the form of sequence evolution. Trends Genet. 18, 486-487 (2002)

127. Makalowski, W. \& Boguski, M. S. Evolutionary parameters of the transcribed mammalian genome: an analysis of 2,820 orthologous rodent and human sequences. Proc. Natl Acad. Sci. USA 95 9407-9412 (1998).

128. Wolfe, K. H. \& Sharp, P. M. Mammalian gene evolution: nucleotide sequence divergence between mouse and rat. J. Mol. Evol. 37, 441-456 (1993).

129. Modrek, B. \& Lee, C. J. Alternative splicing in the human, mouse and rat genomes is associated with an increased frequency of exon creation and/or loss. Nature Genet. 34, 177-180 (2003).

130. Nekrutenko, A., Makova, K. D. \& Li, W. H. The $\mathrm{K}_{\mathrm{A}} / \mathrm{K}_{\mathrm{S}}$ ratio test for assessing the proteincoding potential of genomic regions: an empirical and simulation study. Genome Res. 12, 198-202 (2002). 
131. Nekrutenko, A., Chung, W. Y. \& Li, W. H. An evolutionary approach reveals a high protein-coding capacity of the human genome. Trends Genet. 19, 306-310 (2003).

132. Taylor, M. S., Ponting, C. P. \& Copley, R. R. Occurrence and consequences of coding sequence insertions and deletions in mammalian genomes. Genome Res. 14, 555-566 (2004).

133. Green, H. \& Wang, N. Codon reiteration and the evolution of proteins. Proc. Natl Acad. Sci. USA 91 , 4298-4302 (1994).

134. Levinson, G. \& Gutman, G. A. Slipped-strand mispairing: a major mechanism for DNA sequence evolution. Mol. Biol. Evol. 4, 203-221 (1987).

135. Alba, M. M. \& Guigo, R. Comparative analysis of amino acid repeats in rodents and humans. Genome Res. 14, 549-554 (2004).

136. Alba, M. M., Santibanez-Koref, M. F. \& Hancock, J. M. Conservation of polyglutamine tract size between mice and humans depends on codon interruption. Mol. Biol. Evol. 16, 1641-1644 (1999).

137. Burge, C. B., Padgett, R. A. \& Sharp, P. A. Evolutionary fates and origins of U12-type introns. Mol. Cell 2, 773-785 (1998).

138. Ohno, S. Evolution by Gene Duplication (Springer, Berlin, 1970).

139. Emes, R. D., Goodstadt, L., Winter, E. E. \& Ponting, C. P. Comparison of the genomes of human and mouse lays the foundation of genome zoology. Hum. Mol. Genet. 12, 701-709 (2003).

140. Lynch, M. \& Conery, J. S. The evolutionary fate and consequences of duplicate genes. Science 290, 1151-1155 (2000).

141. Prince, V. E. \& Pickett, F. B. Splitting pairs: the diverging fates of duplicated genes. Nature Rev Genet. 3, 827-837 (2002).

142. Hughes, A. L. Adaptive Evolution of Genes and Genomes Ch. 7, 143-179 (Oxford Univ. Press, New York, 1999).

143. Tagle, D. A. et al. Embryonic epsilon and $\gamma$ globin genes of a prosimian primate (Galago crassicaudatus). Nucleotide and amino acid sequences, developmental regulation and phylogenetic footprints. J. Mol. Biol. 203, 439-455 (1988).

144. Altschul, S. F. \& Lipman, D. J. Protein database searches for multiple alignments. Proc. Natl Acad. Sci. USA 87, 5509-5513 (1990).

145. Gumucio, D. L. et al. Phylogenetic footprinting reveals a nuclear protein which binds to silencer sequences in the human $\gamma$ and $\varepsilon$ globin genes. Mol. Cell. Biol. 12, 4919-4929 (1992).

146. Hardison, R. et al. Comparative analysis of the locus control region of the rabbit $\beta$-like gene cluster: HS3 increases transient expression of an embryonic $\varepsilon$-globin gene. Nucleic Acids Res. 21, 1265-1272 (1993).

147. Boffelli, D. et al. Phylogenetic shadowing of primate sequences to find functional regions of the human genome. Science 299, 1391-1394 (2003).

148. Elnitski, L. et al. Distinguishing regulatory DNA from neutral sites. Genome Res. 13, 64-72 (2003).

149. Loots, G. G., Ovcharenko, I., Pachter, L., Dubchak, I. \& Rubin, E. M. rVista for comparative sequence-based discovery of functional transcription factor binding sites. Genome Res. 12, 832-839 (2002).

150. Pennacchio, L. A. \& Rubin, E. M. Genomic strategies to identify mammalian regulatory sequences. Nature Rev. Genet. 2, 100-109 (2001).

151. Margulies, E. H., Blanchette, M., Haussler, D. \& Green, E. Identification and characterization of multi-species conserved sequences. Genome Res. 13, 2507-2518 (2003).

152. Kolbe, D., et al. Regulatory potential scores from genome-wide 3-way alignments of human, mouse and rat. Genome Res. 14, 700-707 (2004).

153. Wingender, E. et al. The TRANSFAC system on gene expression regulation. Nucleic Acids Res. 29, 281-283 (2001).

154. Trinklein, N. D., Aldred, S. J., Saldanha, A. J. \& Myers, R. M. Identification and functional analysis of human transcriptional promoters. Genome Res. 13, 308-312 (2003).

155. Philipsen, S., Pruzina, S. \& Grosveld, F. The minimal requirements for activity in transgenic mice of hypersensitive site 3 of the $\beta$ globin locus control region. EMBO J. 12, 1077-1085 (1993).

156. Reddy, P. M. \& Shen, C. K. Protein-DNA interactions in vivo of an erythroid-specific, human $\beta$-globin locus enhancer. Proc. Natl Acad. Sci. USA 88, 8676-8680 (1991).

157. Strauss, E. C. \& Orkin, S. H. In vivo protein-DNA interactions at hypersensitive site 3 of the human $\beta$-globin locus control region. Proc. Natl Acad. Sci. USA 89, 5809-5813 (1992).

158. Hillier, L. W. et al. The DNA sequence of human chromosome 7. Nature 424, 157-164 (2003).

159. Torrents, D., Suyama, M. \& Bork, P. A genome-wide survey of human pseudogenes. Genome Res. 13, 2559-2567 (2003).

160. Zhang, Z., Harrison, P. \& Gerstein, M. Identification and analysis of over 2000 ribosomal protein pseudogenes in the human genome. Genome Res. 12, 1466-1482 (2002).

161. Mulder, N. J. et al. The InterPro Database 2003 brings increased coverage and new features. Nucleic Acids Res. 31, 315-318 (2003)

162. Oh, B., Hwang, S. Y., Solter, D. \& Knowles, B. B. Spindlin, a major maternal transcript expressed in the mouse during the transition from oocyte to embryo. Development 124, 493-503 (1997)

163. Garcia-Meunier, P., Etienne-Julan, M., Fort, P., Piechaczyk, M. \& Bonhomme, F. Concerted evolution in the GAPDH family of retrotransposed pseudogenes. Mamm. Genome 4, 695-703 (1993).

164. Smit, A. F. Interspersed repeats and other mementos of transposable elements in mammalian genomes. Curr. Opin. Genet. Dev. 9, 657-663 (1999).

165. Prak, E. T. \& Kazazian, H. H. Jr Mobile elements and the human genome. Nature Rev. Genet. 1, 134-144 (2000).

166. Ostertag, E. M. \& Kazazian, H. H. Jr Biology of mammalian L1 retrotransposons. Annu. Rev. Genet. 35, 501-538 (2001).

167. Weiner, A. M. SINEs and LINEs: the art of biting the hand that feeds you. Curr. Opin. Cell Biol. 14, $343-350$ (2002).

168. Martin, S. L. \& Bushman, F. D. Nucleic acid chaperone activity of the ORF1 protein from the mouse LINE-1 retrotransposon. Mol. Cell. Biol. 21, 467-475 (2001).

169. Hayward, B. E., Zavanelli, M. \& Furano, A. V. Recombination creates novel L1 (LINE-1) elements in Rattus norvegicus. Genetics 146, 641-654 (1997)

170. Dewannieux, M., Esnault, C. \& Heidmann, T. LINE-mediated retrotransposition of marked Alu sequences. Nature Genet. 35, 41-48 (2003).

171. Quentin, Y. A master sequence related to a free left Alu monomer (FLAM) at the origin of the B family in rodent genomes. Nucleic Acids Res. 22, 2222-2227 (1994).
172. Cantrell, M. A. et al. An ancient retrovirus-like element contains hot spots for SINE insertion. Genetics 158, 769-777 (2001)

173. Rothenburg, S., Eiben, M., Koch-Nolte, F. \& Haag, F. Independent integration of rodent identifier (ID) elements into orthologous sites of some RT6 alleles of Rattus norvegicus and Rattus rattus. J. Mol. Evol. 55, 251-259 (2002).

174. Roy-Engel, A. M. et al. Non-traditional Alu evolution and primate genomic diversity. J. Mol. Biol. 316, 1033-1040 (2002).

175. Salem, A. H., Kilroy, G. E., Watkins, W. S., Jorde, L. B. \& Batzer, M. A. Recently integrated Alu elements and human genomic diversity. Mol. Biol. Evol. 20, 1349-1361 (2003).

176. Salem, A. H. et al. Alu elements and hominid phylogenetics. Proc. Natl Acad. Sci. USA 100, 12787-127891 (2003).

177. Smit, A. F. Identification of a new, abundant superfamily of mammalian LTR-transposons. Nucleic Acids Res. 21, 1863-1872 (1993).

178. Benit, L. et al. Cloning of a new murine endogenous retrovirus, MuERV-L, with strong similarity to the human HERV-L element and with a gag coding sequence closely related to the Fvl restriction gene. J. Virol. 71, 5652-5657 (1997).

179. Costas, J. Molecular characterization of the recent intragenomic spread of the murine endogenous retrovirus MuERV-L. J. Mol. Evol. 56, 181-186 (2003).

180. Emes, R. D., Beatson, S. A., Ponting, C. P. \& Goodstadt, L. Evolution and comparative genomics of odorant- and pheromone-associated genes in rodents. Genome Res. 14, 591-602 (2004).

181. Young, J. M. et al. Different evolutionary processes shaped the mouse and human olfactory receptor gene families. Hum. Mol. Genet. 11, 535-546 (2002).

182. Zhang, X. \& Firestein, S. The olfactory receptor gene superfamily of the mouse. Nature Neurosci. 5, 124-133 (2002).

183. Rouquier, S., Blancher, A. \& Giorgi, D. The olfactory receptor gene repertoire in primates and mouse: evidence for reduction of the functional fraction in primates. Proc. Natl Acad. Sci. USA 97, 2870-2874 (2000).

184. Clark, A. J., Hickman, J. \& Bishop, J. A 45-kb DNA domain with two divergently orientated genes is the unit of organisation of the murine major urinary protein genes. EMBO J. 3, 2055-2064 (1984).

185. Mural, R. J. et al. A comparison of whole-genome shotgun-derived mouse chromosome 16 and the human genome. Science 296, 1661-1671 (2002).

186. Cavaggioni, A. \& Mucignat-Caretta, C. Major urinary proteins, $\alpha_{2 \mathrm{U}}$-globulins and aphrodisin. Biochim. Biophys. Acta 1482, 218-228 (2000).

187. Hurst, J. L. et al. Individual recognition in mice mediated by major urinary proteins. Nature $\mathbf{4 1 4}$, 631-634 (2001).

188. Danielson, P. B. The cytochrome P450 superfamily: biochemistry, evolution and drug metabolism in humans. Curr. Drug Metab. 3, 561-597 (2002).

189. Nelson, D. R. Cytochrome P450 and the individuality of species. Arch. Biochem. Biophys. 369, 1-10 (1999).

190. Scarborough, P. E., Ma, J., Qu, W. \& Zeldin, D. C. P450 subfamily CYP2J and their role in the bioactivation of arachidonic acid in extrahepatic tissues. Drug Metab. Rev. 31, 205-234 (1999).

191. Willson, T. M. \& Kliewer, S. A. PXR, CAR and drug metabolism. Nature Rev. Drug Discov. 1, 259-266 (2002).

192. Gurates, B. et al. WT1 and DAX-1 inhibit aromatase P450 expression in human endometrial and endometriotic stromal cells. J. Clin. Endocrinol. Metab. 87, 4369-4377 (2002).

193. Zhang, Z. et al. Genomic analysis of the nuclear receptor family: new insights into structure, regulation, and evolution from the rat genome. Genome Res. 14, 580-590 (2004).

194. Lopez-Otin, C. \& Overall, C. M. Protease degradomics: a new challenge for proteomics. Nature Rev. Mol. Cell Biol. 3, 509-519 (2002).

195. Stenson, P. D. et al. Human Gene Mutation Database (HGMD): 2003 update. Hum. Mutat. 21, 577-581 (2003).

196. Huang, H. et al. Evolutionary conservation of human disease gene orthologs in the rat and mouse genomes. Genome Biol. (submitted).

197. Duret, L. \& Mouchiroud, D. Determinants of substitution rates in mammalian genes: expression pattern affects selection intensity but not mutation rate. Mol. Biol. Evol. 17, 68-74 (2000)

198. Reddy, P. S. \& Housman, D. E. The complex pathology of trinucleotide repeats. Curr. Opin. Cell Biol. 9, 364-372 (1997).

199. The International HapMap Consortium. The International HapMap Project. Nature 426, 789-796 (2003)

200. Wade, C. M. et al. The mosaic structure of variation in the laboratory mouse genome. Nature $\mathbf{4 2 0}$, 574-578 (2002).

201. Zimdahl, H. et al. A SNP map of the rat genome generated from cDNA sequences. Science 303, 807 (2004).

202. Mendell, J. T. \& Dietz, H. C. When the message goes awry: disease-producing mutations that influence mRNA content and performance. Cell 107, 411-414 (2001).

203. Venter, J. C. et al. The sequence of the human genome. Science 291, 1304-1351 (2001)

204. Marra, M. et al. A map for sequence analysis of the Arabidopsis thaliana genome. Nature Genet. 22, 265-270 (1999).

205. Marra, M. A. et al. High throughput fingerprint analysis of large-insert clones. Genome Res. 7, 1072-1084 (1997).

206. The International Human Genome Mapping Consortium. A physical map of the human genome. Nature 409, 934-941 (2001).

207. Schein, J. E. A. in Bacterial Artificial Chromosomes: Methods and Protocols (eds Zhao, S. \& Stodolsky, M.) 143-156 (Humana, Totowa, New Jersey, 2004).

208. Soderlund, C. I. et al. FPC: a system for building contigs from restriction fingerprinted clones. Comput. Appl. Biosci. 13, 523-535 (1997).

209. Soderlund, C. S. et al. Contigs built with fingerprints, markers, and FPC V4.7. Genome Res. 10, 1772-1787 (2000).

210. Ness, S. R. et al. Assembly of fingerprint contigs: parallelized FPC. Bioinformatics 18, 484-485 (2002).

211. Woon, P. Y. et al. Construction and characterization of a 10-fold genome equivalent rat P1-derived artificial chromosome library. Genomics 50, 306-316 (1998).

212. Watanabe, T. K. et al. A radiation hybrid map of the rat genome containing 5,255 markers. Nature Genet. 22, 27-36 (1999). 
213. Schuler, G. D. Sequence mapping by electronic PCR Genome Res. 7, 541-550 (1997).

214. Benson, G. Tandem repeats finder: a program to analyze DNA sequences. Nucleic Acids Res. 27, 573-580 (1999).

215. Blanchette, M. et al. Aligning multiple genomic sequences with the threaded blockset aligner. Genome Res. 14, 708-715 (2004)

216. Brudno, M. et al. LAGAN and Multi-LAGAN: efficient tools for large-scale multiple alignment of genomic DNA. Genome Res. 13, 721-731 (2003).

217. Brudno, M. et al. Automated whole-genome multiple alignment of rat, mouse, and human. Genome Res. 14, 685-692 (2004)

218. Bailey, J. A., Yavor, A. M., Massa, H. F., Trask, B. J. \& Eichler, E. E. Segmental duplications: organization and impact within the current human genome project assembly. Genome Res. 11, 1005-1017 (2001)

219. Schwartz, S. et al. MultiPipMaker and supporting tools: Alignments and analysis of multiple genomic DNA sequences. Nucleic Acids Res. 31, 3518-3524 (2003).

220. Altschul, S. F. et al. Gapped BLAST and PSI-BLAST: a new generation of protein database search programs. Nucleic Acids Res. 25, 3389-3402 (1997).

221. Birney, E. \& Durbin, R. Using GeneWise in the Drosophila annotation experiment. Genome Res. 10 547-548 (2000).

222. Yang, Z., Goldman, N. \& Friday, A. Comparison of models for nucleotide substitution used in maximum-likelihood phylogenetic estimation. Mol. Biol. Evol. 11, 316-324 (1994).

223. Chakrabarti, K. \& Pachter, L. Visualization of multiple genome annotations and alignments with the K-BROWSER. Genome Res. 14, 716-720 (2004).

224. Saitou, N. \& Nei, M. The neighbor-joining method: a new method for reconstructing phylogenetic trees. Mol. Biol. Evol. 4, 406-425 (1987).

225. Haldi, M. L. et al. Construction of a large-insert yeast artificial chromosome library of the rat genome. Mamm. Genome 8, 284 (1997).

Supplementary Information accompanies the paper on www.nature.com/nature.
Acknowledgements Work at Baylor College of Medicine was supported by a grant from the NHGRI and NHLBI to R.A.G. Work at Genome Therapeutics was supported by grants from the NHGRI to D.S. A.S. acknowledges support from the NIGMS. M.B. acknowledges support from the NIH. N.H. was supported by the NGFN/BMBF (German Ministry for Research and Education). B.J.T. and J.M.Y. are supported by an NIH grant from the NIDCD. K.M.R. and G.M.C. are Howard Hughes Medical Institute Predoctoral Fellows. L.M.D'S., K.M. and K.J.K. are supported by training fellowships from the W. M. Keck Foundation to the Gulf Coast Consortia through the Keck Center for Computational and Structural Biology. Work at Case Western Reserve was supported in part by NIH grants to E.E.E. Work at IMIM was supported by a grant from Plan Nacional de I + D (Spain). M.M.A. acknowledges support from programme Ramón y Cajal and a grant from the Spanish Ministry of Science and Technology. Work at Universidad de Oviedo was supported by grants from the European Union, Obra Social Cajastur and Gobierno del Principado de Asturias. Work at Penn State University was supported by NHGRI grants. Work at the University of California Berkeley was supported by a grant from the NIH. Work at the Washington University School of Medicine Genome Sequencing Center and the British Columbia Cancer Agency Genome Sciences Centre was supported by an NIH grant. Work at UCSC and CHORI was supported by the NHGRI.

Competing interests statement The authors declare that they have no competing financial interests.

Correspondence and requests for materials should be addressed to R.A.G. (agibbs@bcm.tmc.edu). The genomic sequence is available under accession numbers AABR03000000 to AABR03137910 in the international sequence databases (GenBank, DDBJ and EMBL)

Rat Genome Sequencing Project Consortium (Participants are arranged under area of contribution, and then by institution.)

DNA sequencing: Baylor College of Medicine Richard A. Gibbs (Principal Investigator)', George M. Weinstock (Co-principal Investigator)'1, Michael L. Metzker', Donna M. Muzny', Erica J. Sodergren', Steven Scherer', Graham Scott', David Steffen', Kim C. Worley', Paula E. Burch', Geoffrey Okwuonu', Sandra Hines', Lora Lewis', Christine DeRamo', Oliver Delgado', Shannon Dugan-Rocha', George Miner', Margaret Morgan', Alicia Hawes', Rachel Gill'; Celera Robert A. Holt (Principal Investigator) ${ }^{2,3}$, Mark D. Adams ${ }^{3,4}$, Peter G. Amanatides ${ }^{3,5}$, Holly Baden-Tillson ${ }^{3,6}$, Mary Barnstead $^{3,7}$, Soo Chin ${ }^{3}$, Cheryl A. Evans ${ }^{3}$, Steve Ferriera, ${ }^{3,8}$, Carl Fosler ${ }^{3}$, Anna Glodek, ${\text {, Zhiping Gu }{ }^{3} \text {, Don Jennings }{ }^{3} \text {, Cheryl L. Kraft }}^{3,10}$, Trixie Nguyen $^{3}$, Cynthia M. Pfannkoch ${ }^{3,6}$, Cynthia Sitter ${ }^{3,11}$, Granger G. Sutton ${ }^{3}$, J. Craig Venter ${ }^{3,8}$, Trevor Woodage ${ }^{3}$; Genome Therapeutics Douglas Smith (Principal Investigator) $^{12,13}$, Hong-Mei Lee ${ }^{12}$, Erik Gustafson ${ }^{12,13}$, Patrick Cahill ${ }^{12}$, Arnold Kana ${ }^{12}$, Lynn Doucette-Stamm ${ }^{12,13}$, Keith Weinstock $^{12}$, Kim Fechtel ${ }^{12}$; University of Utah Robert B. Weiss (Principal Investigator) ${ }^{14}$, Diane M. Dunn ${ }^{14}$; NISC Comparative Sequencing Program, NHGRI Eric D. Green ${ }^{15}$, Robert W. Blakesley ${ }^{15}$, Gerard G. Bouffard ${ }^{15}$

BAC library production: Children's Hospital Oakland Research Institute Pieter J. de Jong (Principal Investigator) ${ }^{16}$, Kazutoyo Osoegawa ${ }^{16}$, Baoli Zhu' ${ }^{16}$

BAC fingerprinting: British Columbia Cancer Agency, Canada's Michael Smith Genome Sciences Centre Marco Marra (Principal Investigator)², Jacqueline Schein (Principal Investigator) ${ }^{2}$, lan Bosdet², Chris Fjell2, Steven Jones², Martin Krzywinski², Carrie Mathewson², Asim Siddiqui², Natasja Wye'; Genome Sequencing Center, Washington University School of Medicine John McPherson ${ }^{1,17}$

BAC end sequencing: TIGR Shaying Zhao (Principal Investigator) ${ }^{18}$, Claire M. Fraser ${ }^{18}$, Jyoti Shetty ${ }^{18}$, Sofiya Shatsman ${ }^{18}$, Keita Geer ${ }^{18}$, Yixin Chen ${ }^{18}$, Sofyia Abramzon ${ }^{18}$, William C. Nierman ${ }^{18}$

Sequence assembly: Baylor College of Medicine Richard A. Gibbs (Principal Investigator) ${ }^{1}$, George M. Weinstock (Principal Investigator)', Paul H. Havlak', Rui Chen', K. James Durbin', Amy Egan', Yanru Ren', Xing-Zhi Song', Bingshan Li', Yue Liu', Xiang Qin'

Analysis and annotation: Affymetrix Simon Cawley ${ }^{19}$; Baylor College of Medicine George M. Weinstock (Coordinator) ${ }^{1}$, Kim C. Worley (Overall Coordinator) ${ }^{1}$, A. J. Cooney ${ }^{20}$, Richard A. Gibbs', Lisa M. D'Souza', Kirt Martin', Jia Qian Wu', Manuel L. Gonzalez-Garay', Andrew R. Jackson', Kenneth J. Kalafus ${ }^{1,58}$, Michael P. McLeod', Aleksandar Milosavljevic ${ }^{1}$, Davinder Virk', Andrei Volkov', David A. Wheeler', Zhengdong Zhang'; Case Western Reserve University Jeffrey A. Bailey ${ }^{4}$, Evan E. Eichler ${ }^{4}$, Eray Tuzun ${ }^{4}$; EBI, Wellcome Trust Genome Campus Ewan Birney $^{21}$, Emmanuel Mongin ${ }^{21}$, Abel Ureta-Vidal ${ }^{21}$, Cara Woodwark ${ }^{21}$; EMBL, Heidelberg Evgeny Zdobnov ${ }^{22}$, Peer Bork ${ }^{22,23}$, Mikita Suyama ${ }^{22}$, David Torrents ${ }^{22}$; Fraunhofer-Chalmers Research Centre for Industrial Mathematics, Gothenburg Marina Alexandersson ${ }^{24}$; Fred Hutchinson Cancer Research Center Barbara J. Trask ${ }^{25}$, Janet M. Young ${ }^{25}$; Genome Therapeutics Douglas Smith (Principal Investigator ${ }^{12,13}$, Hui Huang ${ }^{12}$, Kim Fechtel ${ }^{12}$, Huajun Wang ${ }^{12}$, Heming Xing ${ }^{12}$, Keith Weinstock ${ }^{12}$; Incyte Corporation Sue Daniels ${ }^{26}$, Darryl Gietzen ${ }^{26}$, Jeanette Schmidt ${ }^{26}$, Kristian Stevens ${ }^{26}$, Ursula Vitt ${ }^{26}$, Jim Wingrove ${ }^{26}$; Institut Municipal d'Investigacio Medica, Barcelona Francisco Camara ${ }^{27}$, M. Mar Albà' ${ }^{27}$, Josep F. Abril2 ${ }^{27}$, Roderic Guigo ${ }^{27}$; The Institute for Systems Biology Arian Smit ${ }^{28}$; Lawrence Berkeley National Laboratory Inna Dubchak $^{29,30}$, Edward M. Rubin ${ }^{29,30}$, Olivier Couronne ${ }^{29,30}$, Alexander Poliakov ${ }^{29}$; Max Delbrück Center for Molecular Medicine Norbert Hübner ${ }^{23}$, Detlev Ganten ${ }^{23}$, Claudia Goesele ${ }^{23,31}$, Oliver Hummel ${ }^{23,31}$, Thomas Kreitler ${ }^{23,31}$, Young-Ae Lee ${ }^{23}$, Jan Monti ${ }^{23}$, Herbert Schulz ${ }^{23}$, Heike Zimdahl' ${ }^{23}$; 
Max Planck Institute for Molecular Genetics, Berlin Heinz Himmelbauer ${ }^{31}$, Hans Lehrach ${ }^{31}$; Medical College of Wisconsin Howard J. Jacob (Principal Investigator) ${ }^{32}$, Susan Bromberg ${ }^{33}$, Jo Gullings-Handley ${ }^{32}$, Michael I. Jensen-Seaman ${ }^{32}$, Anne E. Kwitek ${ }^{32}$, Jozef Lazar ${ }^{32}$, Dean Pasko ${ }^{33}$, Peter J. Tonellato ${ }^{32}$, Simon Twigger ${ }^{32}$; MRC Functional Genetics Unit, University of Oxford Chris P. Ponting (Leader, Genes and Proteins Analysis

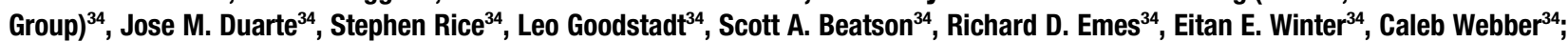
MWG-Biotech Petra Brandt ${ }^{35}$, Gerald Nyakatura ${ }^{35}$; Pennsylvania State University Margaret Adetobi ${ }^{36}$, Francesca Chiaromonte ${ }^{36}$, Laura Elnitski ${ }^{36}$, Pallavi Eswara ${ }^{36}$, Ross C. Hardison ${ }^{36}$, Minmei Hou ${ }^{36}$, Diana Kolbe ${ }^{36}$, Kateryna Makova ${ }^{36}$, Webb Miller ${ }^{36}$, Anton Nekrutenko ${ }^{36}$, Cathy Riemer ${ }^{36}$, Scott Schwartz ${ }^{36}$, James Taylor ${ }^{36}$, Shan Yang ${ }^{36}$, Yi Zhang ${ }^{36}$; Roche Genetics and Roche Center for Medical Genomics Klaus Lindpaintner ${ }^{37}$; Sanger Institute T. Dan Andrews ${ }^{38}$, Mario Caccamo ${ }^{38}$, Michele Clamp ${ }^{38}$, Laura Clarke ${ }^{38}$, Valerie Curwen ${ }^{38}$, Richard Durbin ${ }^{38}$, Eduardo Eyras ${ }^{38}$, Stephen M. Searle ${ }^{38}$; Stanford University Gregory M. Cooper (Co-Leader, Evolutionary Analysis Group) ${ }^{39}$, Serafim Batzoglou ${ }^{40}$, Michael Brudno ${ }^{40}$, Arend Sidow $^{39}$, Eric A. Stone ${ }^{39}$; The Center for the Advancement of Genomics J. Craig Venter ${ }^{3,8}$; University of Arizona Bret A. Payseur ${ }^{41}$; Université de Montréal Guillaume Bourque ${ }^{42}$; Universidad de Oviedo Carlos López-0tín ${ }^{43}$, Xose S. Puente ${ }^{43}$; University of California, Berkeley Kushal Chakrabarti ${ }^{44}$, Sourav Chatterji ${ }^{44}$, Colin Dewey ${ }^{44}$, Lior Pachter ${ }^{45}$, Nicolas Bray ${ }^{45}$, Von Bing Yap ${ }^{45}$, Anat Caspi ${ }^{46}$; University of California, San Diego Glenn Tesler $^{47}$, Pavel A. Pevzner ${ }^{48}$; University of California, Santa Cruz David Haussler (Co-Leader, Evolutionary Analysis Group) ${ }^{49}$, Krishna M. Roskin ${ }^{50}$, Robert Baertsch ${ }^{50}$, Hiram Clawson ${ }^{50}$, Terrence S. Furey ${ }^{50}$, Angie S. Hinrichs ${ }^{50}$, Donna Karolchik ${ }^{50}$, William J. Kent ${ }^{50}$, Kate R. Rosenbloom ${ }^{50}$, Heather Trumbower ${ }^{50}$, Matt Weirauch ${ }^{36,50}$; University of Wales College of Medicine David N. Cooper ${ }^{51}$, Peter D. Stenson ${ }^{51}$; University of Western Ontario Bin $\mathrm{Ma}^{52}$; Washington University Michael Brent ${ }^{53}$, Manimozhiyan Arumugam ${ }^{53}$, David Shteynberg ${ }^{53}$; Wellcome Trust Centre for Human Genetics, University of Oxford Richard R. Copley ${ }^{54}$, Martin S. Taylor ${ }^{54}$; The Wistar Institute Harold Riethman ${ }^{55}$, Uma Mudunuri55

\section{Scientific management: Jane Peterson ${ }^{56}$, Mark Guyer ${ }^{56}$, Adam Felsenfeld ${ }^{56}$, Susan Old $^{57}$, Stephen Mockrin ${ }^{57}$ \& Francis Collins ${ }^{56}$}

Affiliations for participants: 1, Human Genome Sequencing Center, Department of Molecular and Human Genetics, Baylor College of Medicine, MS BCM226, One Baylor Plaza, Houston, Texas 77030, USA 〈http://www.hgsc.bcm.tmc.edu〉; 2, British Columbia Cancer Agency, Canada's Michael Smith Genome Sciences Centre, 600 W 10th Avenue, Vancouver, British Columbia V5Z 4E6, Canada 〈http://www.bcgsc.ca); 3, Celera, 45 West Gude Drive, Rockville, Maryland 20850, USA; 4, Department of Genetics and the Center for Computational Genomics, Case Western Reserve University, School of Medicine, 10900 Euclid Avenue, Cleveland, Ohio 44106, USA; 5, DSM Pharmaceuticals Inc., 5900 NW Greenville Blvd, Greenville, North Carolina 27834, USA; 6, The Institute for Biological Energy Alternatives (IBEA), 1901 Research Blvd, Rockville, Maryland 20850, USA; 7, Intronn, Inc., 910 Clopper Road, South Building, Gaithersburg, Maryland 20878, USA; 8, The Center for the Advancement of Genomics (TCAG), 1901 Research Blvd, Suite 600, Rockville, Maryland 20850, USA; 9, Avalon Pharmaceuticals, 20358 SenecaMeadows Parkway, Germantown, Maryland 20876, USA; 10, Basic Immunology Branch, Division of Allergy, Immunology and Transplantation, National Institute of Allergy and Infectious Diseases (NIAID), NIH, DHHS, 6610 Rockledge Blvd, Room 3005, Bethesda, Maryland 20892-7612, USA; 11, DynPort Vaccine Company, LLC, 64 Thomas Jefferson Drive, Frederick, Maryland 21702, USA; 12, Genome Therapeutics Corporation, 100 Beaver Street, Waltham, Massachusetts 02453, USA; 13, Agencourt Bioscience Corporation, 100 Cummings Center, Beverly, Massachusetts 01915, USA; 14, Department of Human Genetics, University of Utah, Salt Lake City, Utah 84112, USA; 15, NIH Intramural Sequencing Center (NISC) and Genome Technology Branch, National Human Genome Research Institute (NHGRI), National Institutes of Health, Bethesda, Maryland 20892, USA; 16, BACPAC Resources, Children's Hospital Oakland Research Institute, 747 52nd Street, Oakland, California 94609, USA $\langle$ http://bacpac.chori.org〉; 17, Genome Sequencing Centre, Washington University School of Medicine, 4444 Forest Park Blvd, St Louis, Missouri 63108, USA $\langle$ http://genome.wustl.edu〉; 18, The Institute for Genomic Research, 9712 Medical Center Dr., Rockville, Maryland 20850, USA 〈http://www.tigr.org〉; 19, Affymetrix, 6550 Vallejo St, Suite 100, Emeryville, California 94608, USA; 20, Department of Cell Biology, Baylor College of Medicine, One Baylor Plaza, Houston, Texas 77030, USA; 21, EBI, Wellcome Trust Genome Campus, Hinxton, Cambridgeshire CB10 1SD, UK; 22, EMBL, Meyerhofstrasse 1, Heidelberg 69117, Germany; 23, Max Delbrück Center for Molecular Medicine (MDC), Experimental Genetics of Cardiovascular Disease, Robert-Rössle-Strasse 10, Berlin 13125, Germany $\langle$ http://www.mdc-berlin.de/ratgenome/); 24, Fraunhofer-Chalmers Research Centre for Industrial Mathematics, Chalmers Science Park, S-412 88 Gothenburg, Sweden; 25, Division of Human Biology, Fred Hutchinson Cancer Research Center, 1100 Fairview Avenue N., C3-168, Seattle, Washington 98109, USA 〈http://www.fhcrc.org/ labs/trask/); 26, Incyte Corporation, 3160 Porter Drive, Palo Alto, California 94304, USA 〈http://www.incyte.com〉; 27, Grup de Recerca en Informàtica Biomèdia, Institut Municipal d'Investigacio Medica, Universitat Pompeu Fabra, and Programa de Bioinfomatica i Genomica, Centre de Regulacio Genomica, C/ Dr. Aiguader 80, 08003 Barcelona, Catalonia, Spain; 28, Computational Biology Group, The Institute for Systems Biology, 1441 North 34th Street, Seattle, Washington 98103, USA; 29, Genomics Division, Lawrence Berkeley National Laboratory, 1 Cyclotron Rd., Berkeley, California 94720, USA 〈http://www.lbl.gov〉; 30, US Department of Energy Joint Genome Institute, 2800 Mitchell Drive, Walnut Creek, California 94598, USA 〈http://jgi.doe.gov〉; 31, Max Planck Institute for Molecular Genetics, Ihnestrasse 73, Berlin 14195, Germany; 32, Human and Molecular Genetics Center, Bioinformatics Research Center, and Department of Physiology, Medical College of Wisconsin, Milwaukee, Wisconsin 53226, USA; 33, Rat Genome Database, Bioinformatics Research Center, Medical College of Wisconsin, Milwaukee, Wisconsin 53226, USA; 34, MRC Functional Genetics Unit, University of Oxford, Department of Human Anatomy and Genetics, South Parks Road, Oxford OX1 3QX, UK; 35, MWG-Biotech, Anzinger Strasse 7a, Ebersberg 85560, Germany; 36, Center for Comparative Genomics and Bioinformatics, Huck Institutes of Life Sciences, Departments of Biology, Statistics, Biochemistry and Molecular Biology, Computer Science and Engineering, and Health Evaluation Sciences, The Pennsylvania State University, University Park, Pennsylvania 16802, USA; 37, Roche Genetics and Roche Center for Medical Genomics, F. Hoffmann-La Roche Ltd, 4070 Basel, Switzerland; 38, Sanger Institute, The Wellcome Trust Genome Campus, Hinxton, Cambridgeshire CB10 1SA, UK; 39, Departments of Pathology and Genetics, Stanford University, Stanford, California 94305, USA; 40, S256 James H. Clark Center, Department of Computer Science, Stanford University, Stanford, California 94305, USA; 41, Department of Ecology and Evolutionary Biology, University of Arizona, Tucson, Arizona 85721, USA; 42, Centre de Recherches Mathématiques, Université de Montréal, 2920 Chemin de la tour, Montréal, Quebec H3T 1J8, Canada 〈http://www.crm.umontreal.ca〉; 43, Departamento de Bioquimica y Biologia Molecular, Instituto Universitario de Oncologia, Universidad de Oviedo, 33006 Oviedo, Spain 〈http://web.uniovi.es/degradome〉; 44, Department of Electrical Engineering and Computer Science, University of California Berkeley, Berkeley, California 94720, USA; 45, Department of Mathematics, University of California Berkeley, Berkeley, California 94720, USA; 46, Bioengineering Graduate Group, University of California Berkeley, Berkeley, California 94720, USA; 47, University of California, San Diego, Department of Mathematics, 9500 Gilman Drive, San Diego, California 92093-0112, USA 〈http://www-cse.ucsd.edu/groups/bioinformatics ; 48, University of California, San Diego, Department of Computer Science and Engineering, 9500 Gilman Drive, San Diego, California 92093-0114, USA 〈http://www-cse.ucsd.edu/groups/bioinformatics〉; 49, Howard Hughes Medical Institute, Center for Biomolecular Science \& Engineering, Mailstop SOE, Baskin School of Engineering, University of California, Santa Cruz, 1156 High Street, Santa Cruz, California 95064, USA; 50, UCSC Genome Bioinformatics Group, Center for Biomolecular Science and Engineering, Mailstop SOE, Baskin School of Engineering, University of California, Santa Cruz, 1156 High Street, Santa Cruz, California 95064, USA; 51, Institute of Medical Genetics, University of Wales College of Medicine, Heath Park, Cardiff, CF14 4XN, UK; 52, Department of Computer Science, University of Western Ontario, London, Ontario N6A 5B7, Canada; 53, Laboratory for Computational Genomics, Campus Box 1045, Washington University, St Louis, Missouri 63130, USA 〈http://genes.cse.wustl.edu〉; 54, Wellcome Trust Centre for Human Genetics, University of Oxford, Roosevelt Drive, Oxford OX3 7BN, UK; 55, The Wistar Institute, 3601 Spruce Street, Philadelphia, Pennsyvania 19104, USA; 56, US National Institutes of Health, National Human Genome Research Institute, 31 Center Drive, Bethesda, Maryland 20892, USA; 57, US National Institutes of Health, National Heart, Lung, and Blood Institute, Bethesda, Maryland 20892, USA; 58, Program in Structural and Computational Biology and Molecular Biophysics, Baylor College of Medicine, One Baylor Plaza, Houston, Texas 77030, USA 